\title{
Clogging of Microchannels by \\ Nano-particles due to Hetero-coagulation in Elongational Flow
}

\author{
zur Erlangung des akademischen Grades eines \\ DOKTORS DER INGENIEURWISSENSCHAFTEN (Dr.-Ing.) \\ der Fakultät für Chemieingenieurwesen und Verfahrenstechnik des \\ Karlsruher Institut für Technologie (KIT)
}

\author{
genehmigte \\ DISSERTATION
}

von

Msc. Kristina Georgieva

aus Nessebar, Bulgarien

Referent: Prof. Dr. Norbert Willenbacher

Korreferent: Prof. Dr.-Ing. habil. Hermann Nirschl

Tag der mündlichen Prüfung: 18.01.2013 
To Plamen 


\section{Acknowledgements}

I would like to express my sincere thanks to Prof. Dr. Norbert Willenbacher for his care and concern, inspiring guidance, encouragement, scientific support and enthusiastic supervision throughout this work. I am more grateful that I can adequately express.

I express my deep sense of gratitude to Prof. Dr. Hans Buggisch for the constructive comments, helpful discussions and genuine interest in the progress of my work. The theoretical model on collision frequency and interparticle contact time in flowing suspensions, developed by him and reported in this work, is highly beneficial and I appreciate his contribution to the success of this project.

I wish to express thanks to Dr.-Ing. Bernhard Hochstein for his support and encouragement during the course of this work

I am indebted to Dietmar Paul for his technical assistance in the development and construction of the ring-slit device. I am grateful for his friendly support, encouragement, helpful tips and for being so patient with my poor German at the begging of my $\mathrm{PhD}$ work.

I am sincerely grateful to Dr. Dick Dijkstra for the wholehearted cooperation, encouragement and concern.

I am thankful to Dr. Holger Fricke for providing the computational simulations reported in this work.

Special thanks must be given to my students Sarah Strubel, Andreas Waldecker and Martina Karner for their sincere assistance in pursuing my studies.

I thank all the colleagues in Prof Willenbacher's group for the support and warm atmosphere making a pleasure to work at the institute. Many thanks to Beate Oremek for the kind support throughout this work and to Erin Koos for critical reading of the manuscript.

I also gratefully acknowledge the financial support from Bayer MaterialScience AG.

Finally, I thank you the most, Plamen, for the love and understanding that make you the most important person in my life. I am truly grateful for the scientific support, helpful discussions and suggestions during the period of my research work. 


\section{CONTENT}

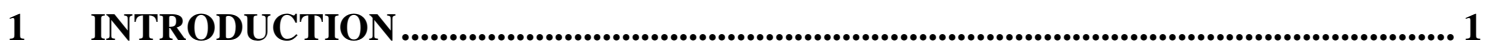

2 FUNDAMENTALS AND THEORETICAL BACKGROUND................................... 3

2.1 Properties of charged interfaces..................................................................................... 3

2.1.1 Electrostatic double layer - origin and structure …............................................... 3

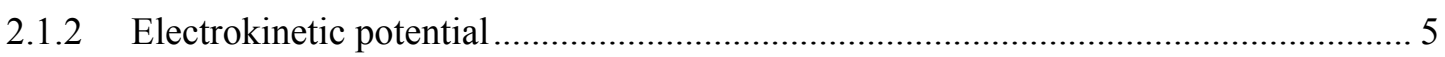

2.2 Interparticle interactions in dispersions............................................................... 7

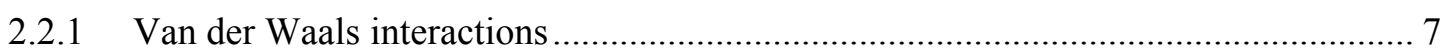

2.2.2 Interactions between spherical double layers ..................................................... 8

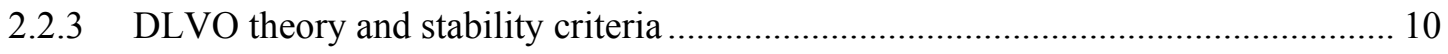

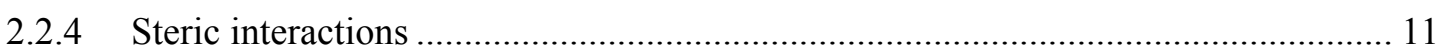

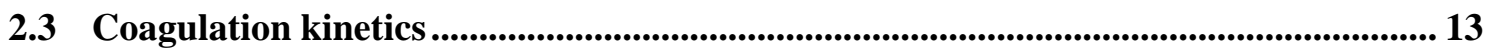

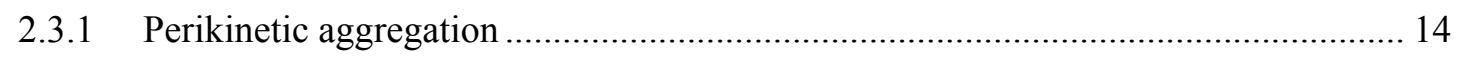

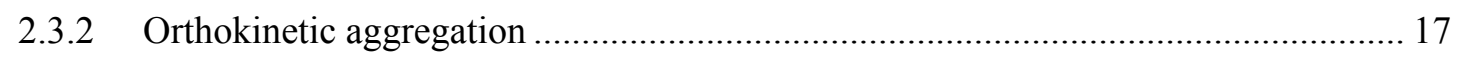

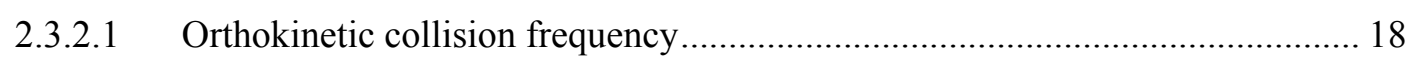

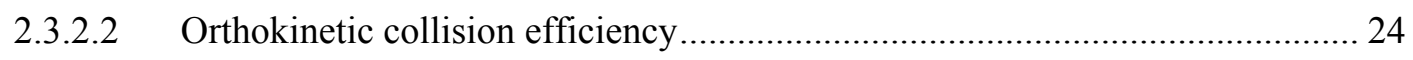

2.4 Criteria for stability of flowing colloid suspensions ..................................................... 31

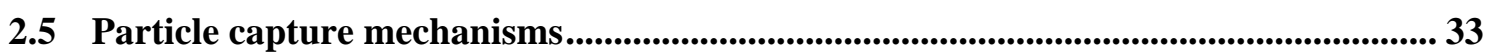

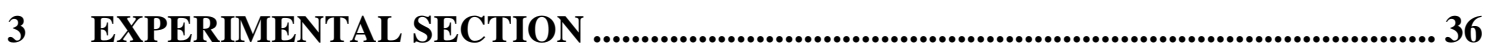

3.1 Experimental apparatus and procedure …...................................................................... 36

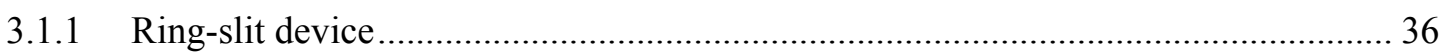

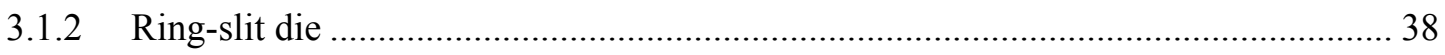

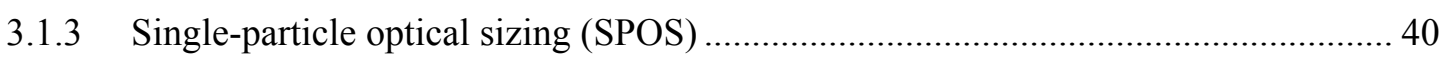

3.1.3.1 Description of the SPOS technique and procedure …..................................... 41

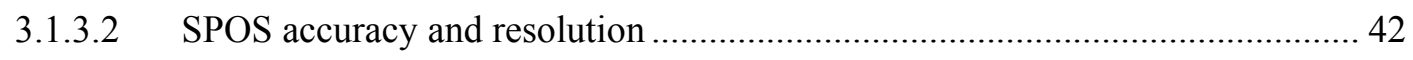

3.1.3.2.1 Maximum coincidence and sizing accuracy .............................................. 42

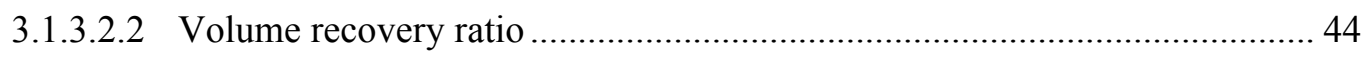

3.1.3.2.3 Analysis of micron-sized particles in nanodispersions ................................. 45

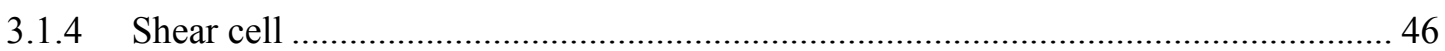

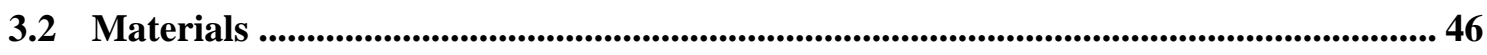

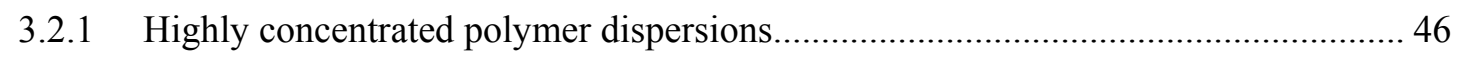

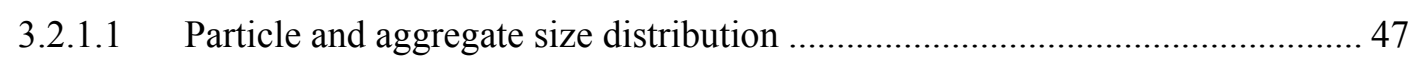

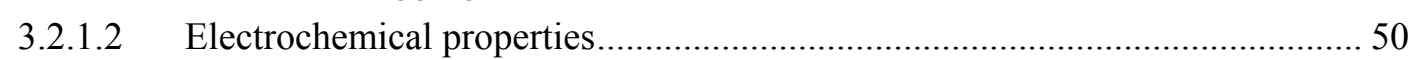


3.2.1.2.1 Procedure for preparing dispersions of known ionic strength

3.2.1.3 Identification of the stabilization mechanism with high frequency rheology

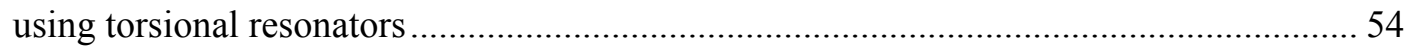

3.2.1.4 Rheological estimation of electrostatic double-layer thickness ........................ 55

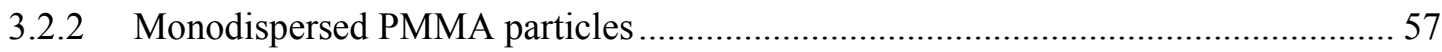

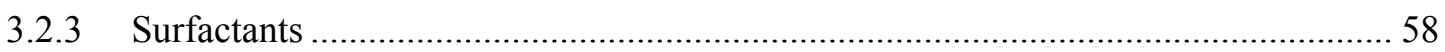

$4 \quad$ EXPERIMENTAL RESULTS AND DISCUSSION ..................................................... 59

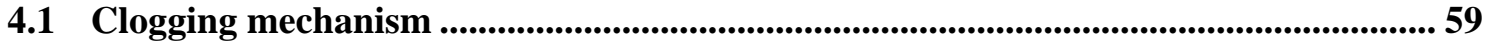

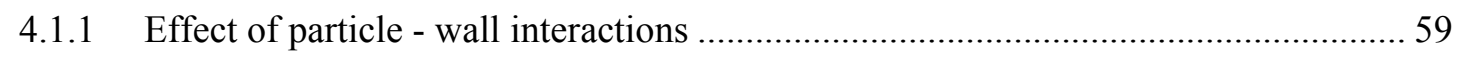

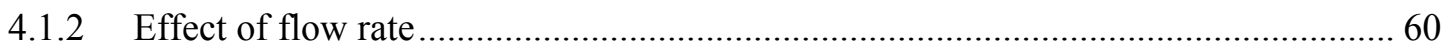

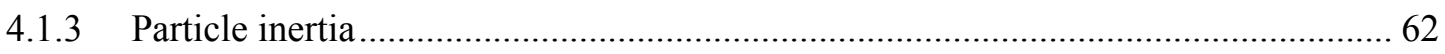

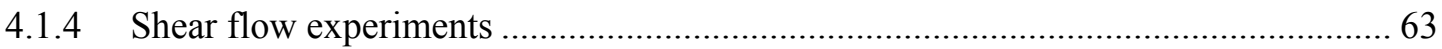

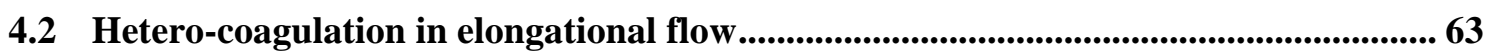

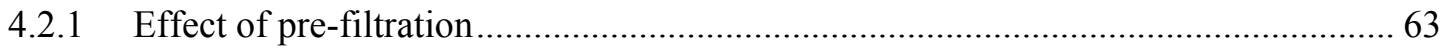

4.2.2 Hetero-coagulation with monodispersed PMMA particles........................................... 64

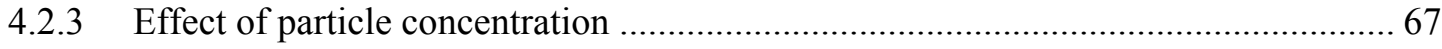

4.3 Impact of colloidal properties on flow induced aggregation ........................................... 68

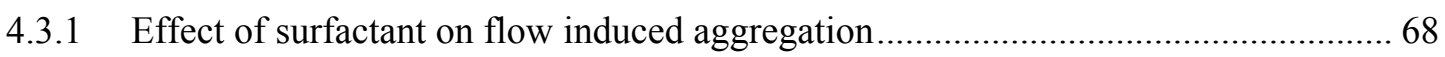

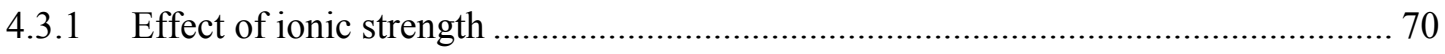

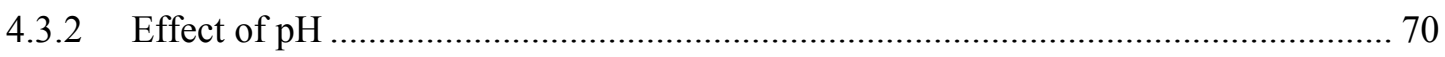

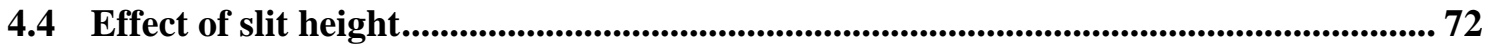

4.5 Flow kinematics parameter and their influence on flow-induced aggregation........... 74

4.5.1 Interpretation of the effect of flow rate on flow induced aggregation...................... 74

4.5.2 Effect of entrance angle on flow induced aggregation ............................................. 77

4.6 Influence of particle properties on the pressure increase .............................................. 79

$5 \quad$ CONCLUSION AND FUTURE PROSPECTS ............................................................... 82

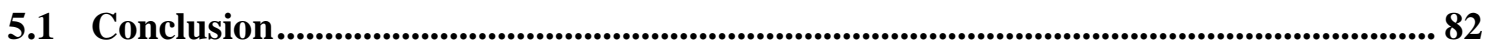

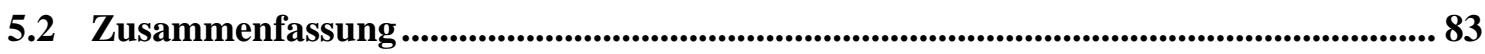

5.3 Future prospects .................................................................................................................... 86

6 APPENDIX

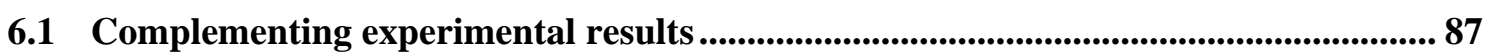

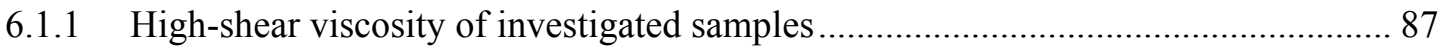

6.1.2 Examination of the sensor calibration for the AccuSizer 780 AD........................... 88 


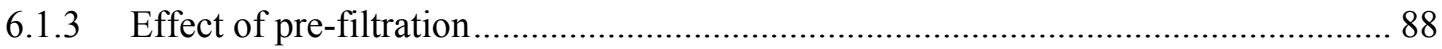

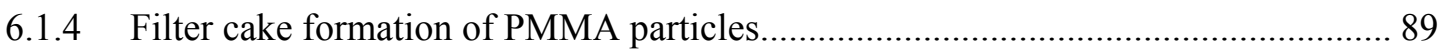

6.1.5 Effect of surfactant on flow induced aggregation................................................. 90

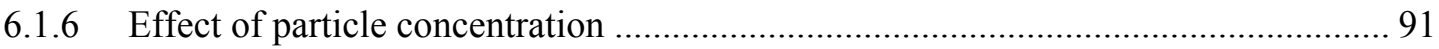

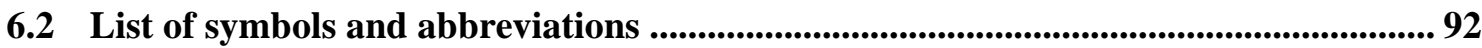

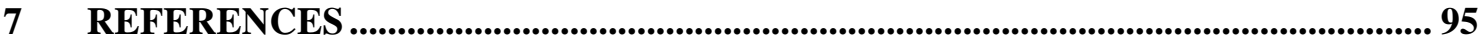




\section{Introduction}

Nano-particle suspensions are encountered in variety of industrial processes and have an outstanding importance to our everyday life since a lot of products like paints, coatings, inks, pharmaceutical and cosmetics formulations, as well as various food products are formulated as suspensions of fine particles dispersed in a liquid phase. To guarantee product quality and performance it is decisive to maintain dispersion stability over months or even years. Dispersion stability is especially critical during manufacturing, processing and application, when they are exposed to complex flow fields including strong extensional components (convering streamlines). Te phenomenon of flowinduced aggregation may occur even for dispersions with repulsive particle interactions providing sufficient shelf-life at rest. It is relevant not only for classical technical operations like coating, pumping or filtration, but also for the application of concentrated suspension in upcoming processing technologies based on microfluidic devices.

Although much is known about colloidal stability at rest or in shear flow, little attention has been paid to the process of agglomeration in nano-particle dispersions with industrially relevant particle concentration (volume fraction $>30 \%$ ) exposed to converging flow fields. Converging flow occurs whenever liquids are forced to flow from large reservoirs through an orifice or sudden contraction. Formation of aggregates in this critical flow region can cause blockage at the inlet of microchannels or nozzles and thus compromise the efficiency and reliable operation of processing technologies such as ink-jet printing, industrial high speed coating processes or any technical operation or microfluidic system where strong flow contraction occurs.

In order to study this phenomenon experimentally we use a ring-slit device, which has already been established to characterize flow-induced aggregation for industrial quality control and product development purposes [1]. This device embodies a capillary viscometer and a ring-slit die. The ring-slit geometry allows us to maintain a small gap height $(10-30 \mu \mathrm{m})$, corresponding to a high contraction ratio (1:1000), and a large crosssectional area at the same time. When aggregation takes place the slit entrance is gradually clogged resulting in a strong pressure increase. Thus aggregates are formed and detected simultaneously. This method allows for a variation of flow conditions over a wide range. By variation of the volumetric flow rate, slit height and slit entrance angle we are able to control the collision frequency, contact time of colliding particles and the total deformation of a fluid element as well as velocity gradient in the entrance region where aggregation takes place. 
The aim of the research described in this thesis was to investigate the clogging of a microchannel by concentrated nano-particle suspensions in order to get a fundamental understanding of the physical mechanisms that lead to flow-induced aggregation and correspondingly to blockage of narrow slits. We focus on dispersions with repulsive particle interactions guaranteeing high stability at rest (shelf life of more than 6 month). Our experiments exclude, that channel clogging is due to retention of pre-existing aggregates, fouling or hydrodynamic bridging. Aggregation is also proved not to occur in the pure shear flow within the straight narrow slit. Instead, we demonstrate that clogging of the microchannel is induced by hetero-coagulation between primary colloidal particles and micron-sized impurities present at concentrations on the order of $100-1000 \mathrm{ppm}$. It will also be shown that the flow-induced aggregation can be controlled by adjusting colloidal particle interactions.

Further, this phenomenon depends strongly on the flow rate and the slit clogging is faster the lower the flow rate is. The effect of flow rate is explained with the dynamic balance between aggregation and breakup of aggregates under high shear conditions. Considering an appropriately expressed Breakage number and the aggregation probability based on the interparticle contact time, we will demonstrate that the aggregation efficiency decreases with increasing flow rate, which limits the maximum aggregate size and thus explains the observed flow rate dependence of the slit clogging phenomenon. Furthermore, it will be shown experimentally that smaller slit entrance angles promote the flow-induced aggregation in the ring-slit device. These results will be interpreted with the help of computational fluid dynamic (CFD) simulations, considering the residence time of the particles in the flow field at the slit entrance.

The findings of this study suggest the following strategies to prevent clogging of microchannels by flowing nanodispersions:

- Reduction of the amount and size of micron-sized impurities

- Improving colloidal stability of the primary nano-particles

- High flow rates, leading to reduction of the interparticle contact time and breakup of aggregates under the high shear conditions

- Steep entrance angles in order to decrease the particle residence time in the converging flow field 


\section{Fundamentals and theoretical background}

The objective of this chapter is to give a short overview of the general background to the research discussed in this thesis. The first four sections review the state of research relevant to aggregation of flowing suspensions. Theoretical examinations of colloidal interactions of suspended particles are presented and discussed in section 2.1 and section 2.2. Then a detailed overview of aggregation kinetics is given in section 2.3. Criteria for mechanical stability under shear are discussed in section 2.4. Since this work concerns the flow-induced aggregation, which causes the clogging of microchannels, the possible particle capture mechanisms will be also considered and briefly discussed (section 2.5)

\subsection{Properties of charged interfaces}

Particles dispersed in water can obtain surface charge for different reasons, which will be briefly considered in section 2.1.1. The distribution of ions around a charged interface in an electrolyte solution gives rise of an electrical double layer. The most important properties and physical models to describe the electrical double layer will also be discussed in section 2.1.1. Particle electrophoresis as an experimental approach to determine the electrical potential at the plane of shear will be considered and the most common expressions to relate the electrophoretic mobility to the electrokinetic potential will be discussed in section 2.1.2.

\subsubsection{Electrostatic double layer - origin and structure}

Particles dispersed in aqueous media acquire a surface electric charge for various reasons. The most common reasons are the ionization of surface groups in the presence of water ( $\mathrm{pH}$ dependent), ion adsorption (such as surfactant ions) and ion dissolution (such as in the case of salt type minerals). In an electrolyte solution, the charge on a particle surface is balanced by a corresponding number of counter-ions in the dispersion medium. The question remains how counter-ions are distributed in the solution around the particles. This can be understood by considering the effect of electrostatic and diffusion forces, the electrostatic force tends to attract counter-ions to the particle surface, whereas the thermal motion causes the counter-ions to be spread out in the solution. This leads to the formation of an electric double layer that consists of two regions: a region in the immediate vicinity of the charged surface and diffuse region in which the charge density varies with distance from interface. According to the Gouy-Chapman model [2], the potential in the diffuse double layer, in the case of infinite flat surface, is represented at any point by the relation: 


$$
\Psi=\Psi_{0} \exp (-\kappa x)
$$

where $\Psi_{0}$, is the surface potential, $\kappa$ is the Debye-Hückel parameter and $\mathrm{x}$ is the distance of the charged surface. The Gouy-Chapman approach is based on the DebyeHückel approximation, assuming a low surface potential. Since at distance $x=\kappa^{-1}$ the potential in the diffuse layer is reduced to $\Psi_{0} \mathrm{e}^{-1}$, the length $\kappa^{-1}$ is usually known as the thickness of the diffuse layer. For the general case of electrolyte solutions containing different dissolved salts, $\kappa^{-1}$ is defined by:

$$
\kappa^{-1}=\sqrt{\frac{\varepsilon_{r} \varepsilon_{0} k_{B} T}{e^{2} \sum n_{i} z_{i}^{2}}}
$$

where $\varepsilon_{\mathrm{r}}$ is the relative dielectric constant of the dispersion medium, $\varepsilon_{0}$ the permittivity of vacuum, $\mathrm{k}_{\mathrm{B}} \mathrm{T}$ is the thermal energy, $e$ is the elementary unit of charge, $z_{i}$ is the valence of the ions of type $i$, and $n_{i}$ is the number concentration of the $i$ ions. The $\kappa^{-1}$, known as Debye length is highly relevant to the dispersion stability. It can be seen from equation (2) that, as the ion concentration and valence increases the Debye length decreases, which is referred to as double layer compression. However, highly charged ions will be more effective than monovalent ions due to the strong effect of ion valence. In aqueous dispersions $\varepsilon_{r} \varepsilon_{0} k \mathrm{~T}$ is almost constant over a broad temperature range and $\kappa^{-1}$ varies only slowly with the temperature.

The main limitation of the Gouy-Chapman model is the assumption of ions as point charges. Taking into account that real ions have a significant size, especially if they are hydrated we recognize that this limits the distance of closest approach to a charge interface. This concept is originally due to Stern [3] and the distance $(\delta)$ which defines the region inaccessible to the counter-ions, is usually known as Stern layer. The Stern layer contains a certain fraction of the counter-ions, and the remaining counter-ions are distributed within the diffuse layer. Figure 1 shows a schematic illustration of the variation of electrical potential from the surface according to the Stern-Gouy-Chapman model of the electrical double layer at a flat interface. Across the Stern layer the potential falls linearly from the surface potential $\Psi_{0}$ to a value $\Psi_{\delta}$ known as Stern potential. From the boundary of the Stern layer into the solution the potential varies with the distance according to the Debye-Hückel approximation if $\Psi_{\delta}$ is rather low.

So far, flat charged plates have been concerned but these are not appropriate models for the spherical particles important for this thesis. For the case of low $\Psi_{\delta}$, the variation of potential with radial distance $r$ from the centre of the spherical particle with radius $a$ is given by the following: 


$$
\Psi=\Psi_{\delta} \frac{a}{r} \exp [-\kappa(r-a)]
$$

In the case of Stern (surface) potentials of several tens of millivolts the Debye-Hückel approximation is not appropriate and equation (3) becomes incorrect. However, numerical approaches of the problem allow an exact computation of the potential of electrical double layers without using the linear approximation [5].

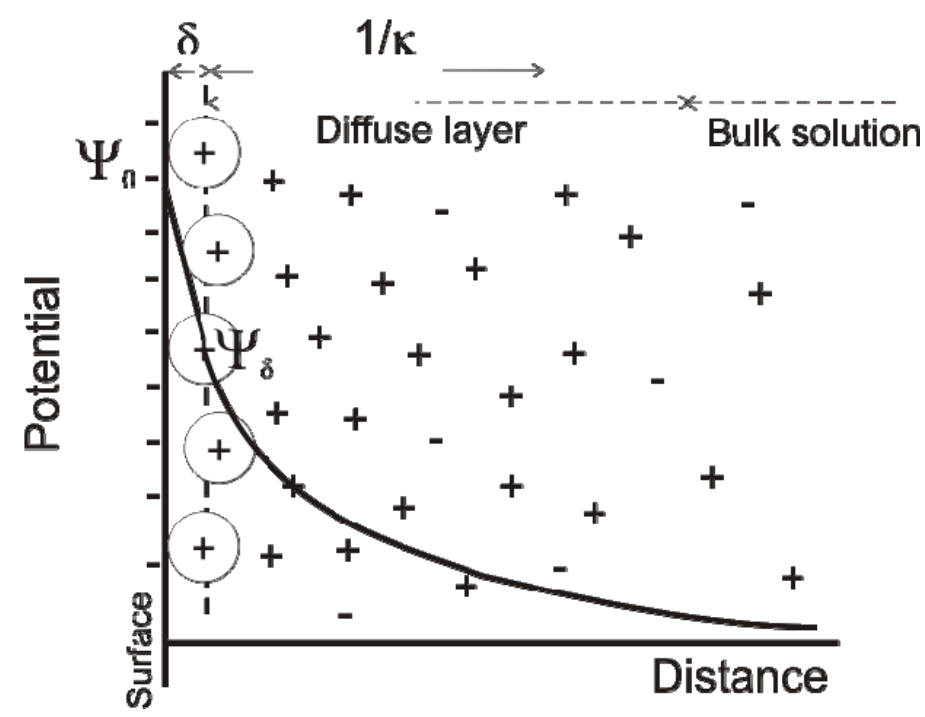

Figure 1. Schematic representation of the electrical double layer adjacent to a negatively charged surface according to the Stern-Gouy-Chapman model [4].

\subsubsection{Electrokinetic potential}

The most widely used experimental approach to estimate the Stern potential is through the use of electrokinetic techniques. The electrokinetic phenomena depend on the potential at the plane of shear, which separates the effectively bounded to the charged surface counter-ions and the counter-ions in the diffuse layer that are free to move with the liquid. The plane of shear is assumed to be located close to, perhaps just outside the Stern plane since the ions are hydrated and certain amount of solvent will probably move with the charged surface [6]. The potential at the shear plane is called electrokinetic or zetapotential ( $\zeta$-potential) and is assumed to be approximately equal to $\Psi_{\delta}$. Although this assumption is not strictly justified, there is a clear correlation between colloidal stability and zeta-potential. Colloidal particles are found to be stable when the zeta-potential exceeds a certain threshold value [7].

There are number of techniques to determine the $\zeta$-potential, most of which are based on the motion of particles in an electric field [2] Particle mobility in this case is known as electrophoretic mobility and is defined as the electrophoretic velocity, i.e. the veloci- 
ty of the particles relative to stationary liquid, divided by the electric field strength. The electrophoretic motion of particles can be detected by various techniques; the one used in this study is laser-doppler velocimetry (Zetasizer Nano, Malvern). Two crossed laser beams generate a set of interference fringes and particles moving through these fringes scatter light from the region of interference into a photodetector. The light scattered by moving particles shows the Doppler shift. Thereby the frequency of the fluctuating light intensity is related to the particle velocity [4][6].

The zeta-potential, $\zeta$, can be derived from the determined electrophoretic mobility, U, by application of Henry equation [9]:

$$
U=\frac{2 \varepsilon \zeta}{3 \eta} f(\kappa a)
$$

where $\varepsilon$ and $\eta$ are the values of the permittivity and viscosity respectively and $f(\kappa a)$ varies between 1 for small $\kappa a$ and $3 / 2$ for large values of $\kappa a$. This equation is based on the assumptions that the external filed and the field of the double layer are additive, the potential is low enough to justify the Debye-Hückel approximation and the permittivity and viscosity are constant throughout the diffuse double layer.

For small spherical particles $\left(\mathrm{a}<10^{-8} \mathrm{~m}\right)$ and low ionic strength of the surrounding liquid $\left(10^{-5} \mathrm{M}\right.$ for $1: 1$ electrolyte), so that the diffuse layer is of greater extent than the particle radius (small $\mathrm{\kappa a}$ ) the Henry equation reduces to the Hückel equation:

$$
U=\frac{2 \varepsilon \zeta}{3 \eta}
$$

This equation is not applicable for the most particles dispersed in water. It is more suited to elecrtrophoresis of macromolecules.

For particles large compared to Debye length ( $\mathrm{\kappa a}>>1$ ), the electrophoretic mobility is related to $\zeta$-potential by the Smoluchowski equation:

$$
U=\frac{\varepsilon \zeta}{\eta}
$$

This expression is useful for determining zeta-potential of fairly large particles in electrolyte solutions with high ionic strength.

In practical cases we often deal with particles of colloidal size dispersed in electrolyte solution of moderate ionic strength, for which neither Smoluchowski nor Hückel equation is appropriate. In all this cases the Henry's equation [9] should be applied taking into account the double layer relaxation effect, which arises from the distortion of the diffuse layer during the motion of charged particle. As the particle ion atmosphere can 
not respond immediately to the particle motion, it lags behind the particle. The resulting asymmetrical charge distribution exerts an additional retarding force and cause particles to move more slowly. Relaxation can be neglected in the case of very small or large кa, but it is significant for intermediate values, particularly at high potentials [10]. Wiersema et al. [11] as well as $\mathrm{O}^{\prime}$ Brien and White [12] developed models to account for high surface potentials and the double layer relaxation effect. For кa values between 0.1 and 100 a very complex relation between electrophoretic mobility and $\zeta$-potential has been found.

\subsection{Interparticle interactions in dispersions}

The phenomenological description of dispersion stability depends on understanding the nature of the forces of attraction and repulsion between particles. These colloidal forces comprise several components, van der Waals, electrical double layer and steric forces, which act over short distances and are dependent on particle surface properties. The purpose of the present section is to outline the basic ideas and equations describing these interactions in order to illustrate how they affect colloidal stability.

\subsubsection{Van der Waals interactions}

All solid particles attract each other through the van der Waals forces due to spontaneous electron fluctuation at the atomic level. The attraction results from the electrostatic interactions among dipoles, which can be categorized as follow: Keesom interactions (permanent dipole-permanent dipole interaction), Debye interactions (permanent dipole -induced dipole interactions), and London or dispersion interactions (induced dipoleinduced dipole interactions). The later are the major component of van der Waals forces and the most important from colloidal stability point of view.

According to the classical theoretical approach, the evaluation of London-van der Waals attraction between two macroscopic bodies, of known geometry, can be approximated by adding up all the relevant intermolecular interactions. This method is due largely to Hamaker [13] and can be easily applied to different geometries. For two spheres of radius $a_{1}$ and $a_{2}$, at center-to-center separation $r$, the van der Waals energy of attraction per unit area $\Psi_{\mathrm{vdW}}$ is found to be the following [14]:

$$
\Psi_{v d W}=-\frac{1}{6} A_{H}\left(\frac{2 a_{1} a_{2}}{r^{2}-\left(a_{1}+a_{2}\right)^{2}}+\frac{2 a_{1} a_{2}}{r^{2}-\left(a_{1}-a_{2}\right)^{2}}+\ln \frac{r^{2}-\left(a_{1}+a_{2}\right)^{2}}{r^{2}-\left(a_{1}-a_{2}\right)^{2}}\right)
$$

where $A_{H}$ is the Hamaker constant, which is a material property that represents the strength of the van der Waals attraction between macroscopic bodies. Typically the Hamaker constants varies between $10^{-19}$ and $10^{-21} \mathrm{~J}$ at room temperature [15]. 
For spheres of equal radius $a$ and interparticle separation $h<<2 a$ equation (7) simplifies to:

$$
\Psi_{v d W}=-\frac{A_{H} a}{12 h}
$$

This approximation applies only at close approach and is quite inaccurate for submicron particles but it provides a good analytical estimation that is acceptable for most practical purposes. The most important features of this expression are the inverse dependence of the interaction energy on the separation distance and the direct dependence on particle size.

It should be noted that the values of $\Psi_{\mathrm{vdW}}$ from the expressions above will be overestimated at large distances, since a finite time is needed for propagation of the interaction and the correlation between temporary induced dipoles in the interacting bodies becomes smaller and leads to reduced attraction. Therefore corrections should be carried out in order to account for the retardation effects [16].

\subsubsection{Interactions between spherical double layers}

Another very important type of interparticle interaction is the electrical double layer interaction, which arises when the diffuse double layers of charged particles in electrolyte solutions start to overlap.

The usual theoretical treatment of double layer interactions involves calculations at constant surface charge density and constant surface potential. These two well defined situations, recognized by [17] are assumed to be two hypothetical extremes and the true situation lie, in general, somewhere between constant potential and constant charge.

A simple expression for the interaction energy between charged flat plates in an electrolyte solution is obtained [18] based on the assumptions that the potential in the region of the minimum (at the midpoint position between two overlapping double layers) is sufficiently small and is just the sum of the contributions from the isolated plates. This linear superposition approximation leads to the following expression for the potential energy of interaction [14]:

$$
\Psi_{e l}=64 k_{B} T n_{\infty} \kappa^{-1} \gamma_{1} \gamma_{2} \exp (-\kappa h)
$$

where $\mathrm{n}_{\infty}$ is the bulk number density of ions, $\kappa$ is the Debye-Hückel parameter defined in equation (2) and $\gamma$ represents the reduced surface potential $\left(=z e \Psi_{0} / k_{B} T\right)$. It should be noted that equation (9) is derived assuming that the dispersion medium contains only symmetrical electrolytes and that the Debye-Hückel approximation holds, i.e $\Psi_{0}$ is small $(<25 \mathrm{mV})$. Furthermore the validity of equation (9) requires that the plate separation is large compared with the Debye length ( $\kappa h>>1)$. 
The potential energy of interactions between two spherical particles can be obtained via the Derjaquin approximation [19], which is based on the corresponding plate-plate expression. The basic idea of the Derjaguin approximation is that the surface of sphere may be assumed to be made up of as series of infinitely thin parallel ring-shaped flat plates. The Derjaguin approximation may be applied only when the particle radius is larger than the thickness of the double layer $(\kappa a>>1)$ and the interparticle separation, $h$, is much smaller than the particles size $(h<<a)$.

When the Derjaguin approximation is applied to the interaction of two identical spheres with low surface potential, the resulted expression is referred to as Hogg-HealyFuersteneau formula [20]:

$$
\Psi_{e l}=\frac{\varepsilon a \psi_{0}^{2}}{2} \ln [1+\exp (-\kappa h)]
$$

This equation is identical to that derived by Derjaguin and is accurate for small $\kappa h$ and large $\kappa a$ as discussed above.

Sader et al. modified the Hogg-Healy-Fuersteneau expression so that it is valid even for modest $\kappa a$ values and for $\kappa h$ in the whole range of practical interest [21]:

$$
\Psi_{e l}=\frac{4 \pi \varepsilon a \psi_{0}^{2}}{l} \ln [1+\exp (-\kappa a(l-2))]
$$

where $l$ is the dimensionless interparticle separation defined as the ratio between center-to-center separation (r) and particle radius (a).

All theoretical treatments lead in general to the following conclusions:

- interaction potential between spherical particles depends exponentially on the distance between them.

- approaching particles start to influence each other at large distance of separation and the separation at which the repulsion start to be significant is determined by the Debye length $\kappa^{-1}$. Hence $\kappa^{-1}$ determines the range of repulsive interactions. For instance at high ionic strength $\left(\right.$ small $\left.^{-1}\right)$ the interaction is of short range and decays rapidly with the distance; at low ionic strength the interaction is correspondingly of longer range.

- the electrostatic repulsion is directly proportional to the particle size as in the case of van der Waals attraction (equation (8)). 


\subsubsection{DLVO theory and stability criteria}

The theory of electric double layer interactions combined with van der Waals attraction and the influence of these interactions on colloidal stability was independently developed by Derjaguin and Landau [22] and by Verwey and Overbeek [17] and therefore called DLVO-theory.

Basically, the DLVO theory involves estimation of the total interaction energy in terms of interparticle distance, assuming linear superposition of van der Waals attraction and delectrical double layer repulsion. Thus the total interaction potential can be written as:

$$
\Psi(h)=\Psi_{v d W}(h)+\Psi_{e l}(h)
$$

The general character of the resulting interaction potential versus distance curves depends on the magnitude and the distance dependence of the attractive and the repulsive terms. Figure 2 demonstrates a typical potential energy diagram for interaction of electrostatically stabilized particles. The most important feature of this diagram is the potential energy barrier $\left(\Psi_{\max }\right)$, which is in this case much higher than the average thermal energy of particles and the dispersion is kinetically stabilized. When $\Psi_{\max }=0$, coagulation becomes a rapid diffusion controlled process. Thus potential energies are often expressed in $k_{B} T$ units. The height of the energy barrier strongly depends on the magnitude of $\Psi_{\delta}$ (or $\zeta$ ) and on the Debye length $\left(\kappa^{-1}\right)$. At low ionic strength, $\kappa^{-1}$ is large and a substantial repulsive energy barrier exists, whereas at high ionic strength the Debye length is short and the barrier does not exist or it is very small.

Another characteristic feature of the potential energy curve is the secondary minimum, which arise when the van der Waals attraction is greater than the electrostatic repulsion at sufficiently large separation distance. The secondary minimum is important only when it is moderately deep $\left(>5 k_{B} \mathrm{~T}\right)$ and when the potential energy barrier is so large that the particles do not pass over it into the primary minimum. These conditions are met only with large particles (micron-sized particles) and at moderately high ionic strength, so that the range of repulsive interactions is reduced and particles can come close together, where the van der Waals forces are greater. For nano-particles the secondary minimum is typically shallow and never deep enough for flocculation. However, secondary minimum flocculation gives rise to fairly week aggregates and a shear force can easily separate the particles again. 


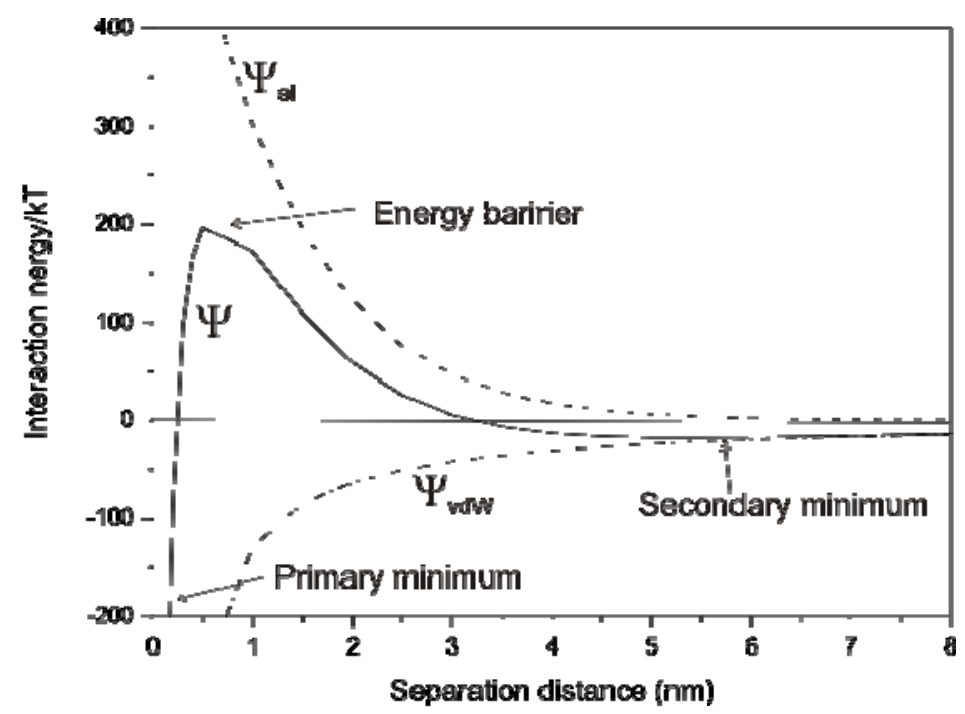

Figure 2. Interaction potential for spherical particles with a diameter of $1 \mu \mathrm{m}$ in a $50 \mathrm{mM}$ solution of 1:1 electrolyte ( $\zeta=30 \mathrm{mV}, A_{H}=1.3 \times 10^{-20} \mathrm{~J}$ ). The curves show the electrical $\left(\Psi_{e l}\right)$ van der Waals ( $\left.\Psi_{v d W}\right)$, and total $(\Psi)$ interaction energy [4].

The infinitely deep primary minimum in the DLVO representation of the interaction potential (equation (12)) is not consistent with studies of agglomerate breakage and reversible agglomeration. Thus the DLVO theory often needs to be modified to account for the short range repulsive forces between surface atoms as their atomic electron clouds overlap. This is the so called Born repulsion, which keeps the particles from penetrating each other. A realistic expression of the Born potential $\left(\Psi_{\text {Born }}\right)$ between identical spheres has been derived by Feke et al [23]:

$$
\Psi_{\text {Born }}=-\frac{N_{12} A_{H}}{l}\left[\frac{l^{2}-14 l+54}{(l-2)^{7}}+\frac{-2 l^{2}+60}{l^{7}}+\frac{l^{2}+14 l+54}{(l+2)^{7}}\right]
$$

where $\mathrm{N}_{12}$ is a parameter expressing the strength of the Born repulsion relative to van der Waals attraction. Typically, $\mathrm{N}_{12}$ is in the range of $10^{-18}$ to $10^{-23}$ for most colloidal systems. Including the Born potential into the interparticle potential (equation (12)) restrict the primary minimum to finite depth. Feke et al. pointed out that in the case of strong electrostatic repulsion the Born forces may even eliminate the primary minimum. However, the Born interaction may not be of great importance in aqueous dispersions because of other short range effects, such as those caused by hydrated ions, which may prevent particles from coming in physical contact thus making the primary minimum of finite depth [7].

\subsubsection{Steric interactions}

Stability of dispersions is often higher than expected on the basis of the DLVO theory. The adsorption of polymers on the particle surface can give rise to another stabilizing 
mechanism, which is usually known as steric stabilization [24]. The role of polymers on colloidal stability is very complicated. At low concentrations of long chain polymers with high affinity to particle surface, the polymer chains may adsorb onto more than one particle causing bridging flocculation. On the other hand, in the case of non-adsorbing polymers, they will be free to move in the dispersion medium and can cause depletion flocculation. This phenomenon may be understood by considering two particles approaching one another in a solution of free polymer. As the gap between them becomes too small to contain the polymer molecules, the local osmotic conditions due to the polymer concentration deference will cause the pure dispersion medium to flow out from the gap between particles thus causing particles to flocculate [6].

The most effective stabilizers are block copolymers which have segments of the polymer chain that are designed to optimize the affinity for the surface as well as segments that interact preferentially with the dispersion medium [6]. The extent of extension of the polymer chains into the medium depends on how they interact with the medium.

When two particles approach sufficiently close, their adsorbed layers may interpenetrate and, depending on the affinity of polymer segments to each other and the affinity to solvent molecules (polymer-polymer and polymer-solvent interactions), repulsion or attraction can result. If the dispersion medium is a good solvent for the adsorbed polymer, it expands away from the surface and adsorbed layer interpenetration will result in interparticle repulsion but if the dispersion medium is a poor solvent the polymer avoid the contact with the solvent and attraction between interpenetrating polymer chains is favored.

Let us consider the case of aqueous latex dispersions, the subject of this study. If the stabilizing copolymer contains hydrophilic and hydrophobic segments, it will attach strongly to the latex particle surface with the hydrophobic part and the hydrated polymer chains will expand into the solution to gain configurational enthropy. On collision between particles, the increased local density of polymer segments leads to a restriction in configurational freedom. The loss of entropy and, therefore gain in the free energy, results in repulsion between particles. Stabilization could also be a result of positive enthalpy, which reflects the release of water molecules from the hydrated polymer chains as they interpenetrate. As usual, the relationship between the change in enthalpy, $\Delta \mathrm{H}_{\mathrm{e}}$, entropy $\Delta \mathrm{S}$ and Gibbs free energy $\Delta \mathrm{G}$ is given by the following:

$$
\Delta G=\Delta H_{e}-T \Delta S
$$

As evident, stabilization will occur at any temperature when $\Delta \mathrm{H}_{\mathrm{e}}$ is positive and $\Delta \mathrm{S}$ is negative. If $\Delta \mathrm{H}_{\mathrm{e}}$ and $\Delta \mathrm{S}$ are both positive the enthalpic term is responsible for the stability and dispersion should become less stable with increasing temperature (enthalpic stabilization). Whereas if $\Delta \mathrm{H}_{\mathrm{e}}$ and $\Delta \mathrm{S}$ are negative, the entropic term prevents aggregation 
and the resistance to aggregation decreases as the temperature decreases. This stabilization mechanism is called entropic stabilization. It should be noted that, for a given polymer the stabilization mechanism may be enthalpic or entropic depending on the solvent. For aqueous dispersions the enthalpic stabilization tends to be the more common mechanism, correspondingly increasing temperature dispersion stability decreases [26] [6].

Quantitative understanding of polymer induced forces has been developed by considering various individual effects such as osmotic repulsion, entropic contribution, attraction or repulsion due to overlap of the tails of the adsorbed or grafted polymer layers, and so on. Several quantitative theories have been developed to express the interaction energy between two polymer coated particles as a function of surface to surface distance [27] [28] [29]. To a first approximation, the repulsion can be assumed to become infinite as the adsorbed layers begin to overlap and zero at greater separation [28]. Thus, aggregation in the deep primary minimum is virtually impossible by steric interactions and the aggregation is rather weak. The polymer induced forces are short-range and the stabilizing layer needs to be sufficiently thick to screen the van der Waals attraction and prevent particles from aggregating. As the van der Waals attraction energy is proportional to the particle size (equation (8)) larger particles will need thicker polymer layers to provide dispersion stability.

\subsection{Coagulation kinetics}

In the previous section, we have considered complete dispersion destabilization in the absence of a potential energy barrier, which means that particles adhere on contact and every collision results in attachment. This is known as "rapid" coagulation. However, even when there is an energy barrier, some collisions will result in coagulation because a certain fraction of these particles will have sufficient energy to overcome the barrier. The fraction of successful collisions is called the collision efficiency, which is equal to zero in the case of strong net repulsion and approaches unity when attraction forces dominate.

The mathematical treatment of aggregation has been devised by considering the process as two steps: transport of particles toward each other and attachment on contact. The transport step is achieved by Brownian motion of the particles, imposed velocity gradients or differences in the sedimentation velocity of individual particles. Attachment is dependent on short-range forces such as van der Waals attraction and electrostatic or steric repulsion. Depending on the transport mechanism, coagulation is known as perikinetic in the case of diffusion driven coagulation and orthokinetic for when velocity gradient is the dominant transport mechanism. Nearly all flocculation models are based on this fundamental treatment of the aggregation process in which the rate of ag- 
gregation is expressed as the product of collision frequency and collision efficiency. While the collision frequency is a function of the transport mechanism (perikinetic, orthokinetic or differential sedimentation), collision efficiency depends on the interparticle colloidal interactions and hydrodynamic or viscous interactions which tend to hinder the particles contact.

\subsubsection{Perikinetic aggregation}

Let us consider the case of dilute dispersions of identical spherical particles with motion governed by Brownian movement. The rate at which these particles coagulate depends on the collision frequency and the probability that their thermal energy is sufficient to overcome the repulsive potential energy barrier to aggregation.

The first major attempt at modeling the aggregation process was made in 1917 by Smoluchowski [30]. He formulated the equations for the rate of rapid coagulation assuming that every collision results in aggregation Another important assumption is that collisions involve only two particles, which allows treating aggregation as a second order process, in which the change of concentration of identical primary particles is proportional to the second power of their concentration.

$$
\frac{d n}{d t}=-k_{r} n^{2}
$$

where $n$ is the number density at time $t$ and $k_{r}$ is the second-order rate constant. The minus sign indicates the reduction of particles due to aggregation. This equation is appropriate only for the very early stages of aggregation of dilute monodisperse dispersions, since it considers only the loss of primary particles due to individual collisions. After a period of aggregation, the dispersion contains aggregates of different size at different concentrations. The following expression describes the rate of change of the concentration of aggregates of size $p$, taking into account the rate of formation of $p$-size aggregates from smaller species and the rate of vanishing of aggregates of size $p$ due to collision with any other aggregates:

$$
\frac{d n_{p}}{d t}=\frac{1}{2} \sum_{i<p} k_{i, p-i} n_{i} n_{p-i}-n_{p} \sum_{i} k_{i p} n_{i} \quad p=1 \ldots p_{\max }
$$

where $\mathrm{k}_{\mathrm{ip}-\mathrm{i}}$ and $\mathrm{k}_{\mathrm{ip}}$ are the correspondent rate constants. The summation in the first term in this equation means that we count each collision twice, therefore the factor of one half is introduced. Equation (16) is known as the population balance equation, since it describes the populations of particles of various sizes. Finding appropriate values for the collision rate constant is actually the main difficulty in applying equation (16). In general, it depends on the mechanism of particle collision and particle size. 
In the case of perikinetic aggregation of small particles, Smoluchowski calculated the number of collisions, $J_{i j}$, for the free diffusion of spheres of type $j$ and type $i$, occurring in unit volume per unit time:

$$
J_{i j}=4 \pi r_{i j} D_{i j} n_{i} n_{j}
$$

where $r_{i j}$ is the collision radius for particles of type $i$ and $j$, which is the center to center distance at contact. This can be assumed to be simply the sum of particle radii $a_{i}$ and $a_{j}$ :

$$
r_{i j}=a_{i}+a_{j}
$$

The term $D_{i j}$ is the appropriate mutual diffusion coefficient which takes into account the cumulative diffusion of the particles of type $j$ and $i$, since both are subject of Brownian motion:

$$
D_{i j}=D_{i}+D_{j}
$$

Based on these considerations Smoluchowski derived an analytical expression for the rate constant of diffusion limited aggregation of $i$ - and $j$-type particles:

$$
k_{i j}=\frac{2 k_{B} T}{3 \eta} \frac{\left(a_{i}+a_{j}\right)^{2}}{a_{i} a_{j}}
$$

where $\eta$ is the viscosity of the suspending liquid.

Assuming that collisions between particles/aggregates of approximately equal size dominate, then the collision rate constant becomes independent of particle size:

$$
k_{i j} \approx \frac{8 k_{B} T}{3 \eta} \equiv k
$$

Hence, in the very early stages of agglomeration of monodispersed particles the rate of decrease of total particle concentration $\left(n_{\text {tot }}=\sum_{p=1}^{\infty} n_{p}\right)$ can be calculated by summing the rates of change of concentrations of various aggregates:

$$
\frac{d n_{t o t}}{d t}=-\frac{k}{2} n_{t o t}^{2}
$$

where $k$ represents all of the rate constants, assumed to be equivalent. Integration of equation (22) gives the decrease of total number density $\left(n_{\text {tot }}=\sum_{p=1}^{\infty} n_{p}\right)$, as a function of time $t$ : 


$$
n_{\text {tot }}=\frac{n_{0}}{1+\frac{k n_{0}}{2} t}
$$

where $n_{0}$ is the initial concentration of primary particles, i.e. $n_{0}=n_{\text {tot }}$ at $t=0$. Equation (23) shows that after a characteristic time, $\tau=2 / \mathrm{kn}_{0}$, the total number of particles in solution is reduced to $1 / 2$ their initial number. Accordingly, equation 16 can be expressed as:

$$
n_{\text {tot }}=\frac{n_{0}}{1+t / \tau}
$$

It can be seen from equation (24) that for the very early stages of aggregation $(t<\tau)$ the total number of particles decreases faster in time than for $t>\tau$. This can be understood by considering the decrease in the aggregation rate as the number of particles decreases during the aggregation process. Note that $\tau$ is independent of the stage of coagulation but it depends on the initial particle concentration. Accordingly, for dilute suspensions $\tau$ will be very large and diffusion limited aggregation would require long times to achieve a large degree of aggregation.

It should be stressed that this treatment of the aggregation process is based on the assumption that the aggregates formed by the agglomeration of spherical particles are also spherical. In reality, aggregates have a fractal structure, which means that their effective volume can be larger than the total volume of the primary particles. Nevertheless, the Smoluchowski theory predicts quite accurately the rates of aggregation and aggregate size distribution for early stages of rapid coagulation of diluted dispersions [ 31] [32]

So far, it has been assumed that all collisions lead to attachment, thereby disregarding the effect of electrostatic or/and steric repulsion forces. The magnitude and range of these forces determines whether attachment occurs and hence the collision efficiency $(\alpha)$. Conventionally we speak of stability ratio $(W)$ instead of collision efficiency. These two quantities are directly related as follows:

$$
W=\frac{1}{\alpha}
$$

The stability ratio can be also expressed as the ratio of rapid coagulation to that found when a repulsive energy barrier hinder the close approach of colliding particles, i.e.

$$
W=\frac{k_{r}}{k_{s}}
$$

where $k_{r}$ corresponds to the rate constant of rapid- and $k_{s}$ of slow coagulation. 
Fuchs [33] developed a theoretical expression relating the stability ratio to the potential energy of electrostatic interactions by modifying the diffusion equation to account for the presence of interparticle interaction forces. Then, using equation (26) the stability ratio can be expressed as a function of interaction energy between identical particles with radius $a$ :

$$
W=2 a \int_{2 a}^{\infty} \exp \left(\frac{\Psi(r)}{k_{B} T}\right) \frac{d r}{r^{2}}
$$

The exponential term $\Psi / k_{B} T$ shows that if the interaction potential energy is sufficiently large in comparison to the thermal energy, measured by $\mathrm{k}_{\mathrm{B}} \mathrm{T}$, particles will be prevented from aggregation.

It is evident from equation (27) that in order to evaluate $W$ we need to integrate $\Psi$ over the entire separation range. These integral must be solved numerically because of the complicated form of the expression for the electrostatic and van der Waals interactions. However, the expression for $W$ can be considerably simplified by considering that the major contribution to the integral comes from $\Psi_{\max }$, i.e. the height of the potential energy barrier at the maximum. Based on these considerations, Verwey and Overbeek [19] obtained an approximate solution of equation (27) which allows understanding the behavior of $W$ regarding the barrier against coagulation and dispersion properties:

$$
W=\frac{1}{4 a}\left(\frac{2 \pi k_{B} T}{-\left(\frac{\partial^{2} \Psi}{\partial r^{2}}\right)_{\max }}\right)^{1 / 2} \exp \left(\frac{\Psi_{\max }(r)}{k_{B} T}\right)
$$

Reerink and Overbeek [34] developed a more convenient but more approximate expression for equation (27):

$$
W \approx \frac{1}{2 \kappa a} \exp \left(\frac{\Psi_{\text {max }}(r)}{k_{B} T}\right)
$$

This equation allows for a quantitative prediction of the effect of ionic strength on stability ratio, following DLVO theory with some simplifying assumptions.

\subsubsection{Orthokinetic aggregation}

Particle aggregation under orthokinetic conditions can be greatly enhanced due to increased rate of collisions or mechanical destabilization of dispersions stable at rest. Mechanical destabilization can be achieved when the hydrodynamic force pushing particles 
together can overcome the repulsive interparticle force responsible for kinetic stability. On the other hand, if this hydrodynamic force exceeds the attractive force of contact existing aggregates will breakup. The process of formation and breakup of aggregates under flow conditions is a very complex phenomenon that depends on the relative magnitude and ranges of the forces.

\subsubsection{Orthokinetic collision frequency}

In this section the collision frequency for simple shear and extensional flows will be expressed on the basis of the Smoluchowski model. The derived expressions will be compared to demonstrate the effect of flow kinematics.

Smoluchowski [30] considered two particles of size $i$ and $j$, suspended in a liquid and exposed to a laminar shear flow. The $j$ particle is assumed to be a reference sphere with radius $a_{j}$, that is located at the origin of the coordinate system, placed in a Lagrangian reference frame moving with the flow. Assuming only binary collisions between spheres of size $i$ and $j$, the collision rate can be obtained by calculating the flux of $i$ particles toward the reference $j$-particle.

Another assumption made by Smoluchowski is that the interaction potential is purely attractive and the Brownian motion and hydrodynamic interactions can be neglected. Therefore, one can consider particles moving along the undisturbed streamlines of the bulk motion until they collide with another particle. The positions of a particle, relative to the reference particle, which assures that the van der Waals forces can attract and hold particles together, can be represented as a sphere centered at the origin of the coordinate system. This sphere is referred to as collision sphere with radius $r_{i j}=a_{i}+a_{j}$.

The velocity of the bulk motion, $\vec{v}$ varies linearly in the space and is described by constant deformation rate tensor $\widetilde{\mathrm{E}}$ and vorticity tensor $\widetilde{\Omega}$ :

$$
\vec{v}=\widetilde{\mathrm{E}} \vec{r}+\widetilde{\Omega} \vec{r}
$$

where $\vec{r}$ is the position vector of the second particle with respect to the reference particle. With the assumption that particles move along the streamlines corresponding to $\widetilde{\mathrm{E}}$ and neglecting the local flow disturbance in the vicinity of the particles, the number of contacts of $i$-particle with the reference $j$-particle per unit time should equal the product of flow rate into the collision sphere, $Q^{+}$, and the number density of $i$ particles, $n_{i}$,:

$$
J_{i}^{0}=Q^{+} n_{i}
$$

where $J_{i}^{0}$ denote the number of collisions that the reference particle experience. To calculate $J_{i}^{0}$ one needs to express $Q^{+}$as product of the velocity of the flow, $\vec{v}$, and the area 
$A^{+}$through which this flow takes place, referred to as upstream interception area. The relative velocity of a simple shear flow can be presented in the form:

$$
\vec{v}=\dot{\gamma} y \vec{e}_{x}
$$

where $\dot{\gamma}=d v_{x} / d y$ is the shear rate in a simple shear flow, $y$ is a distance in y-direction and $\vec{e}_{x}$ is the unity vector.

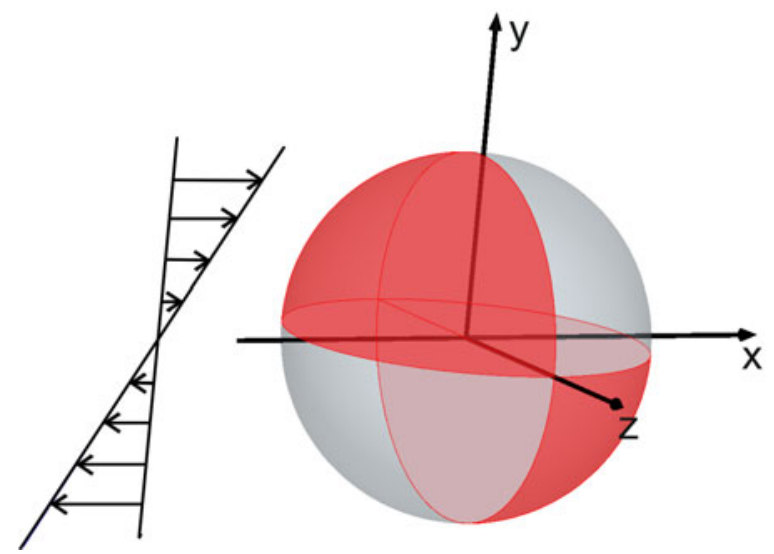

Figure 3. Schematic representation of the collision sphere with indicated in red upstream interception area $\mathrm{A}^{+}$in the case of simple shear flow

The upstream interception area $A^{+}$is the colored in red part of the collision sphere as represented schematically in Figure 3. Since the streamlines of the shear flow are straight lines in x-direction, the flow rate, $Q^{+}$, can be expressed as twice the flow rate through the semi-circle of the collision sphere $(x=0, y>0)$ :

$$
Q^{+}=2 \cdot \iint_{\substack{\text { semi } \\ \text { circle }}} \dot{y y d} A=2 \dot{y}_{s} \frac{\pi}{2} r_{i j}^{2}
$$

where $y_{s}=\frac{4}{3} \frac{r_{i j}}{\pi}$ is the center of mass of the semi-circle with radius $r_{i j}$. Hence the rate of flow into the area $A^{+}$at constant shear rate is given by:

$$
Q^{+}=\dot{\gamma} \frac{4}{3} r_{i j}^{3}
$$

Substituting equation (34) into (31), the number of contacts of $i$ particles with the reference particle in unit time becomes:

$$
J_{i}^{0}=\frac{4}{3} n_{i} r_{i j}^{3} \dot{\gamma}
$$

For $n_{j}$ reference $j$-particles, the well-known Smoluchowsi relation [30] results: 


$$
J_{i j}^{0}=\frac{4}{3} n_{i} n_{j} r_{i j}^{3} \dot{\gamma}
$$

where $J^{0}{ }_{i j}$ is the collision frequency between all $i$ - and $j$-particles per unit time and volume. Considering the second order kinetics of the aggregation process, the rate constant, $k_{i j}$, for the shear induced collisions becomes:

$$
k_{i j}=\frac{4}{3} \dot{\gamma}\left(a_{i}+a_{j}\right)^{3}
$$

This result demonstrates a strong dependence of shear induced collisions on the particle size. In contrast to the perikinetic collision rate constant, which is nearly independent of particle size for particles of roughly equal size, the orthokinetic collision rate is proportional to the cube of the collision radius $r_{i j}$ (sum of particle radii). This implies that larger particles have greater collision frequency and the aggregate growth rate increases as the aggregation proceeds.

In order to investigate the effect of flow kinematics on the rate of rapid flow induced aggregation, we can express the collision frequency for the case of arbitrary kinematics. Applying the same assumptions as in the Smoluchowski model, the collision frequency of spherical particles is analogues to equation (31). Hence, only an appropriate expression for $Q^{+}$, which accounts for the flow type, is needed to compute the rate of collisions. In general the flow rate, $Q^{+}$can be expressed as follows:

$$
Q^{+}=\iint_{A^{+}}(-\vec{v} \cdot \vec{n}) d A
$$

where $\vec{n}=\vec{r} / r_{i j}$ is the normal unit vector. For the upstream interception area, $A^{+}$, through which the flow takes place, the following criterion holds:

$$
-\vec{v} \cdot \vec{r} \geq 0
$$

Note that $-\vec{v} \cdot \vec{r}=0$ at the boundaries of $A^{+}$. Furthermore, considering equation (30), the criterion (39) can be written as:

$$
(-\widetilde{\mathrm{E}} \vec{r}) \cdot \vec{r} \geq 0
$$

In the principal coordinate system (with coordinate axes: $x_{1}, x_{2}, x_{3}$ ) the symmetric tensor $\widetilde{\mathrm{E}}$ for an incompressible flow, can be generally expressed in the form: 


$$
\widetilde{\mathrm{E}}=\left(\begin{array}{ccc}
p & 0 & 0 \\
0 & q-p & 0 \\
0 & 0 & -q
\end{array}\right) \quad \text { with } \quad \begin{aligned}
& p \geq 0 \text { and } q \geq 0 \\
&
\end{aligned}
$$

Note that the $x_{1}$ direction is towards the highest positive eigenvalue of $\widetilde{\mathrm{E}}$ and $x_{3}$ towards the smallest.

Considering the allowed parameter region, we can identify the following flow types: uniaxial extension when $p=2 q$, planar extension when $p=q$ and equal biaxial extension when $p=q / 2$. Furthermore, a general shear rate, $\dot{\gamma}$, can be assigned to the deformation rate tensor $\widetilde{\mathrm{E}}$, defined as follows:

$$
\dot{\gamma}=\sqrt{2 \cdot \operatorname{tr} \widetilde{\mathrm{E}}^{2}}=2 \sqrt{p^{2}+q^{2}-p q}
$$

where $t r$ stands for trace. Using equation (41) the equation (40) reduces to:

$$
-p x_{1}^{2}-(q-p) x_{2}^{2}+q x_{3}^{2} \geq 0
$$

Considering that:

$$
x_{1}^{2}+x_{2}^{2}+x_{3}^{2}=r_{i j}^{2}
$$

it is evident that the points $x_{1}=x_{2}=0$ and $x_{3}= \pm r_{i j}$ belong to the upstream interception area $A^{+}$. The " \pm " sign accounts for the north and south pole of the collision sphere.

Through elimination of the $x_{3}$-variable from equation (43) and (44), the projection of the $A^{+}$boundaries on the $x_{1}-x_{2}$ plane can be expressed as follows:

$$
\frac{p+q}{q r_{i j}^{2}} x_{1}^{2}+\frac{2 q-p}{q r_{i j}^{2}} x_{2}^{2}=1
$$

which is the equation of an ellipse with length of the semi major and semi minor axis:

$$
r_{i j} \cdot \sqrt{\frac{q}{p+q}} \quad \text { and } \quad r_{i j} \cdot \sqrt{\frac{q}{2 q-p}}
$$

These considerations show that in a principal coordinate system $A^{+}$consists of two parts, which are symmetric with respect to the $x_{1}-X_{2}$ plane and the mutual projection of the $A^{+}$boundaries is an ellipse (equation (45)). Furthermore, the points corresponding to $x_{3}= \pm r_{i j}$ belong to the both parts of $A^{+}$. 
Because of the equal velocity, $-\vec{v} \cdot\left(\vec{r} / r_{i j}\right)$, at points symmetric with respect to the $x_{1}-x_{2}$ plane, the flow rate $Q^{+}$can be computed as twice the flow rate through one of the two symmetrical parts of $A^{+}$. Taking that part of $A^{+}$which contains the point $x_{3}=+r_{i j}$ (indicated as $A_{x_{3}>0}^{+}$), the equation (38) can be written as follows:

$$
Q^{+}=2 \iint_{A_{x_{3}}^{+}>0}\left(p x_{1}^{2}+(q-p) x_{2}^{2}-q x_{3}^{2}\right) d A
$$

As mentioned above, in the special case of equal biaxial extension in the $x_{1}-X_{2}$ plane, the continuity equation is satisfied when $p=q / 2$. Consequently, equation (45) transforms to the equation of circle:

$$
x_{1}^{2}+x_{2}^{2}=\frac{2 r_{i j}^{2}}{3}
$$

with radius $s=r_{i j} \sqrt{2 / 3}$. Herewith, $A_{x_{3}>0}^{+}$represents a spherical cap around the point $x_{3}=$ $r_{i j}$ (Figure 4), the circular cross-section of which has a radius $s$ and intercept the $x_{3}$ axis at the point $x_{3}=r_{i j} / \sqrt{3}$. Hence the flow rate through the circular cross-sectional area of the spherical cup, $A_{x_{3}>0}^{+}$, can be represented as follows:

$$
\frac{Q^{+}}{2}=\frac{2 \pi r_{i j}^{2}}{3} \cdot q \cdot \frac{r_{i j}}{\sqrt{3}}
$$

where $q r_{i j} / \sqrt{3}$ represents the normal velocity component. Considering the expression of the generalized shear rate in the case of biaxial extension:

$$
\dot{\gamma}=2 \sqrt{p^{2}+q^{2}-p q}=q \sqrt{3}
$$

it follows

$$
Q^{+}=\frac{4 \pi}{9} r_{i j}^{3} \dot{\gamma}
$$




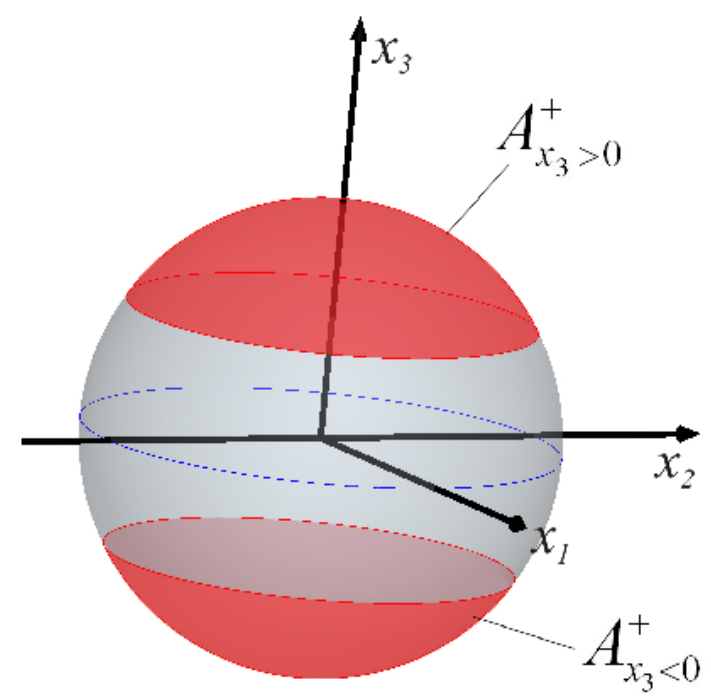

Figure 4. Schematic representation of the collision sphere with indicated in red upstream interception area $A^{+}$in the case of equal biaxial extension

For uniaxial extensional flow with principal elongation along the $x_{1}$ axis $(p=2 q)$ equation (45) reduces to a straight line with $x_{1}= \pm r_{i j} / \sqrt{3}$. The highlighted part of the collision sphere in Figure 5 represents the area $A^{+}$in this case. The boundaries of $A^{+}$are two symmetrical circles with centre at $x_{1}= \pm r_{i j} / \sqrt{3}$ respectively and radius $s=r_{i j} \sqrt{2 / 3}$. Since the flow exits $A^{+}$through these circular areas, the flow rate can be expressed as follows:

$$
Q^{+}=\frac{4 \pi}{3 \sqrt{3}} r_{i j}^{3} p
$$

Taking into account the generalized shear rate:

$$
\dot{\gamma}=p \sqrt{3}
$$

equation (51) result in equation (50).

In the case of planar extension $(p=q)$ the flow field corresponds to superposition of a simple shear flow and solid body rotation. Hence, the flow rate $V^{+}$for planar extensional flow can be expressed with equation (34). 


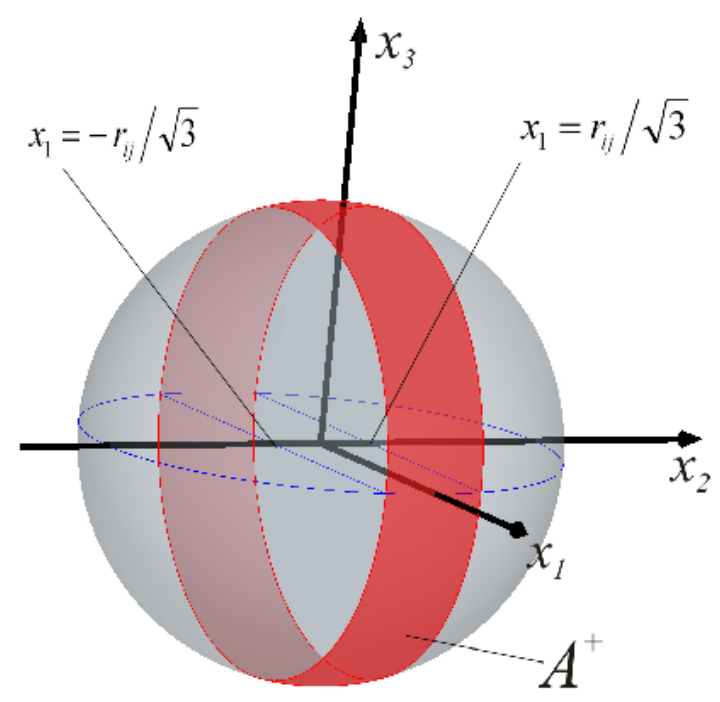

Figure 5. Schematic representation of the collision sphere with indicated in red upstream interception area $\mathrm{A}^{+}$in the case of uniaxial extensional flow

In summary, the number of particle collisions per unit time and volume for arbitrary flow kinematics is given by:

$$
J_{i j}^{0}=k_{f l} \frac{4}{3} n_{i} n_{j} r_{i j}^{3} \dot{\gamma}
$$

where $k_{f l}$ is a coefficient accounting for the flow kinematics. In the case of shear and planar extensional flow $k_{f l}=1$, while in the case of uniaxial and biaxial extension $k_{f l}=$ $\pi / 3$.

Using trajectory analysis, Zeichner and Schowalter [35] first compared the aggregation in simple shear flow with the aggregation in purely extensional flows. For an attractive potential and in the absence of hydrodynamic interactions, they found that the doublet formation in uniaxial extensional flow is higher by factor 3.62 than in simple shear flow that differs from the coefficient $k_{f l}$ predicted by the considerations presented above. This is due to the different equations used to express the generalized shear rate, which in both cases is proportional to the second invariant of the deformation rate tensor $\widetilde{E}$ but differ by a prefactor $2 \sqrt{3}$. However, it is obvious that the influence of the flow kinematics on the flow induced collision rate is small.

\subsubsection{Orthokinetic collision efficiency}

So far we have considered the Smoluchowski collision model in which both colloidal forces and hydrodynamic interactions are neglected. More recent studies have considered that the aggregation probability of colliding particles is determined by the forces acting on them. For negligible inertia $(\operatorname{Re}<<1)$, these forces are: a shear force exerted by the external flow on a colliding particle and transmitted to the second particle, a hy- 
drodynamic force due to the relative motion of particles and a colloid interaction force [36]. The effect of these interaction forces is considered in terms of orthokinetic collision efficiency $(\alpha)$, therefore the expression for the shear induced aggregation rate of unequal particles becomes:

$$
J_{i j}=J_{i j}^{0} \alpha_{i j}=\frac{4}{3} \alpha_{i j} n_{i} n_{j} \dot{\gamma}\left(a_{i}+a_{j}\right)^{3}
$$

Hydrodynamic forces have a significant influence on particle aggregation since lubrication stresses generated at small separations resist the approach of colliding particles such that they deviate from the linear trajectories assumed by Smoluchowski. A comprehensive treatment of the collision efficiency involves computing the relative trajectories of particles incorporating both hydrodynamic and colloidal interactions. Van de Ven and Mason [37] considered pure orthokinetic doublet formation of spheres in a laminar shear flow and computed the orthokinetic collision efficiency of equal-sized particles in the absence of electrical repulsive forces. The computed results are well represented by the following approximate semi-empirical formula:

$$
\alpha_{i i}=f(\lambda / a)\left(\frac{A_{H}}{36 \pi \eta \dot{\gamma} a^{3}}\right)^{0.18}
$$

in which the parameter $f$ depends on of the London wavelength $(\lambda)$ and particle size (a). Furthermore, Zeichner und Schowalter [35] emphasized that vorticity and deformation rate separately affect flocculation. They pointed out that vorticity in the undisturbed shear flow leads to closed trajectories in which particles orbit one another such that they leave the region where van der Waals forces are dominant and then return again. These recirculating streamlines limit the time available for the hydrodynamic and colloidal forces to interact, thus decreasing the aggregation efficiency. Hence the collision efficiency is higher in a purely extensional flow (infinite total strain) in comparison to pure shear flow (large total rotation). It has also been demonstrated that the difference between aggregation efficiency in extensional flow and the one in pure shear flow becomes higher as the shear rate increases [38]. Greene et al. [39] extended the results of Zeichner and Schowalter to different extensional flow fields, containing different amount of vorticity, and demonstrated that the aggregation efficiency is almost unaffected by the flow type, except for flow types similar to the simple shear. More recently Brunk et al. [40] [41] modeled the particle aggregation in turbulent conditions and systematically investigated the role of rotation on the coagulation rate. They confirmed the results of Zeichner and Schowalter and showed that stability ratio $(W)$ is proportional to $\dot{\gamma}^{0.23}$ in simple shear and $\dot{\gamma}^{0.11}$ in uniaxial extensional flow in the case of dilute suspensions with attractive interactions and considering the hydrodynamic interactions. 
Van de Ven \& Masson [42] as well as Zeichner and Schowalter [43] extended their results to the case of significant repulsive forces and demonstrated the interplay between colloidal and hydrodynamic interactions of flowing suspensions. They presented the results in stability diagrams characterizing the stability of dispersion as a function of relative strength of interparticle and hydrodynamic forces. The stability diagrams were divided into three regions: primary minimum coagulation, secondary minimum coagulation and a region where dispersion should be shear stable. When the van der Waals attraction dominates, the collision efficiency is found to be approximately proportional to $\dot{\gamma}^{0.77}$, but when repulsive interactions are dominant, the collision efficiency is zero and the dispersion is stable [35]. If both electrostatic repulsion and van der Waals attraction are important, no simple relationship exists. Suspensions may be stable over a certain range of shear rates, but aggregate at higher and lower values. Generally, if a suspension with a secondary minimum in the potential energy curve is sheared at a gradually increasing shear rate, the hydrodynamic force first pulls particles out from the secondary minimum, then pushes them over the repulsive barrier into the primary minimum and finally redisperses them. The hydrodynamic force must overcome the attractive force to pull a pair out of the primary minimum. Particles remain stable when the hydrodynamic force exceeds the maximum attraction in the secondary minimum but cannot overcome the repulsive barrier. The controlling dimensionless parameters in shear flow, used to estimate the relative influence of colloidal, hydrodynamic and diffusion forces are listed in Table 1.

Table 1. Nondimensional parameters [14]

\begin{tabular}{ll}
\hline Parameter & Description \\
\hline$P e=\frac{6 \pi \eta a^{3} \dot{\gamma}}{k T}$ & $\begin{array}{l}\text { ratio of the time scale of convective transport due to shear over the } \\
\text { time scale of diffusive transport }\end{array}$ \\
$N_{f}=\frac{6 \pi \eta a^{3} \dot{\gamma}}{A_{H}}$ & $\begin{array}{l}\text { measure of the strength of hydrodynamic forces relative to van } \\
\text { der Waals forces }\end{array}$ \\
$N_{r}=\frac{\varepsilon \psi_{0}^{2} a}{A_{H}}$ & $\begin{array}{l}\text { measure of the strength of electrostatic repulsive forces relative } \\
\text { to van der Waals forces }\end{array}$
\end{tabular}

Adler was the first to apply the theory of hydrodynamic interactions to the aggregation of unequal sized spheres exposed to shear flow [44] [45] [46]. He found that the homocoagulation of large particles in a pure shear flow is favored over their heterocoagulation with smaller particles, since the trajectory of smaller particles around a larger one passes at a minimum distance greater than the range of van der Waals attraction [47]. This is due to the hydrodynamic interactions, which cause a significant deviation of particle trajectories from the streamlines of the basic flow when two parti- 
cles of different size approach each other. The subject of heterocoagulation was analyzed further by Wang [48], who pointed out that heterocoagulation between particles of different size is favored over the homocoagulation for conditions of secondary minimum coagulation. In the case of particles of different material and different size, the size difference may also induce a secondary shear heterocoagulation between larger but less stable particles with smaller but more stable particles if the stability difference is not very large and the particle size difference is not too small. However, Brownian motion and the porous nature of aggregates may modify Adler's and Wang's conclusions.

Orthokinetic collision efficiency depends not only on hydrodynamic and colloidal interactions, but also the collision duration or contact time, i.e. the time spent by two particles in immediate vicinity [49]. Two simple models, which consider the effect of contact time on aggregation probability, will be discussed in this thesis. The first model predicts a critical contact time based on the particle residence time distribution within a collision sphere with radius comparable to the length scale of the van der Waals forces. The second model was proposed by Chesters [49]. and is based on the comparison of contact time with the time required for drainage of the fluid in the small gap between approaching particles to a critical separation at which the attractive interactions become comparable with the hydrodynamic force.

In order to elucidate the notion of contact time and to derive an expression for the critical contact time required for aggregation to occur, we will consider two-body collisions of equal particles with attractive interactions in simple shear flow, neglecting the effect of hydrodynamic interactions. Thus, it is convenient to consider again a collision sphere with radius $r=2 a$, which is around the reference particle and compute the mean residence time $(\bar{T})$ of particles in the collision sphere. Under laminar flow conditions for the case where the undisturbed velocity of the flow field is a linear function of position the flow field in the collision sphere is given by equation (30). Hence, $\bar{T}$ can be expressed as the ratio of the collision sphere volume $\left(V_{s}\right)$ to the total amount of fluid which enters in the collision sphere per unit time $\left(Q^{+}\right)$:

$$
\bar{T}=\frac{V_{s}}{Q^{+}}
$$

Substituting the correspondent expression for $Q^{+}$, derived in section 2.3.2.1 for different flow types, in equation (56) we find an inverse dependence of the mean residence time on the shear rate. For a simple shear and planar extensional flow $\bar{T}$ is:

$$
\bar{T}=\frac{1}{\dot{\gamma}}
$$


The mean residence time can be referred to as interparticle contact time. It represents the time available for the van der Waals forces to cause permanent binding of colliding particles and thus influences the collision efficiency $(\alpha=f(\bar{T}))$. Taking into account that $\alpha$ represents the fraction of successful collisions it will approach zero at very small $\bar{T}$ and reach unity at very long contact times for purely attractive systems. In the intermediate time domain there is a critical contact time required for the permanent attachment of colliding particles, which is material dependent and associated with a corresponding critical shear rate $\left(\dot{\gamma}_{c}\right)$. Considering the opposite effect of shear rate on collision efficiency compared to collision frequency, the aggregation rate is expected to pass through a maximum as the shear rate further increases. At small shear rates, the collision efficiency is close to unity and almost every collision leads to aggregation, but the collision frequency is low and very few aggregates will be formed. Despite the large collision frequency at very large values of $\dot{\gamma}$, the aggregation rate is low due to the small collision efficiency.

In order to express the aggregation rate as a function of a critical contact time, we consider the contact time distribution in the collision sphere with radius $r$. For the case of a simple two-dimensional shear flow with shear rate:

$$
\dot{\gamma}=d v_{x} / d y
$$

the distribution of the contact time $(T)$ in the collision sphere (Figure 6) is given by:

$$
T_{\text {contact }}(y)=\frac{2 x(y)}{v_{x}(y)}=\frac{2 \sqrt{r^{2}-y^{2}}}{\dot{\gamma y}}
$$

where $x(y)$ is the path travelled by a particle in the collision circle with a relative velocity $v_{x}(y)$ depending on the position of particles on the $y$-axis and $y$ represents the distance on the $y$-axis. The factor of 2 is introduced to account for the symmetry of the flow. 


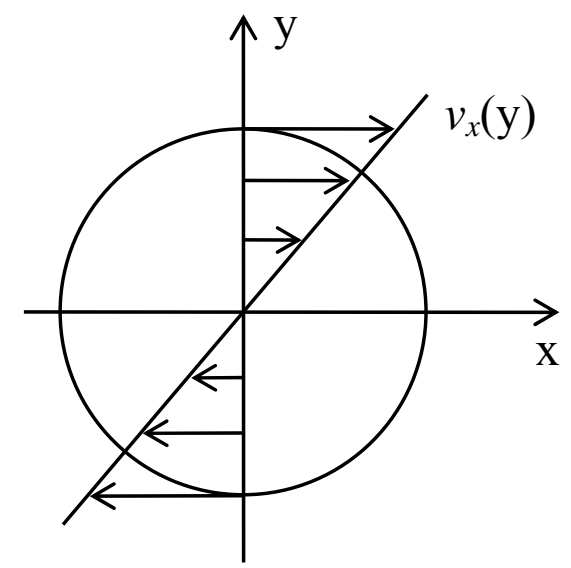

Figure 6. Schematic illustration of the collision sphere with radius $r$ at the origin of the coordinate system exposed to simple shear flow with $\dot{\gamma}=d v_{x} / d y$.

Equation (59) shows that the contact time $\left(T_{\text {contact }}\right)$ decreases with increasing the interparticle separation distance $y$ and reaches zero at $y=r$. Hence, depending on the range of attractive interactions $r$, a critical contact time $T_{c}$ exists which is associated with a critical separation distance $y_{c}$. The critical contact time can now be expressed in analogy to equation (59) in the form:

$$
T_{c}^{2}=\frac{4\left(r^{2}-y_{c}^{2}\right)}{\dot{\gamma}^{2} y_{c}^{2}}
$$

Assuming that for particles flowing into the collision sphere with radius $y_{c}$, i.e. in the case of residence time larger than $T_{c}$ the aggregation probability is unity, the doublet formation $(J)$ can be expressed as follows:

$$
J=Q_{y<y_{c}} n^{2}
$$

where $Q_{y<y_{c}}$ is the flow rate into the sphere with radius $y_{c}$ :

$$
Q_{y<y_{c}}=2 \int_{0}^{y_{c}} v_{x}(y) b d y
$$

where $b$ is the length perpendicular to the two-dimensional shear plane and is proportional to the radius $r$. Introducing equation (58) and (60) in equation (62) we get:

$$
Q_{y<y_{c}}=b \frac{4 r^{2} \dot{\gamma}}{T_{c}^{2} \dot{\gamma}^{2}+4}
$$

Then, using equation (61) and equation (63), the total number of doublets $(N)$ formed per unit time in a laminar shear field, at constant shear rate, can be expressed as: 


$$
N=\int_{0}^{t} J d t=\frac{4 b n^{2} r^{2}}{T_{c}^{2} \dot{\gamma}^{2}+4} \dot{\gamma} t
$$

For the application of this formula appropriate expression for $T_{C}$ is necessary. Furthermore, it is based on a simple theoretical model, which neglects the hydrodynamic interactions. This model concerns only the contact time in comparison to the time required for particles to approach each other due to van der Waals forces and form a permanent doublet. In reality, hydrodynamic interactions displace spheres from the linear trajectories, and accordingly, affect the separation distance of approaching particles. Nevertheless, equation (64) serves to demonstrate that the aggregation rate is inversely proportional to the critical contact time required for aggregation to occur.

Chesters [49] considered the lubrication forces during particle collision and pointed out that aggregation will occur if the time spent by two particles in immediate vicinity $\left(t_{\text {contact }}\right)$ exceeds the time required for drainage $\left(t_{\text {drainage }}\right)$ of the fluid in the small gap between approaching particles to a critical separation $\left(\mathrm{h}_{\mathrm{c}} \sim\left(\mathrm{A}_{\mathrm{H}} / 72 \pi \eta \mathrm{a} \dot{\gamma}\right)^{1 / 2}\right)$ at which the van der Waals attraction becomes comparable with the hydrodynamic force. Hence, the aggregation probability $\mathrm{P}$, providing the first indication of whether approaching particles will stick together or not, can be expressed as an exponential probability function of the ratio between $t_{\text {drainage }}$ and $t_{\text {contact }}$ :

$$
P \approx \exp \left(-\frac{t_{\text {drainage }}}{t_{\text {contact }}}\right)
$$

The drainage time $t_{\text {drainage }}$ has been expressed as the time required to squeeze out the fluid between particles from the separation at which the drainage law becomes relevant $\left(\mathrm{h}_{\mathrm{o}} \sim \mathrm{a} / 4\right)$ to the critical separation, $\mathrm{h}_{\mathrm{c}}$. Together with the correlation $\mathrm{t}_{\text {contact }} \sim \dot{\gamma}^{-1}$, the following expression for the estimation of the aggregation probability has been formulated:

$$
P \sim\left(3 N_{f} / 4\right)^{-1 / 8}
$$

where $N_{f}$ is the flow number as listed in Table 1.

The mathematical considerations for the contact time distribution in the collision sphere assume existence of a critical contact time, while the Chester's model relates this time to the drainage time considering the lubrication force resisting the close approach of two spherical particles in near contact.

The theoretical analysis reported above focuses on the aggregation dominated by the convection but in many cases Brownian motion of particles has a significant influence on shear-induced aggregation. The effect of Brownian motion on the aggregation in shear as well as in elongational flow was considered by Zechner and Schovalter [35], 
van de Ven and Masson [50], Feke and Schowalter [51] [52] and more recently by Melis et al. [53]. Van de Ven and Masson [50] developed perturbation analysis of the relevant pair probability equation with respect to the Peclet number $(\mathrm{Pe})$ as a parameter accounting for the ratio between convective and Brownian motion. Their analysis was limited to very small Pe. It was found that convection increases the aggregation rate since the flow brings the particles together and increases the number of collisions. The results of their analysis can be presented in the form:

$$
J=\frac{8 n^{2} k_{B} T}{3 \eta W}\left(1+0.257 \frac{P e^{1 / 2}}{W}\right)
$$

Feke and Schowalter [51] [52] considered the case when the shear induced coagulation is the primary but not the only contribution to aggregation, including weak Brownian motion (high but finite $P e$ ). They developed a perturbation analysis of the pair probability equation for the relative motion of two particles with regard to diffusion and convection terms and came to the results that a little Brownian motion can either enhance or reduce the rate of rapid shear induced aggregation, depending on the flow number $N_{f}$. Furthermore Brunk et al [40] pointed out that decreasing the shear rate while keeping $N_{f}$ constant, the aggregation efficiency for dilute suspensions with attractive interactions increases, since approaching particles will have many close encounters due to the Brownian motion, before they either collide or the flow carries them away from each other. On the other hand, at large total strain colliding particles have basically one opportunity for successful collision before the flow separates them.

\subsection{Criteria for stability of flowing colloid suspensions}

We have covered the most important aspects of flow induced aggregation, but it is convenient to summarize in this separate section the criteria for mechanical stability of flowing suspensions. In the majority of the work on this problem [14], the attention has focused on limits of stability neglecting the Brownian motion, considering only the force balance by collision. When two particles are brought together as a result of velocity gradient the probability of doublet formation is determined by the forces acting on them. Thus, the question of flow stability depends on the magnitude of hydrodynamic force $F_{H}$ pushing particles together or apart depending on the magnitude of DLVO forces. As discussed in section 2.2.3 the total energy of interaction between particles has typically three extrema in the force:

$$
\text { primary minimum: } \quad F_{p}=-\left.\frac{d \Psi}{d h}\right|_{\min }<0
$$


secondary minimum: $F_{s}=-\left.\frac{d \Psi}{d h}\right|_{\mathrm{sec}}<0$

repulsive barrier: $\quad F_{r}=-\left.\frac{d \Psi}{d h}\right|_{\max }>0$

Aggregation in the primary minimum (permanent doublet formation) will occur when the hydrodynamic force, $F_{H}$, can overcome first the attraction in the secondary minimum, $-\mathrm{F}_{\mathrm{s}}$, and then the maximum repulsive force $F_{r}$. But if $F_{H}$ exceeds the attractive force in the primary minimum $\left(-F_{p}\right)$, the doublets will deflocculate. For secondary minimum aggregation the attractive force $\left(-F_{s}\right)$ must be greater than the hydrodynamic force $F_{H}$. Hence, the criteria for flow stability are:

$-F_{s}<F_{H}<F_{r} . \quad$ and $\quad F_{H}>-F_{p},-F_{s}, F_{r}$.

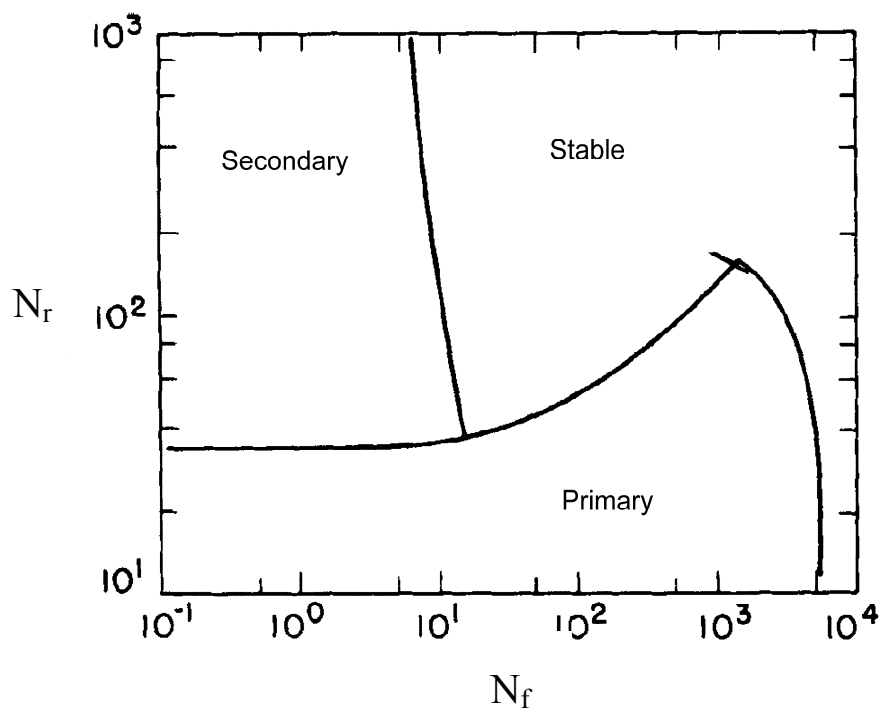

Figure 7. Stability plane for two spherical particles in a simple shear flow, with unretarded attraction and constant surface potential boundary conditions [35]

According to these considerations we can use the nondimesional groups $\mathrm{N}_{\mathrm{f}}$ and $\mathrm{N}_{\mathrm{r}}$ (Table 1) to characterize the relative strength of colloidal and hydrodynamic interactions and thereby define the stability state of a suspension. Results from the trajectory analysis for dilute suspensions with repulsive interactions [35][42] have been presented as stability diagram in the $\mathrm{N}_{\mathrm{f}}-\mathrm{N}_{\mathrm{r}}$ plane. Figure 7 shows a typical stability diagram. In the case of small $\mathrm{N}_{\mathrm{r}}$ values, there is no repulsive barrier and permanent aggregation in the primary minimum occurs in the whole $\mathrm{N}_{\mathrm{f}}$ range for which $\mathrm{F}_{\mathrm{H}}$ cannot surpass the attractive force at contact $\left(-F_{p}\right)$. For $\mathrm{N}_{\mathrm{f}}>10^{4}$, in this particular case, particle aggregation is not expected. For $\mathrm{N}_{\mathrm{r}}=100$, a repulsive barrier exist but at low values of $\mathrm{N}_{\mathrm{f}}$ the attractive force keep the particles in the secondary minimum. Increasing $\mathrm{N}_{f}$, particles first deflocculate out of the secondary minimum and then coagulate in the primary minimum. 
Hence, particles remain stable only in a certain intermediate range of shear rates, i.e. $\mathrm{N}_{\mathrm{f}}$. The width of this range depends on the $\mathrm{N}_{\mathrm{r}}$ value. At sufficiently large values of $\mathrm{N}_{\mathrm{r}}$, the hydrodynamic force cannot push particles over the repulsive barrier and the dispersion is stable with respect to primary minimum coagulation.

\subsection{Particle capture mechanisms}

The retention of small particles by stationary collector units during flow of liquid suspensions is of great interest for areas such as filtration [54] [55] [56], ground water contamination [57] [58], oil recovery [59] and technical operations like coating and pumping. This phenomenon is also relevant for the application of suspensions in emerging processing technologies based on microfluidic devices [60] [61] [62]. The present section concentrates on the particle retention during the flow of suspension through narrow channels. The discussion will be focused mainly on the possible capture mechanisms which can cause blockage of the channel and thus compromise the efficiency and reliable operation of processing technologies based on microfluidic devices and other technical processes.

During flow through porous media or narrow channels, suspended particles can interact with the channel walls in different ways that may lead to their retention. Potential mechanisms for clogging of microchannels by flowing colloidal suspension are fouling, i.e. particle deposition as a result of attraction to the wall surface, and plugging by aggregation in the flow field or by hydrodynamic bridging. We should also mention that another possible mechanism of channel clogging is straining or size exclusion at a channel constriction that is smaller in size than a particle. Flow induced aggregation has been discussed in detail in the preceding sections therefore we will concentrate here on the process of particle deposition and hydrodynamic bridging. Similar to flow induced aggregation, the deposition process is assumed to takes place in two separate steps: namely, a transport and an attachment/adhesion step. In the transport step, particles in the bulk suspension can be transported to the interface region as a result of inertial forces, interception, Brownian diffusion or sedimentation. The capture mechanism is termed inertial impaction when inertial forces cause particles to deviate from the streamlines and thus transport them toward the collector surface. A measure of the importance of particle inertia is the Stokes number $(S t)$. It is the ratio of viscous to inertial forces and is expressed by relating the convective time scale to a particle response time scale [14]: 


$$
S t=\frac{2}{9}\left(\frac{d}{d_{c h}}\right)^{2} \frac{\rho}{\rho_{f l}} \mathrm{Re}
$$

where $d$ is the particle diameter, $d_{c h}$ is the channel diameter, $\rho$ and $\rho_{f l}$ are particle and fluid densities, respectively, and $R e$ is the channel Reynolds number defined as follows:

$$
\operatorname{Re}=\frac{\rho_{f l} \bar{v} d_{c h}}{\eta}
$$

where $\bar{v}$ is the average velocity within the channel. When $S t<1$ the particle inertia is insignificant and inertial impaction is not possible even in the absence of electrostatic repulsion [63]. In fact, inertial impaction is an important mechanism in the case of aerosols but not for liquid suspensions.

Capture also occurs in the absence of particle inertia. Particles travelling on streamlines that pass within a characteristic distance, comparable to the length scale of the attractive interactions, from the channel wall can stick irreversible to the wall. The characteristic distance is closely related to the colloidal and hydrodynamic forces that control particle stability. This capture mechanism is known as interception and has been investigated recently by Wyss et al. [64] at the level of a single pore using microfluidic channels. They showed, experimentally, that a sticking event is determined by the initial streamline on which a particle is travelling and does not depend on flow rate and particle volume fraction.

For colloidal particles, Brownian motion can play a significant role in the transport of particles to the wall, similarly to the case of flow induced particle aggregation discussed in section 2.3.2.2. In the case of large or dense particles, sedimentation can be also responsible for particle deposition especially when the forced convection is weak i.e. at small Peclet numbers. Similar to flow induced aggregation, the efficiency of particle deposition by all these mechanisms is affected by the competition between attractive, electrostatic and hydrodynamic forces.

Hydrodynamic bridging refers to the phenomenon when particles smaller than the channel size arrive simultaneously and block the channel. Hydrodynamic bridging is effected by the colloidal repulsion force and the drag force during the low Reynolds number flow of electrostatically stabilized dispersions through pore constriction. The experimental results of Ramachandran and Fogler [65] demonstrate that the plugging phe- 
nomenon occurs when the flow velocity is higher than a critical velocity such that the hydrodynamic force overcomes the interparticle and particle-pore electrostatic repulsion. Note that extent of clogging due to hydrodynamic bridging increases with increasing the flow rate. Formation of particle bridge across the channel entrance by this mechanism is also strongly dependent on the particle concentration and the aspect ratio, defined as the ratio of channel size to particle size. 


\section{Experimental section}

This chapter describes the experimental apparatus and procedures applied for the investigation of flow induced aggregation and sample characterization. The materials comprise concentrated polymer suspensions, monodispersed particles and surfactants. The investigated highly concentrated suspensions will be characterized concerning their particle size distribution, stabilization mechanism and electrical properties

\subsection{Experimental apparatus and procedure}

In order to investigate experimentally flow induced aggregation in converging flow field we used a ring-slit device, which has already been established to characterize flowinduced aggregation for industrial quality control and product development purposes [1]. Single-particle optical sensing technique (SPOS) was applied for qualitative and quantitative analysis of micron-sized objects (e.g. aggregates of primary particles, impurities) extant in concentrated nano-particle suspensions, which have strong influence on the flow stability and slit clogging phenomenon. Shear flow aggregation experiments were performed using a cylindrical shear cell mounted to a rotational rheometer.

\subsubsection{Ring-slit device}

A schematic diagram of the ring-slit device is shown in Figure 8a. It consists of a capillary viscometer (Rosand RH2000) and a home-made stainless steel ring-slit die mounted to the bottom end of the wall of a cylindrical sample reservoir. The ring-slit geometry allows us to maintain a small gap height $(\mathrm{H}=10-30 \mu \mathrm{m})$, corresponding to a high contraction ratio $(1: 1000)$, and a large cross-sectional area at the same time. The large cross-sectional area prevents from immediate complete blockage of the slit by the formed aggregates, which can cause an enormous increase of the pressure and thus makes the sealing of the device very challenging. Indeed, measurements with a rectangular slit die with a slit cross-sectional area about 10 times smaller than ring-slit were extremely problematic regarding device sealing, particularly in the case of unstable dispersions. Furthermore it should be mentioned that the piston sealing has been achieved by using a polyetheretherketone (PEEK) piston tip (Malvern Instruments, Germany) instead of rubber o-rings. The technical specifications of the ring-slit device are summarized in Table 2 .

Flow-induced aggregation and corresponding aggregate retention at the slit entrance are examined by measuring the pressure drop across the ring-slit. The suspension is forced to flow through the ring-shaped slit at a constant volumetric flow rate $(Q)$, which is con- 
trolled by setting the piston speed $(v)$. The range of flow rates applied in this study was varied from $23 \mathrm{~mm}^{3} / \mathrm{s}$ to $1600 \mathrm{~mm}^{3} / \mathrm{s}$. A pressure transducer (Gefran Deutschland $\mathrm{GmbH})$ mounted above the die records the corresponding extrusion pressure $(p)$ as a function of time. If the suspension is stable the pressure adjusts itself to a comparably low constant value determined by the viscosity of the particular sample (Figure 8c). In this case the instrument operates as a high-shear capillary rheometer and apparent shear rates, $\dot{\gamma}_{a p p}$, apparent shear stresses, $\tau_{a p p}$, and viscosity, $\eta_{a p p}$, can be calculated according to $[66]$ :

$$
\dot{\gamma}_{a p p}=\frac{6 Q}{B \cdot H^{2}} \quad \text { with } \quad Q=\pi \cdot R^{2} \cdot v \quad \tau_{a p p}=\frac{p \cdot H}{2 L} \quad \eta_{a p p}=\frac{\tau_{a p p}}{\dot{\gamma}_{a p p}}
$$

(a) ring-slit device

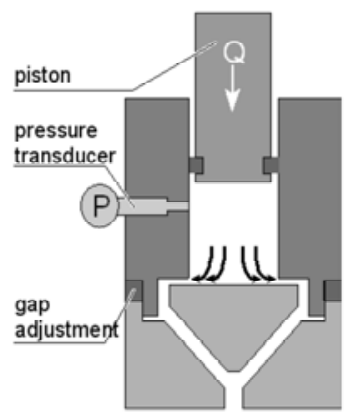

(b) slit entrance geometry

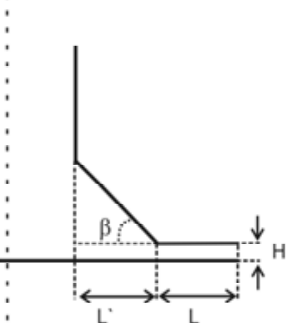

(c) extruded volume vs. pressure

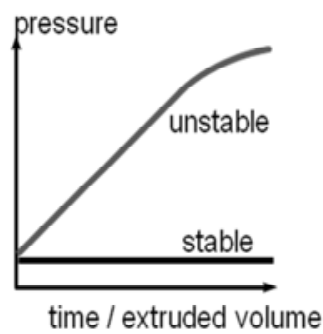

Figure 8. Ring-slit device (a), details of the slit entrance geometry (b) and pressure development for stable and unstable samples (c)

If the suspension is not stable, aggregates gradually clog the slit and the extrusion pressure increases with time (Figure 8c). The recorded pressure signal is normalized by the so-called viscous pressure $\left(\mathrm{P}_{\text {viscous }}\right)$, the initial pressure reading that corresponds to the viscosity of the particular sample, so that we only compare the pressure increase caused by the aggregate retention. All experiments are conducted at room temperature and samples are pre-filtered prior to the ring-slit tests in order to remove impurities and aggregates formed in previous processing steps. 
Table 2. Technical specifications of the ring-slit device

\begin{tabular}{ll}
\hline Specification & Value \\
\hline Radius $\mathbf{R}$ of cylindrical sample chamber & $12 \mathrm{~mm}$ \\
Gap height $\mathbf{H}$ & $11 \mu \mathrm{m}, 21 \mu \mathrm{m}$ and $26 \mu \mathrm{m}$ \\
Entrance gap width $\mathbf{B}=\mathbf{2} \pi \mathbf{R}$ & $62.8 \mathrm{~mm}$ \\
Gap length $\mathbf{L}$ & $2 \mathrm{~mm}, 1 \mathrm{~mm}$ and $0.8 \mathrm{~mm}$ \\
Entrance length $\mathbf{L}$ & $1 \mathrm{~mm}$ and $1.2 \mathrm{~mm}$ \\
Entrance angle $\boldsymbol{\beta}$ & $30^{\circ}, 45^{\circ}, 90^{\circ}$ \\
\hline
\end{tabular}

\subsubsection{Ring-slit die}

The ring-slit die is made of stainless steel (X5CrNi18-10) and comprises three component parts (Figure 9) mounted together with six M5 bolts. Torque wrench (Torcofix-SE 4100-00, Rahsol) was used to tighten the bolts evenly with $2 \mathrm{Nm}$ torque. The ring-slit is typically $1 \mathrm{~mm}$ long and arises from the gap between the circular surfaces of the top and bottom part highlighted in red in Figure 9. The height of the slit is adjusted by variation of the thickness of the ring mounted between the top and bottom part. The slit height is then verified by calibration measurements with standard silicon oil (Wacker Chemie AG, Germany) of known viscosity $(\eta=0.01 \mathrm{~Pa} \cdot s)$. The used silicon oil behaves as a Newtonian fluid at the shear rates applied (1300-5000 s${ }^{-1}$ ). Thus, knowing slit length and width, the piston speed and viscosity of the used silicon oil, the slit height is easily obtained from equations (73).

It is important to note that the ring-slit die allows for variation of the slit-entrance length $\left(L^{\prime}\right)$ and entrance angle $(\beta)$ as well as the length $(L)$ of the slit itself. The geometry of the entrance region is illustrated in Figure $8 \mathrm{~b}$ for the case of $45^{\circ}$ angle. Variation of the entrance angle has been performed by countersinking of the top part of the die with fluted countersink cutter (HSS, Format professional quality) having the desired angle.
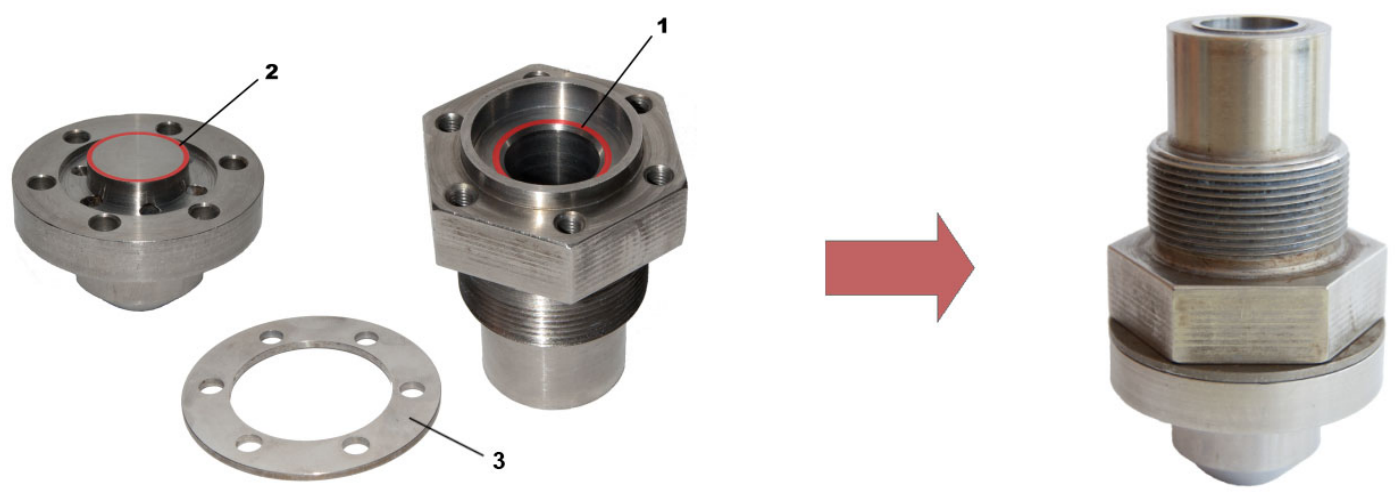

Figure 9. Ring-slit die, consisting of top (1) and bottom (2) part and a ring (3). The slit walls are highlighted in red. 
Coordinate measuring machine, Zeiss Prismo Navigator with VAST® universal probe, was used to characterize the planarity of the die surfaces which are involved in the slit formation. The profiles along the circumference of the slit walls are displayed in Figure 10 and reveal wall planarity within about one micron. The peaks in the bottom surface profile are attributed to artifacts caused by dust particles or fouling on the surface. Note that the surface planarity is achieved by surface grinding (Willy Lillich $\mathrm{GmbH}$, Germany) using Kellenberger Universal Grinder U600.
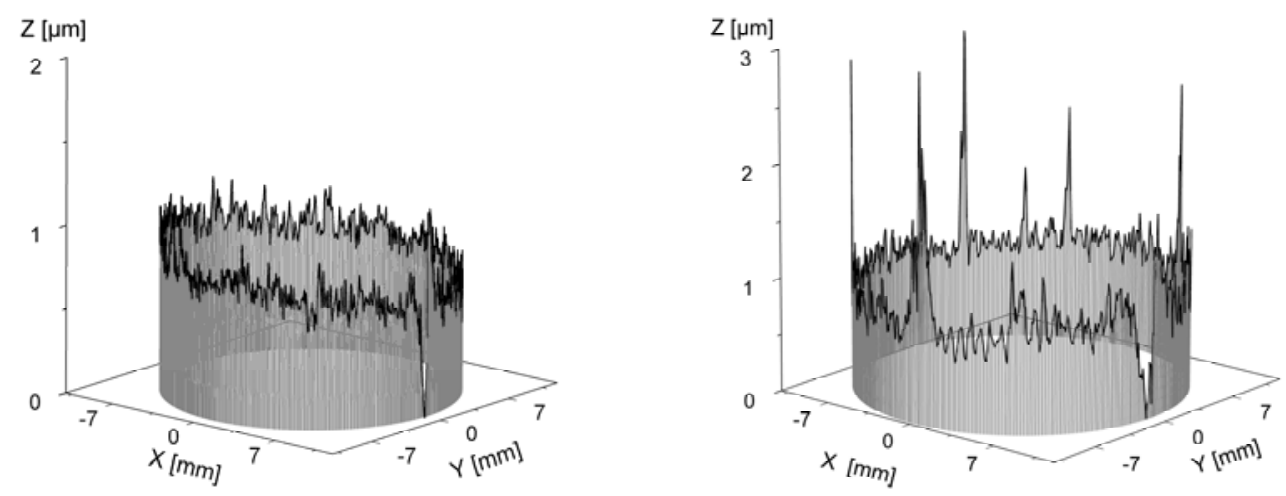

Figure 10. Wall planarity of the slit top- (a) and bottom-wall (b), highlighted in red in Figure 9 and measured by Zeiss Prismo Navigator with VAST ${ }^{\circledR}$ universal probe

Planarity of the die walls between which the ring is to be placed turned to be within about $2 \mu \mathrm{m}$ (Figure 11). The grinding of these walls has been performed by different procedure, which requires remounting of the die and can not assure perfect coplanarity. Planarity of the rings used for the adjustment of the slit height was examined by measuring its thickness with a digital micrometer gauge (TESA, 06030041) with $1 \mu \mathrm{m}$ resolution. The results revealed $\pm 1 \mu \mathrm{m}$ deviation in the thickness.

The planarity of the slit itself can not be measured in situ by direct observations; therefore the effect of small non-planarity of the walls between which the ring is placed has been examined by performing measurements varying the radial position of the die parts with respect to each other. For this purpose we extruded a highly concentrated polyurethane suspension through a $21 \mu \mathrm{m}$ slit. It turned out that the radial position of the two die parts with respect to each other has no influence on the flow stability of the investigated sample. These results suggest that the non-perfect coplanarity of the die walls that are actually not directly involved in slit formation, does not influence significantly the coplanarity of the slit walls. 

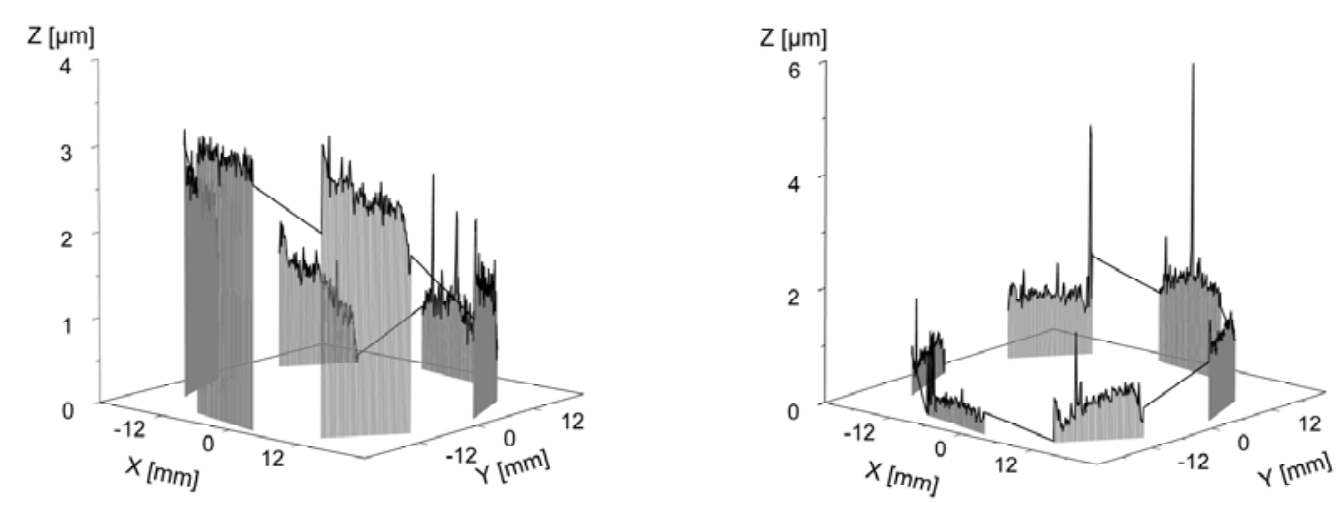

Figure 11. Planarity of the outer parts of the top- (a) and bottom-wall (b) between which the ring is placed, measured by Zeiss Prismo Navigator with VAST ${ }^{\circledR}$ universal probe

Q-Scope ${ }^{\mathrm{TM}}$ Scanning Probe Microscope (SPM) (Ambios Technology Inc.,USA) was used for the characterization of the slit surface roughness. The measurements were performed in Contact Mode using contact tips PPP-CONTR (Schaefer Technologie $\mathrm{GmbH}$ ). Typical SPM image of the slit surface is shown in Figure 12. The measured average surface roughness $\left(\mathrm{R}_{\mathrm{a}}=128.8 \mathrm{~nm}\right)$ reveals relative smooth surface. Note that smoother surfaces could be obtained only by manual polishing which lead to nonplanarity of the slit walls.

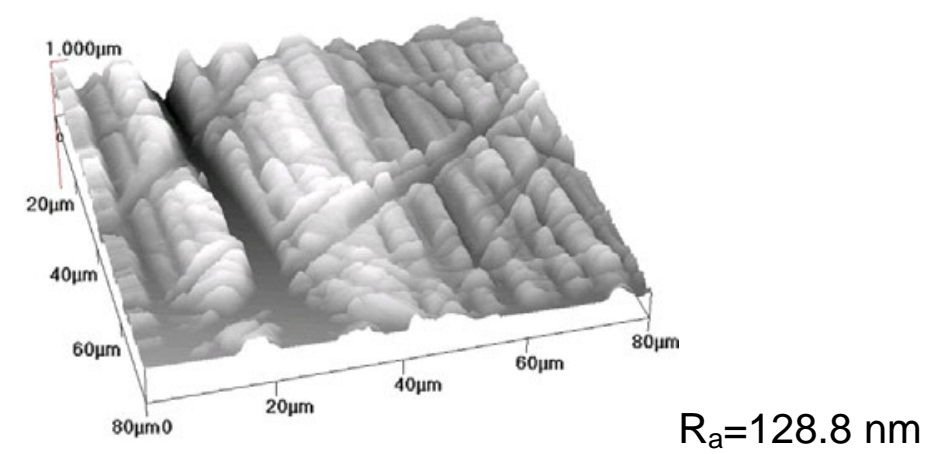

Figure 12. SPM images of a slit surface

One of the major challenges of this work was the manufacturing of the ring-slit die because of the microfluidic nature of the device. Although the precision of the metal polishing using classical mechanical processing techniques is in general about $1 \mu \mathrm{m}$, the surface coplanarity is limited by the used technique and procedure.

\subsubsection{Single-particle optical sizing (SPOS)}

There are a variety of particle size distribution analysis techniques, each with its own limitation. In this study we use a single-particle optical sizing technique (SPOS) to determine the size of individual micron-sized particles or aggregates extent in nanoparticle suspensions. The key difference between the SPOS method and the conventional instruments based on ensemble techniques is the high resolution and accuracy 
regarding small amounts of large particles besides the main population of primary particles. The SPOS technique has been already proved to give detailed information about aggregate size distribution and its change in time when particle aggregation occurs [67] [68] [69]. Furthermore, the SPOS method is often used to analyze small amount of large particles in silica slurries applied to chemical-mechanical polishing in the semiconductor industry [70] [71] [72] [73]. The SPOS technique allows counting of individual particles resulting in high sensitivity on the order of parts per billion.

\subsubsection{Description of the SPOS technique and procedure}

The SPOS measurements were carried out with the AccuSizer 780A (PSS Inc., Santa Barbara, CA) equipped with a model LE400-05ES particle sensor. The optical sensor is factory calibrated using size-certified polystyrene standards. Particle size distributions can be measured in the diameter range of $0.5-400 \mu \mathrm{m}$.

The instrument consists of: Autodiluter system, optical sensor, pulse-height analyser (PHA), computer processor and software controller. The Autodiluter system allows continuous dilution of the sample during the measurement. The PHA unit monitors the frequency of particles passing through the optical sensor and when particle concentration falls below a threshold known as the coincidence limit, the PHA unit begins data collection. Note that the autodilution mode has not been used in this investigation. We used gravity drain operation for quantitative analysis of the amount of micron-sized objects in the investigated nanosuspensions. For this purpose the volume of injected sample and vessel fill were adjusted prior to measurements so that the counts per millimeter (coincidence ratio) vary in the desired range.

The counting and sizing of particles by the AccuSizer is based on both light obscuration and light scattering. A laser beam is emitted across the flow channel in the sensor into a detector. As particles pass the sensor a fraction of the laser beam is blocked and a detector records the decrease in light intensity as change in the output voltage. The magnitude of this change in voltage is converted to particle size by comparing it with a calibration curve created by standard particles of known size. This analysis based on light obscuration is known as "extinction mode" and it is suitable for particles larger than 1 $1.5 \mu \mathrm{m}$.

The scattering-type optical sensor provides an additional resolution at the lower end of the particle size range. A single particle scatters light with an angular dependence, which depends on its diameter and refractive index. Thus differences in the refractive index must be corrected when the refractive index of measured particles differs from that of particles used for the calibration. However, this mode of analysis known as "summation mode" has not been used in this investigation, since we are primarily interested in particles larger than 1 micron. 
Samples, typically $10-100 \mu 1$, are withdrawn by pipette into the vessel filled with $80 \mathrm{ml}$ distilled, $0.22 \mu \mathrm{m}$ pre-filtered water. The water pre-filtration serves to reduce the amount of background contaminants. Samples were homogenized in the instrument vessel by moderate stirring for 1 minute prior to a measurement. Stirring was considerably decreased or stopped during data collection to avoid the formation of bubbles. Care was taken to avoid contamination and air bubbles during the measurements which can influence the quantitative analysis of investigated samples. The extent of sensor cleanliness was controlled by monitoring the baseline sensor voltage to ensure accurate operation. The baseline extinction voltage should not be below $12 \mathrm{~V}$ and the summation voltage should not be smaller that $10 \mathrm{~V}$, otherwise the sensor should be cleaned thoroughly.

Data collection was provided by the CW788-Nicomp software (PSS, Inc., Santa Barbara, CA), which accumulate the data in 512 logarithmically spaced channels from minimum to maximum size. The evaluation of raw particle counts per size channel allows for calculation of measured particle volume. Accordingly, by measuring a defined amount of the suspension the volume fraction of micron-sized particles can be obtained.

\subsubsection{SPOS accuracy and resolution}

In this section the accuracy of the SPOS method and the influence of potential error sources will be discussed. It will be shown that the sensor calibration, particle count rates, background counts (air bubbles and contaminations) can impact the sizing and counting accuracy. The influence of particle count rate on the volume recovery ratio and particle size distribution of polystyrene standard will be examined and optimum sizing conditions will be established. The instrument's sensitivity to small amount of micronsized particles in nanodispersions will also be investigated and it will be shown that the large amount of particles below the measuring range of the instrument does not influence the analysis of micron-sized impurities in nanodispersions.

\subsection{Maximum coincidence and sizing accuracy}

The SPOS method requires a strong dilution to avoid particle coincidences. The maximum particle count rate (expressed in particle per millilitre) should not exceed the coincidence limit of the sensor, in order to prevent multiple particles to pass the sensor simultaneously and thus to be recorded as a single large particle. 


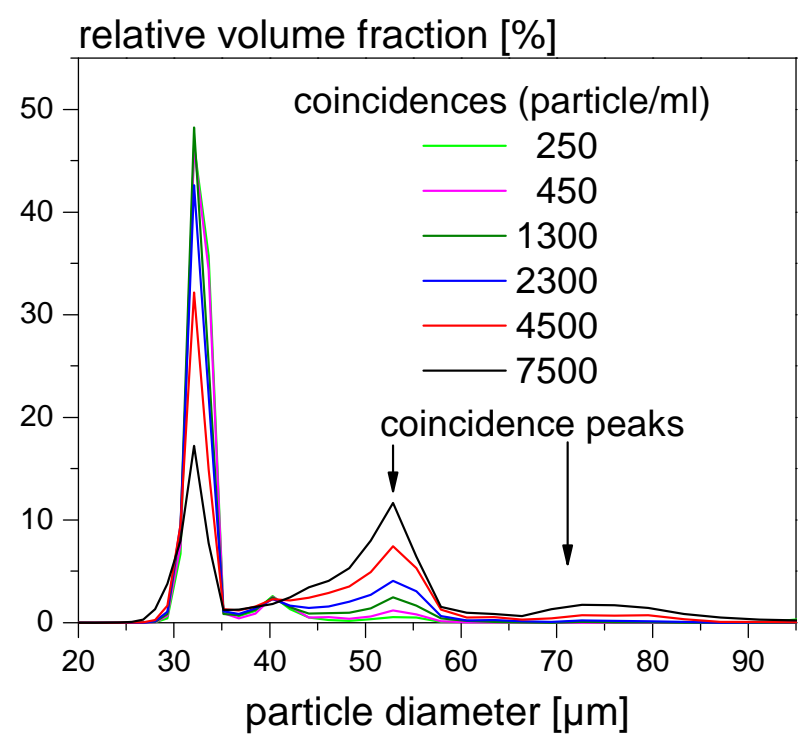

Figure 13.Effect of coincidences on the particle size distribution of size-certified polystyrene standards $(29 \mu \mathrm{m})$ measured by AccuSizer $780 \mathrm{~A}$

Figure 13 shows the particle size distribution of polystyrene standard (BS-partikel, Germany), certified for mean diameter of $29 \mu \mathrm{m}$, at different concentrations. These measurements have been performed in the extinction mode and the particle concentration has been varied adding different amount of the initial suspension to the instrument vessel, filled with $80 \mathrm{ml}$ pre-filtered and degassed distilled water. Furthermore, we used gravity drain operation in order to count all the particles within the detection volume. It can be seen from Figure 13 that the primary peaks are detected at particle size $(31.5 \mu \mathrm{m})$ slightly larger than the certificated diameter. Measurements of smaller and larger polystyrene standards (in the range of $1 \mu \mathrm{m}-78 \mu \mathrm{m}$ ) revealed that the average size detected by the Accusized is within 14\% lager than the expected size (Appendix 6.1.2). Sensor calibration issue is most probably the reason for the sizing differences despite the current recalibration performed by the AccuSizer service team (ANASYSTA e.K., Germany). The agreement of the sizing results could be probably improved by increasing the number of calibration standards in the size area of interest.

The secondary peaks in Figure 13 occur at approximately 1.7 times and 2.5 times the sphere diameter and increase with increasing the sample concentration (accordingly coincidence rate). Furthermore it can be seen that the relative volume fraction of primary particles decreases with increasing the secondary peak, indicating that part of the primary particles overlap in the detection plane and have been detected as a single large particle. These results show that the maximum coincidence rate is about 1000 particles/ml which is far below the coincidence limit indicated by the manufacture (75009000 particles/ml) [74]. 


\subsection{Volume recovery ratio}

Table 3 shows the volume recovery ratio (measured volume to the expected volume) obtained for the size-certified polystyrene standards. The volume of the detected particles has been calculated for different coincidence rates on the basis of the measurements shown in Figure 13. The expected volume was determined based on the measured particle number concentration assuming measured particle diameter $(32 \mu \mathrm{m})$. Note that this sample is not certified as a particle count standard and therefore the particle number concentration has been determined experimentally by the following procedure: defined amount of the particle size standard was repeatedly measured at coincidence level 1000 particle/ml, subsequently the number of particle counts detected within the primary peak area were averaged out of several reproducible experiments. It has been found that the number concentration of the measured monodispersed particles is about $1.62 \cdot 10^{6}$ particles $/ \mathrm{ml}$, which is in the range indicated by the manufacturer.

Table 3. Volume recovery results obtained by AccuSizer 780A for the size-certified polystyrene standard. Expected volume was calculated assuming measured particle diameter $(32 \mu \mathrm{m})$

\begin{tabular}{lccc}
\hline $\begin{array}{l}\text { Coincidences } \\
\text { (particle/ml) }\end{array}$ & $\begin{array}{c}\text { Expected volume } \\
\left(\mathbf{1 0}^{-\mathbf{4}} \mathbf{~ m l}\right)\end{array}$ & $\begin{array}{c}\text { Measured volume } \\
\left(\mathbf{1 0}^{-4} \mathbf{~ m l}\right)\end{array}$ & $\begin{array}{c}\text { Volume recovery } \\
\text { ratio }\end{array}$ \\
\hline 250 & $1.39 \pm 0.1$ & $1.7 \pm 0.7$ & 1.22 \\
450 & $2.78 \pm 0.1$ & $2.8 \pm 0.2$ & 1.01 \\
1300 & $6.95 \pm 0.1$ & $7.2 \pm 0.2$ & 1.04 \\
2300 & $13.9 \pm 0.1$ & $16.6 \pm 0.2$ & 1.19 \\
4500 & $27.8 \pm 0.1$ & $39 \pm 0.2$ & 1.44 \\
7500 & $55.6 \pm 0.1$ & $82 \pm 0.2$ & 1.47 \\
\hline
\end{tabular}

It can be seen in Table 3 that the measured volume is in agreement with expected volume for count rates in the range between about 500 particles $/ \mathrm{ml}$ and 1500 particles $/ \mathrm{ml}$. Increasing the coincidence rate further the volume recovery ratio increases with increasing the coincidence rate. As evidenced in Figure 13, this is due to the false counting of overlapping primary particles as a single large particle. At the lowest count rate (250 particles/ml) large fluctuations in the recovery ratio have been observed. This can be explained by fluctuations of the particle concentration in the injection volume. Sedimentation of the polystyrene standard particles contributes to inhomogeneities in the initial suspension which leads to an increase of the particle concentration fluctuations with decreasing the pipette delivery volume. It should be also mentioned that counts in the smallest size channels $(2-6 \mu \mathrm{m})$, that are most affected by the background noise (air bubbles and contaminations), were excluded from the analysis. However, the additive 
effect of background counts contributed only by less than $1 \%$ to the measured volume of the polystyrene standard used in this study.

\subsection{Analysis of micron-sized particles in nanodispersions}

The aim of the present study is to use the SPOS technique to analyse small amount of large particles in nano-dispersions. Therefore it was examined, whether the polystyrene standard particles are detectable in the background of nano-particles. For this purpose small amount of the polystyrene standard was mixed in the instrument vessel with polyurethane dispersion containing primarily nano-particles. Measurements at different coincidence rates were performed similarly to the experiments described above. No influence of the polyurethane nano-particles on the volume recovery ratio of the polystyrene micron-sized particles was detected and the results superimpose the data reported in Table 3.

Comparison between the particle size distributions of fine pre-filtered ( $1 \mu \mathrm{m}$ mesh size) polyurethane (PU) suspension, polystyrene standard and a mixture of both is given in Figure 14. For these measurements the coincidence rate was set to 1000 particles $/ \mathrm{ml}$. The peak at about $10 \mu \mathrm{m}$ in the size distribution of the fine pre-filtered polyurethane dispersion is due to small amount ( $80 \mathrm{ppm})$ of pre-existing micron-sized objects (see section 3.2.1.1). The particle size distribution of the polystyrene standard almost superimposes the one of the mixture with polyurethane, despite the large amount of nanoparticles. The amount of polystyrene particles is about $5 \mathrm{vol} \%$ of the polyurethane particles.

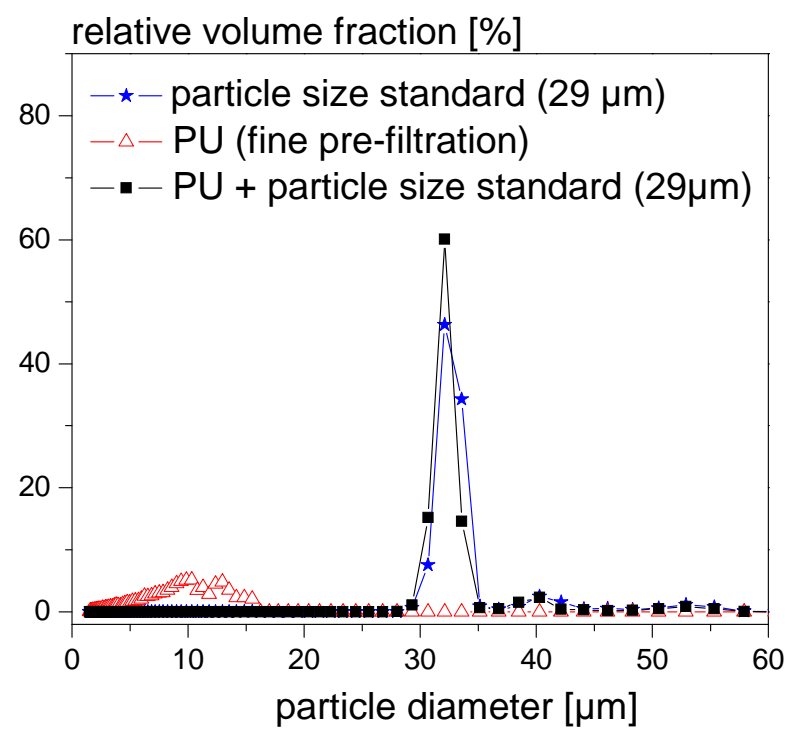

Figure 14. Comparison between the particle size distribution of size-certified polystyrene standards $(29 \mu \mathrm{m})$, fine pre-filtered PU suspension and a mixture of both

In summary, these results reveal that the SPOS method is sensitive to small concentration of micron-sized particles and the large amount of particles below the measuring 
range of the instrument does not influences neither the particle size distribution nor the volume recovery ratio of coarse particles in the micrometer range. Hence the SPOS technique can be used to determine the amount and size of micron-sized particles in nano-particle dispersions. On the other hand, care should be taken to avoid coincidences and background counts to increase the accuracy of the SPOS technique.

\subsubsection{Shear cell}

Shear flow aggregation experiments were performed using a rotational rheometer RS150 (ThermoHaake, Germany) equipped with a double concentric cylinder shear cell (DIN 53544). The inner and outer radii of the stator are $17.75 \mathrm{~mm}$ and $21.7 \mathrm{~mm}$, respectively, while the values for the rotor are $18 \mathrm{~mm}$ and $21.4 \mathrm{~mm}$. This results in an inner and outer gap width of $0.275 \mathrm{~mm}$. The shear rate employed was in the range of 10-8000 $\mathrm{s}^{-1}$. For determination of agglomerate size, the samples taken after the shear experiment were gently withdrawn from the tank into the AccuSizer container using a pipette. The same procedure was used with the samples after the ring-slit experiments in order to determine the size distribution and volume fraction of aggregates after experiments at different flow rates

\subsection{Materials}

This section presents experimental results characterizing the latex dispersions used as model colloids in this study. The surfactants used for colloidal stabilization and monodispersed polymethyl methacrylate (PMMA) particles will be briefly described.

\subsubsection{Highly concentrated polymer dispersions}

Three highly concentrated aqueous latex dispersions are used as model colloids. The characteristics of the investigated samples are summarized in Table 4. The polyurethane and polychloroprene dispersion were supplied by Bayer MaterialScience as raw materials. A polystyrene dispersion was provided by Dow Chemical Company. The suspensions investigated here are designed for a shelf-life of more than six months. According to the manufacturer, the polyurethane dispersion is synthesized by solution polymerization and subsequent precipitation without the use of surfactants [75]. The polyurethane particles are electrostatically stabilized by surface charges due to ionized sulfonate groups. In the case of the polychloroprene particles the surface charge arises from adsorbed surfactant molecules with carboxylic groups (abietic acid). Information about the chemical nature of the surface charge of the commercial polystyrene particles is not available. 
Table 4. Properties of the investigated dispersions

\begin{tabular}{lccc}
\hline Property & Polychloroprene & Polyurethane & Polystyrene \\
\hline Volume fraction / vol.\% & 50 & 40 & 47 \\
Glass transition temperature $/{ }^{\circ} \mathrm{C}$ & -40 & -40 & 95 \\
Mean particle diameter $/ \mathrm{nm}$ & 100 & 137 & 140 \\
$\mathrm{pH}$ & 12 & 7 & 6 \\
$\kappa^{-1} / \mathrm{nm}$ & 1 & 10 & 1 \\
Zeta-potential / mV & -62 & -56 & -59 \\
\hline
\end{tabular}

Note, that the particle volume fraction $(\phi)$ was calculated by relating the solid content, $c_{s}$; to the density of the particles $\rho$ and the continuous phase $\rho_{\mathrm{fl}}$ as follows:

$$
\phi=\frac{c_{s} \rho_{f l}}{c_{s} \rho_{f l}+\left(1-c_{s}\right) \rho}
$$

The solid content was measured using halogen moisture analyzer (HR83, Mettler Toledo, Germany). and the density of the polycloroprene, polyurethane and polystyrene particles was set to equal $1.21 \mathrm{~g} / \mathrm{cm}^{3}, 1.15 \mathrm{~g} / \mathrm{cm}^{3}$ and $1.05 \mathrm{~g} / \mathrm{cm}^{3}$ respectively.

\subsubsection{Particle and aggregate size distribution}

Photon correlation spectroscopy (PCS, N4 plus, Beckman Coulter Inc., Germany) was used to determine the size distribution of the particles. The measuring principle is described elsewhere [76]. Figure 15 shows the volume-weighted particle size distribution of the investigated suspensions. The volume weighted mean diameter of the particles is about $100 \mathrm{~nm}$ in the case of the polychloroprene particles and $140 \mathrm{~nm}$ in the case of the polyurethane (PU) and polystyrene (PS) particles. The electron micrograph (Figure 16) of the PS particles shows that the particles are spherical and confirm the mean particle size measured by the PCS instrument. Due to the low glass transition temperature (Table 4) of the polychloroprene and PU particles, they are soft and deformable at room temperature and could not be seen by electron microscopy. 


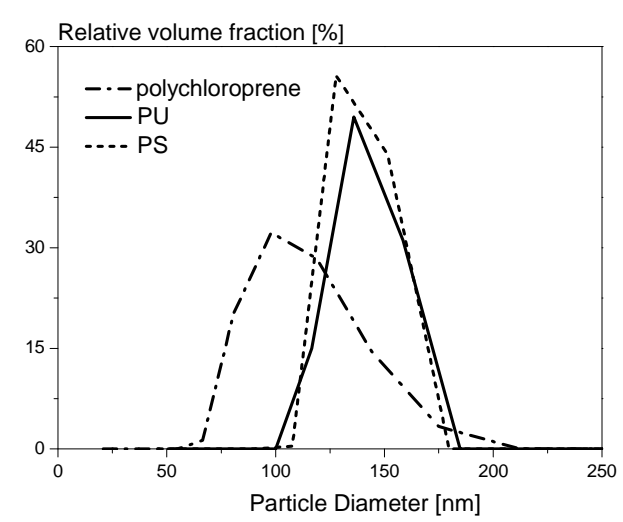

Figure 15. Volume-weighted particle size distribution of the investigated PU, PS and polychloroprene suspensions measured by PCS

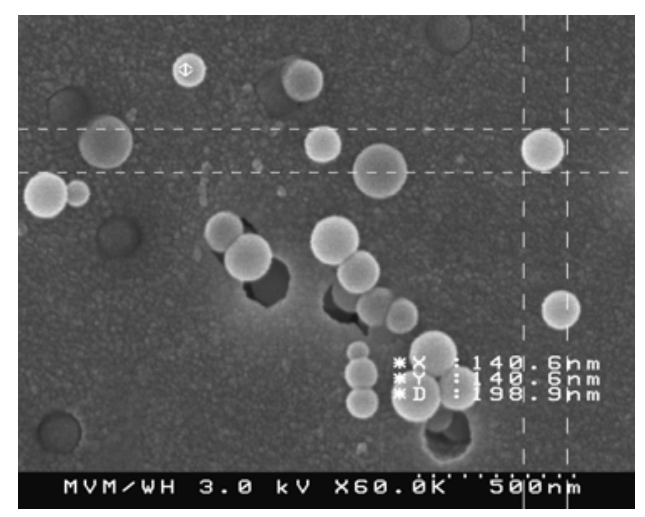

Figure 16. Electron micrograph of the PS particles

The polymerization process during manufacturing of latex dispersions often involves formation of aggregates or particles larger than the primary particles. These impurities account only for a small fraction of the total particle content in the order of $100 \mathrm{ppm}$. These large particles or agglomerates, which are almost unavoidable in technical manufacturing processes for nano-particle suspensions, play a crucial role in flow-induced aggregation, as will be shown below. Therefore, we have characterized this fraction carefully as described on the following pages.

Light microscopy observations (Axiovert 200 Inverted Fluorescence Microscop, Carl Zeiss, Germany) revealed that the PU and PS dispersions contain predominantly elongated and irregular agglomerates, in contrast to the polychloroprene dispersion that contains smaller but mainly spherical micron-sized particles (Figure 17). It should be noted that we use the term micron-sized particles for all objects larger than the primary particles without knowledge of their origin or composition. 

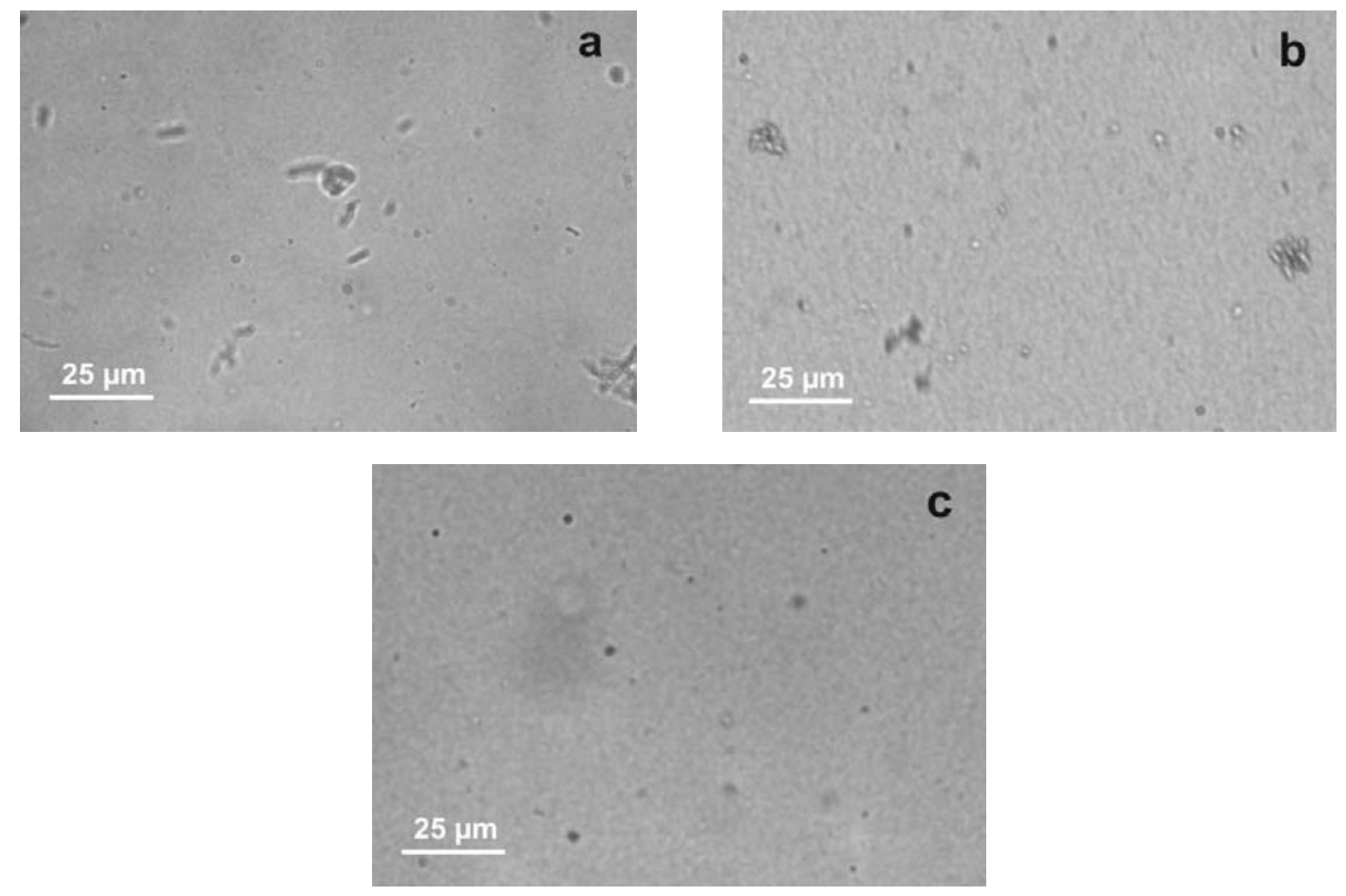

Figure 17. Light microscopy image showing aggregates in coarse pre-filtered PU- (a), PS- (b) and polychloroprene dispersion (c)

Dispersions were always pre-filtered prior to ring-slit experiments using synthetic woven monofilament fabrics purchased from Sefar AG with nominal mesh size $1 \mu \mathrm{m}$ (Sefar PETEX) and $25 \mu \mathrm{m}$ (Sefar NITEX). Pre-filtration was performed very gently using large filters to maximize the filtration area. It was difficult to control the filtration efficiency due to the irregular shape of the micron-sized particles in the investigated dispersions. Thus, in this paper, the filter with $1 \mu \mathrm{m}$ mesh size is referred to as a "fine filter" and correspondingly the "25 $\mu \mathrm{m}$ filter" as a "coarse filter".

The PCS method is convenient to determine the mean value and wigth of a particle size distribution, however a small amount of coarse particles and aggregates in the micrometer range cannot be detected. Thus the quantitative characterization of pre-existing aggregates with respect to filtration was provided by SPOS measurements as described in section 3.1.3. Figure 18a shows the cumulative volume distribution results for the PS dispersion and Figure $18 \mathrm{~b}$ for the PU and polychloroprene dispersion. These data quantitatively confirm that the micron-sized particles in the coarse pre-filtered PU and PS dispersions have a median equivalent diameter $\left(\mathrm{d}_{50}\right)$ of $9 \mu \mathrm{m}$ and $6 \mu \mathrm{m}$ respectively, whereas the micron-sized particles in the polychloroprene dispersion are smaller $\left(\mathrm{d}_{50}=1.5 \mu \mathrm{m}\right)$. The effect of pre-filtration is evident for the PU and PS dispersions but it has no significant effect on the polychloroprene dispersion. 

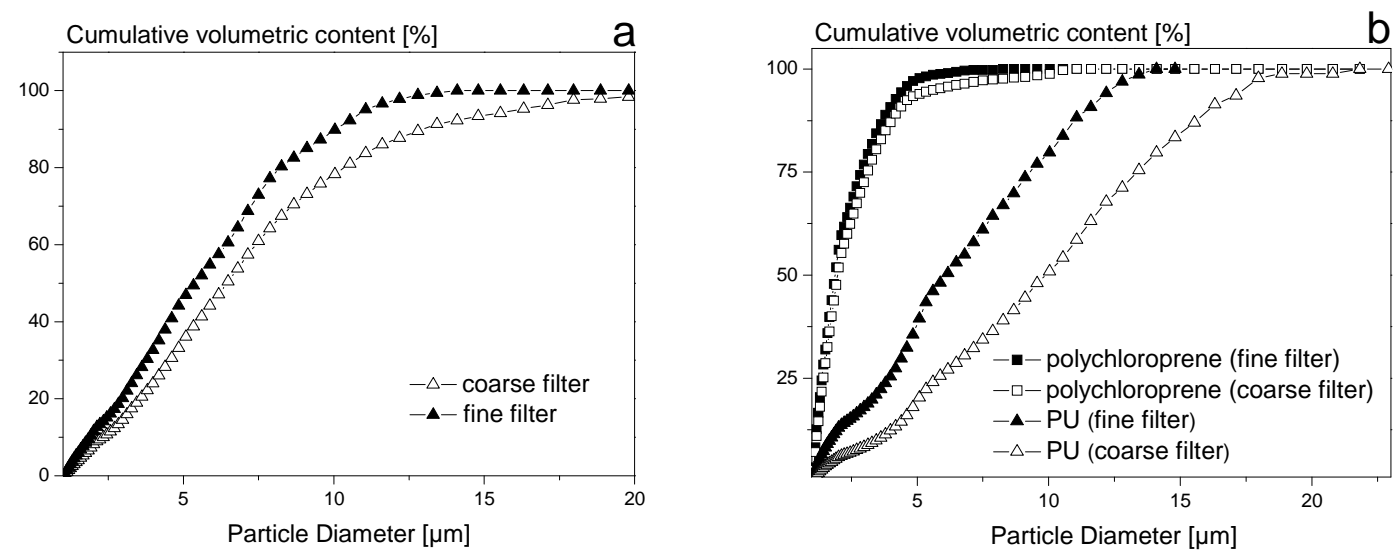

Figure 18. Cumulative volumetric distribution of micron-sized particles in PS (a) and PU and polychloroprene (b) from the measurements using AccuSizer 780A.

It should be noted that the measured diameter in the case of PU and PS is rather overestimated due to both the calibration issue (see section 3.1.3.2.1 and appendix 6.1.2) and the irregular, predominantly elongated shape of micron-sized particles. The laminar flow through the optical sensor causes the longest particle dimension to be aligned with the direction of the flow, thus the observed particle size is related to a projected area containing the long axis and is displayed as an equivalent sphere diameter.

Table 5. Quantitative analysis of aggregate fractions in the investigated PU, PS and polychloroprene dispersions

\begin{tabular}{|c|c|c|c|c|c|c|}
\hline \multirow{2}{*}{ Filtration size } & \multicolumn{2}{|c|}{ PU } & \multicolumn{2}{|c|}{ polychloroprene } & \multicolumn{2}{|c|}{ PS } \\
\hline & coarse & fine & coarse & fine & coarse & fine \\
\hline $\begin{array}{l}\text { volume fraction of aggre- } \\
\text { gates [ppm] }\end{array}$ & 204 & 80 & 47 & 37 & 365 & 240 \\
\hline$\left(\mathrm{V}_{\text {aggregates }} / \mathrm{V}_{\text {dispersion }}\right)$ & & & & & & \\
\hline $\begin{array}{l}\text { number of aggregates } \geq 1 \mu \mathrm{m} \\
\left(10^{6} \text { aggregates } / \mathrm{ml}_{\text {dispersion }}\right)\end{array}$ & 4.6 & 4.2 & 16 & 14 & 24 & 22 \\
\hline
\end{tabular}

The SPOS technique was also used to determine the volume fraction of the measured aggregates with respect to the filtration efficiency of the different filters. The results are reported in Table 5. The effect of filtration is again evident for the PU and PS suspension. It can be also seen that the highest volumetric concentration of micron-sized particles is present in the PS suspension.

\subsubsection{Electrochemical properties}

The electrical properties of particles in aqueous suspensions generally depend on the $\mathrm{pH}$ and ionic strength of dispersion medium and can be characterized by the zeta-potential. 
The ionic strength of the PU and polychloroprene suspensions was determined by conductivity measurements (Conductivity meter Cond 340i, WTW, Germany) of the suspension serum compared with the conductivity of reference $\mathrm{KCl}$ solutions of known salt concentration. The $\mathrm{KCl}$ salt has been chosen since it completely dissociates in water and similarly as the salts dissolved in the commercial PU and polychloroprene suspension, is a monovalent salt. As expected the measured conductivity of $\mathrm{KCl}$ solutions increases linearly with increasing salt concentration.

The suspension serum has been obtained by ultracentrifugation of suspended nanoparticles using an analytical ultracentrifuge Optima XL-I (Beckman Coulter). The ultracentrifugation has been performed at Bayer MaterialScience. The results of measured suspension and serum conductivity and the correspondent ionic strengths are presented in

Table 6. It can be seen that the conductivity of the PU suspension is higher than the conductivity of the serum, while in the case of polychloroprene, suspension conductivity is lower than the serum conductivity. This can be explained with the effect of the diffuse charge cloud surrounding the particles. According to the Maxwell theory [77] the conductivity of suspension can be expressed as follows:

$$
\sigma=\sigma_{e} \frac{1+2 X \phi}{1-X \phi}
$$

where $\sigma$ and $\sigma_{\mathrm{e}}$ are respectively suspension and serum conductivity and $X$ is defined as:

$$
X=\frac{\sigma_{p}-\sigma_{e}}{\sigma_{p}+2 \sigma_{e}}
$$

with $\sigma_{\mathrm{p}}$ representing the particle conductivity arising from transport processes in the double layer. Hence, when $\sigma_{\mathrm{p}}$ is higher than $\sigma_{\mathrm{e}}$, the suspension conductivity, $\sigma$, is expected to be higher than the serum conductivity, $\sigma_{e}$, and $\sigma$ increases with increasing the particle volume fraction, $\phi,[78]$. On the other hand, if $\sigma_{p}$ is smaller than $\sigma_{e}$, the suspension conductivity is smaller than the conductivity of suspending medium. In the case of non-conducting uncharged particles, the particles act as obstacles to the ion flux and conductivity is diminished relative to dispersion medium.

For the polychloroprene and PU suspension the ionic strength is determined to be about $78 \mathrm{mM}$ and $8 \mathrm{mM}$ respectively. In the case of the PS suspension the serum conductivity has not been analyzed and information about the valency of dissolved salts is not available. However the measured suspension conductivity suggests relativly high ionic strength that is most probably in the same range as for the polychloroprene suspension. 
Table 6 Electrical conductivity and ionic strength of investigated suspensions

\begin{tabular}{lccc}
\hline Property & Polychloroprene & PU & PS \\
\hline dispersion conductivity & $7.9\left(19^{\circ} \mathrm{C}\right)$ & $1.21\left(20^{\circ} \mathrm{C}\right)$ & $8.18\left(25^{\circ} \mathrm{C}\right)$ \\
{$[\mathrm{mS} / \mathrm{cm}]$} & & & \\
serum conductivity & $9\left(19^{\circ} \mathrm{C}\right)$ & $0.95\left(20^{\circ} \mathrm{C}\right)$ & - \\
{$[\mathrm{mS} / \mathrm{cm}]$} & & & - \\
ionic strength & $78 \pm 5$ & $8 \pm 1$ & \\
{$[\mathrm{mM}]$} & & & \\
\hline
\end{tabular}

The $\mathrm{pH}$ values of investigated suspensions are listed in Table 4 and have been measured with pH-meter (Portamess 911pH, Knick Elektronische Meßgeräte, Germany) equipped with a glass $\mathrm{pH}$ sensor (SE 102). The $\mathrm{pH}$ value may strongly influence the dissociation behavior of surface groups and hence the surface charge density. The $\mathrm{pH}$ influence on the surface charge density can be detected by zeta-potential measurements.

The zeta-potentials of investigated suspensions are measured with a Zetasizer Nano (Malvern) at particle concentration below $0.1 \%$ by volume. Dispersions have been diluted with $\mathrm{KCl}$ solutions with the same ionic strength and $\mathrm{pH}$ as the initial suspensions, in order to keep the particle electrical properties unchanged. The zeta-potentials of investigated samples are reported in Table 4 . They are all strongly negative indicating elestrostatically well stabilized dispersions. The zeta-potentials of the PU and polychloroprene suspensions are shown as a function of $\mathrm{pH}$ in Figure 19. The zeta-potential of the PU suspension is largely independent of $\mathrm{pH}$, since the sulfonate surface groups are ionized over the investigated $\mathrm{pH}$ range and maintain a strong $\mathrm{pH}$ independent negative charge. In the case of the polychloroprene suspension, the magnitude of the zetapotential decreases with decreasing the $\mathrm{pH}$ value. As mentioned in section 3.2.1, the surface charge in this case arises from adsorbed abietic acid molecules $\left(\mathrm{pK}_{\mathrm{a}} \approx 7\right)$ whose protonation begins already at alkaline $\mathrm{pH}[79]$. 


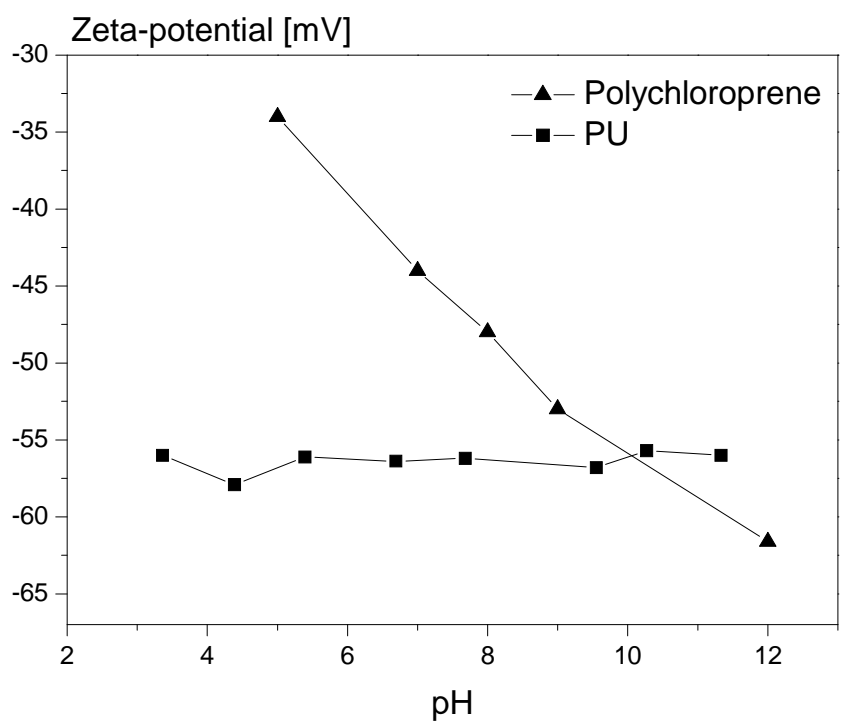

Figure 19. Zeta-potential as a function of the $p H$ value for the polychloroprene and PU suspension at ionic strength of $80 \mathrm{mM}$ and $8 \mathrm{mM}$ respectively

\subsection{Procedure for preparing dispersions of known ionic strength}

Most appropriate procedure for variation of the ionic strength of concentrated dispersions is dialysis against electrolyte solution of known ionic strength. In principle, the increase of the ionic strength can be achieved by adding electrolyte directly to the dispersion but this result in an undesirable dilution, as the electrolyte should be in the form of relatively dilute solution to prevent local coagulation of the particles.

In this study, the PU dispersion has been dialyzed against $\mathrm{KCl}$ solution of $50 \mathrm{mM}$ ionic strength, which is higher than the ionic strength in the initial dispersion $(8 \mathrm{mM})$. The dispersion was sealed in small diameter $(45 \mathrm{~mm})$ ready-to-use dialysis tubings (Spectra/Pro 2, Spectrum Laboratories Inc., Netherlands) with approximate molecular weight cut off 12000-14000 Daltons. The dialysis tubings have been immersed in $\mathrm{KCl}$ solution, such that the $\mathrm{KCl}$ solution is of large excess, at least 10:1 by volume. The conductivity of the $\mathrm{KCl}$ bath has been monitored and the solution has been replaced daily. The dialysis can be considered completed when the electrical conductivity of the bath solution does not change over time. This usually requires several days, as the dialysis process is faster at the beginning but then slows down due to the decrease of the concentration gradient. Upon completion, the dispersion was stored in glass bottles and the flow stability was measured within one week.

It should be mentioned that dialysis of a dispersion against salt solution of lower ionic strength requires an external pressure to be applied the dialysis tubing in order to counterbalance the osmotic pressure and prevent undesirable dilution of the dispersion and eventual rupture of the membrane. This can be achieved by placing weights on the dialysis tubings containing the dispersion, which need to be removed periodically and the dispersion gently shaken to minimize coagulum build-up. 


\subsubsection{Identification of the stabilization mechanism with high frequency rheology using torsional resonators}

The frequency dependence of the elastic modulus $G_{\infty}^{\prime}$ in the high frequency limit was measured using two torsional resonators (IdM, University Ulm, Germany) in order to get an insight into the dispersion stabilization mechanism. The experimental setup, measuring principle and the calibration procedure are described in detail elsewhere [80]. Torsional resonance oscillation was applied at four distinct frequencies ((52, 120, 198 and 364$) \times 10^{3} \mathrm{rad} / \mathrm{s}$ ), for which the criterion for high frequency regime is fulfilled, i.e. the applied shear field is much faster than the Brownian motion of colloidal particles [81]:

$$
\frac{\omega}{2 \pi}>>\frac{D_{s}^{s}}{h^{2}} \approx \frac{D_{0}}{a^{2}}
$$

where $\omega$ is the frequency of oscillation, $h$ the mean interparticle separation, $a$ the particle radius, $\mathrm{D}_{0}$ the bare diffusion coefficient and $D_{s}^{s}$ is the short-time self diffusivity( i.e. the characteristic small displacement mobility of a particle which decreases with increasing particle concentration).

Fritz et al. [80] pointed out that the frequency dependency of the elastic modulus in the high frequency limit can be used to distinguish whether a concentrated suspension is stabilized electrostatically, sterically or it resembles a hard sphere suspension. It has been shown theoretically that the elastic modulus in the high frequency limit has a frequency independent value in the case of electrostatically stabilized dispersions. Due to the presence of near field hydrodynamic interactions [82] the elastic modulus of hard sphere suspensions exhibits $\omega^{1 / 2}$ dependence in the high frequency limit. Sterically or electrosterically stabilized particles the presence of swollen polymer chains induces a frequency dependence of $G_{\infty}^{\prime}$ with an exponent smaller than 0.5 . The increase of elastic modulus with increasing frequency is due to the relaxation of the polymer chains on particle surface.

In the frequency range investigated here the frequency dependence of $G_{\infty}^{\prime}$ can be approximated by a power-law: $G_{\infty}^{\prime}=A^{\prime} \omega^{n^{\prime}}$. For the PU suspension a high frequency plateau for the elastic modulus has been found with $n^{\prime}=0.03 \pm 0.05$ which is in agreement with the theoretical prediction and confirm that the PU suspension is purely electrostatically stabilized. Dialysis of the PU suspension against salt solution with a high salt concentration $(\mathrm{I}=50 \mathrm{mM})$ allows us to decrease the range of electrostatic repulsion so that the system resembles hard sphere behavior. In this case, a strong frequency dependence is observed $\left(n^{\prime}=0.9 \pm 0.14\right)$ which is higher than the theoretically predicted value and is attributed to systematic error in the calibration constants used for the data evaluation. 
Similarly, for the polychloroprene and polystyrene suspensions an exponent close to unity is found to describe the high frequency dependence of $G_{\infty}^{\prime}$. Despite the overestimated exponent the observed frequency dependence of $G_{\infty}^{\prime}$ confirms that the investigated latex suspensions are purely electrostatically stabilized and no polymer chains are adsorbed or grafted onto the particle surface.

\subsubsection{Rheological estimation of electrostatic double-layer thickness}

It is well known that the electrostatic interactions have a large influence on the low shear viscosity of colloidal suspensions. An estimate of the electrostatic double-layer thickness can be obtained by mapping the divergence in the zero-shear viscosity onto that of hard sphere dispersions. The volume fraction dependency of zero shear viscosity of the suspensions investigated here is well described by the Quemada equation [83]:

$$
\frac{\eta_{0}}{\mu}=\left[1-\frac{\phi}{\phi_{\max }}\right]^{-2}
$$

Where $\eta_{0}$ and $\mu$ are the zero-shear viscosity of the dispersion and viscosity of the solvent respectively and $\phi_{\max }$ is the effective maximum packing fraction (the volume fraction at which the viscosity becomes infinite). It has been shown that for colloidal suspensions the zero-shear viscosity diverges at the volume fraction of the colloidal glass transition $\phi_{\mathrm{g}}$ (i.e. $\phi_{\max }$ is equal to $\phi_{\mathrm{g}}$ ). Analogous to the glass transition of ordinary glasses which occurs at a certain temperature below which the molecular mobility vanishes, a similar phenomenon is observed in colloidal suspensions. Above a critical particle concentration, their mobility decreases to zero due to the caging effect of the neighbouring particles [84] [85]. For hard sphere suspensions $\phi_{\mathrm{g}}$ is equal to 0.58 [86] [87].

Equation (78) is often used to model both concentrated charged and hard sphere suspensions [88] [89] [90]. The zero-shear viscosities are extracted from well-defined Newtonian low-shear plateaus shown in Figure 20 for the all three dispersions investigated here. These measurements have been performed using a rotational rheometer RS-150 (ThermoHaake, Germany) equipped with a concentric cylinder shear cell (Z40/DIN 53019). 

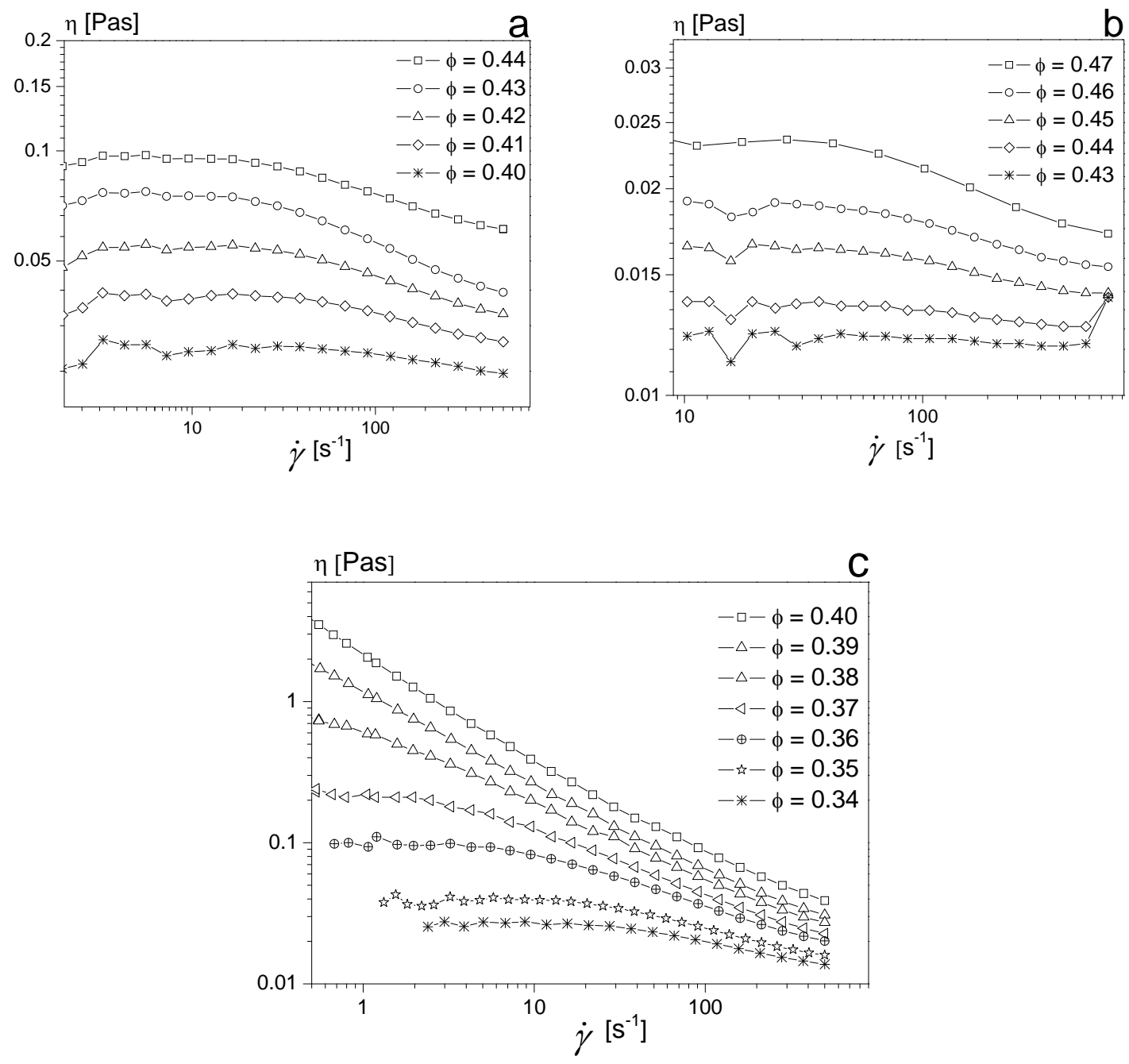

Figure 20. Dispersion viscosity as function of shear rate for the polychloroprene (a), PS (b) and $P U$ dispersion (c) at different volume fractions

The $\phi_{\max }$ values were determined from the $x$-intercept in a plot of $\left(\eta_{0} / \mu\right)^{-0.5}$ versus volume fraction $\phi$ as show in Figure 21. For the PU dispersion the maximum packing fraction $\phi_{\max }$ was 0.386 while for the polychloroprene and PS, $\phi_{\max }$ was found to be 0.55 and 0.56 respectively, which is close to the colloidal glass transition volume fraction $\phi_{\mathrm{g}}$ $=0.58$ at which viscosity of hard sphere suspensions is supposed to diverge. The effective hard-sphere radii $a_{\text {eff }}$, which accounts for the extra excluded volume $\phi_{\text {eff }}$ due to the electrostatic double layer around the particles, can be calculated using the following scaling relations:

$$
\phi_{\text {eff }}=\phi \cdot\left(\frac{a_{\text {eff }}}{a}\right)^{3}
$$




$$
\phi_{\text {eff }}=\phi \cdot\left(\frac{\phi_{\max , h s}}{\phi_{\max }}\right)
$$

where $\phi_{\max , h s}$ is the maximum packing fraction of hard sphere suspensions. For random close packing fraction, experiments of hard sphere suspensions have shown that $\phi_{\max , h s}$ is in the range 0.58-0.64 [91] [86]. We take $\phi_{\text {max }, h s}=0.58$, the value that corresponds to the colloidal glass transition, to calculate the value of $a_{\text {eff. Note that equation (79) is }}$ based on the assumption of monodispersed particles and the calculated values of the Debye length $\kappa^{-1}$, listed in Table 4 , are a crude estimate of the range of repulsive interactions.

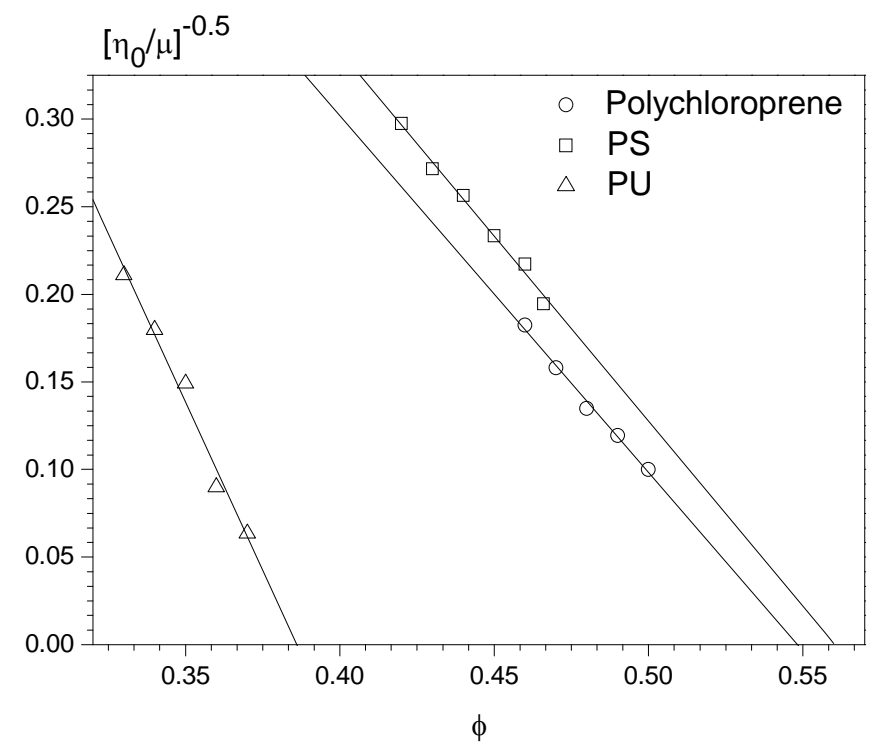

Figure 21. Determination of $\phi_{\max }$ from zero shear viscosity at different volume fractions (Figure 20)

\subsubsection{Monodispersed PMMA particles}

Monodisperse PMMA particles with diameters of 1, 10 and $14.5 \mu \mathrm{m}$ have been used in heterocoagulation experiments. The PMMA particles of $1 \mu \mathrm{m}$ diameter are electrostatically stabilized by surface charges due to ionized sulfate groups. The electrokinetic surface charge was determined to be $0.0169 \mathrm{meq} / \mathrm{g}$ by means of particle-charge detector (Mütec PCD 03), in combination with an automatic titration unit (DL 25, Mettler Toledo). The PMMA particles of $10 \mu \mathrm{m}$ and $14.5 \mu \mathrm{m}$ diameter are stabilized by polyvinylalcohol adsorbed on the particle surface, according to the manufacturer. Measurements with the particle-charge detector confirmed that these particles are not charged. These measurements have been performed at the Institute of Functional Interfaces of Campus North of the Karlsruhe Institute of Technology in the group of Professor Franzreb. 


\subsubsection{Surfactants}

In order to investigate the effect of surfactants on dispersion stability, two types of surfactant, an anionic and non-ionic surfactant, were used. The anionic sodium dodecyl sulfate (Carl Roth Ltd, Germany) has a molar mass of $288 \mathrm{~g} / \mathrm{mol}$ and provides a thin stabilizing layer owing to the small size of the SDS molecule. The SDS molecules contain 12 carbons and the length of a molecule in the stretched configuration is only about $1.7 \mathrm{~nm}$ [92]. The non-ionic polymeric surfactant, known as Thanemul FD (TANATEX Chemicals, Germany) is based on stearyl alcohol polyglycol ether and contains polymer chains of different lengths. According to the manufacture the average molar mass amounts to $1240 \mathrm{~mol} / \mathrm{g}$. 


\section{Experimental results and discussion}

\subsection{Clogging mechanism}

The purpose of this investigation is to reveal the aggregation mechanism causing the clogging of narrow gaps when concentrated dispersions are forced to flow through a sudden contraction, such as during filtration, pumping, coating or any technical operation where strong flow contraction occurs. We use the ring-slit device for this study according to the procedure described in section 3.1.1 and we focus on the effects of flow rate and particle wall interactions to elucidate the slit clogging mechanism.

\subsubsection{Effect of particle - wall interactions}

Particle deposition is one possible mechanism for clogging of microchannels by flowing nanosuspensions. The adhesive forces and corresponding deposition efficiency depend on the hydrophobic/hydrophilic interactions between particles and the wall surface. Particle deposition at the wall was examined using two slit dies with different surface properties: one plain stainless steel slit, which is hydrophilic in nature and a second one covered with a $2 \mu \mathrm{m}$ thick silicon based hydrophobic film (Toposeal, MSTagion). The results for the coarse pre-filtered PU suspension at a slit height of $21 \mu \mathrm{m}$ are presented in Figure 22 for volumetric flow rates of $314 \mathrm{~mm}^{3} / \mathrm{s}$ (Figure 22a) and $63 \mathrm{~mm}^{3} / \mathrm{s}$ (Figure $22 \mathrm{~b}$ ). We observe similar pressure increase as a function of extruded volume for the same hydrodynamic conditions. This finding suggests that the particle-wall colloidal interactions are negligible for the mechanism of slit clogging; particle deposition is insignificant for the chemical and physical conditions investigated here.
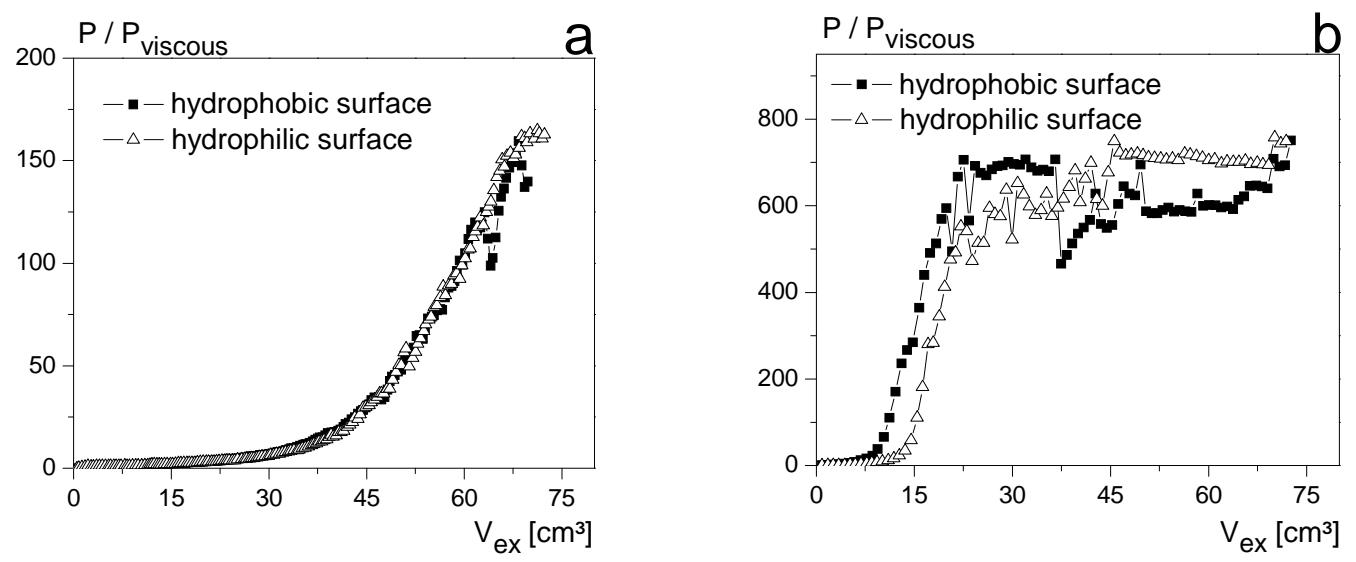

Figure 22. Effect of surface hydrophobicity on the clogging phenomenon in the ring-slit device for the PU dispersion (coarse filter, $\phi=0.4, H=21 \mu \mathrm{m}, \beta=45^{\circ}$ ) at $314 \mathrm{~mm}^{3} / \mathrm{s}$ (a) and $63 \mathrm{~mm}^{3} / \mathrm{s}$ (b) flow rate 
This is further supported by the following considerations: the stainless steel has an isoelectric point close to 5.5-6 and at higher $\mathrm{pH}$ it is negatively charged [93]. Hence, in the $\mathrm{pH}$ range relevant for the investigated dispersions, the electrostatic interactions between the negatively charged latex particles and stainless steel are repulsive and deposition on the wall is rather unlikely.

\subsubsection{Effect of flow rate}

Figure 23 shows the effect of flow rate on particle retention for three different suspensions. In all experiments, the Reynolds number for suspension flow in the slit entrance region and within the microchannel is small $(\operatorname{Re}<2.58)$. The Reynolds number in the slit $(\mathrm{H}=21 \mu \mathrm{m})$ was calculated using equation $(72$, page 34$)$, the obtained values are reported in Table 8 (page 63).
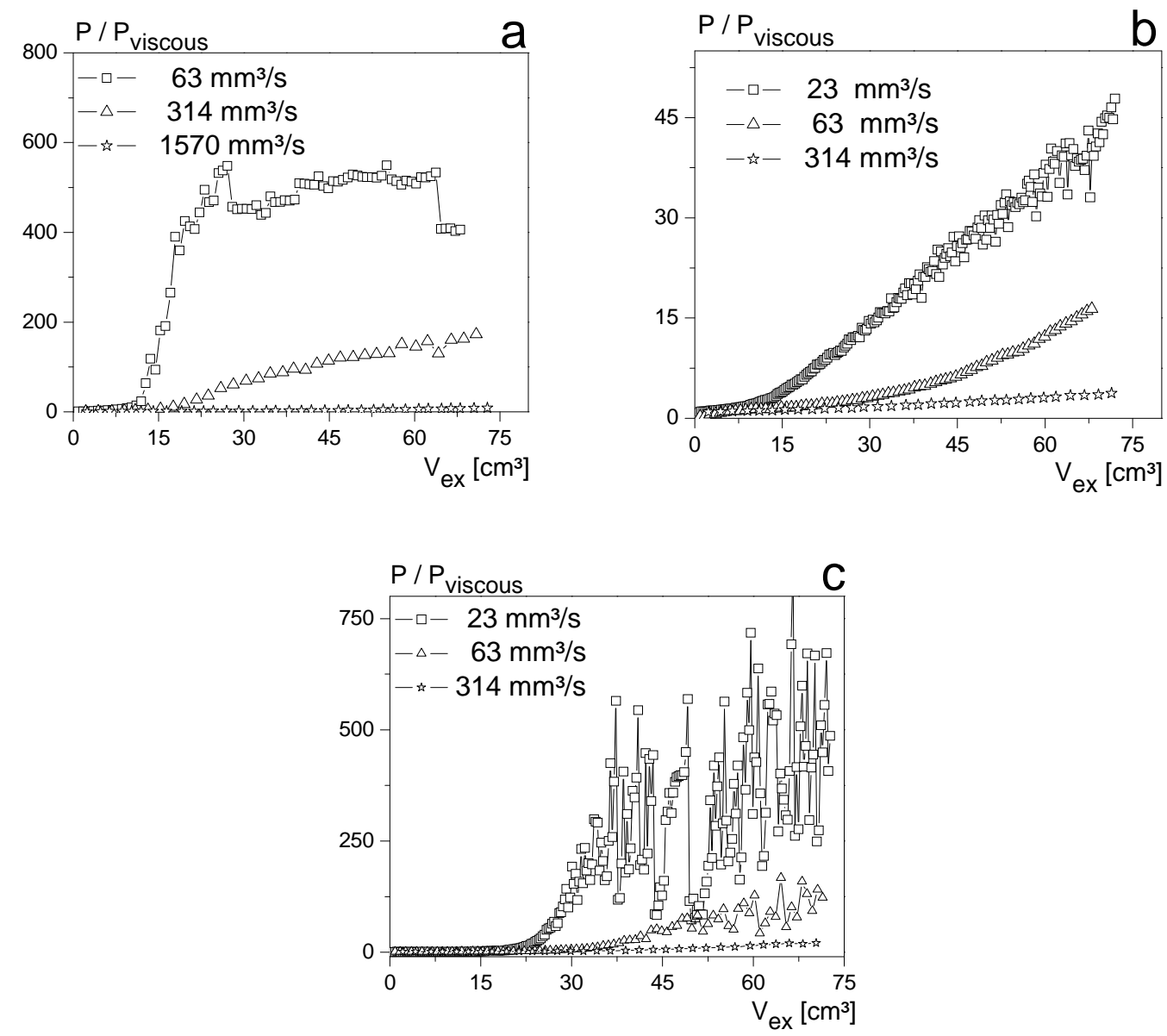

Figure 23. Effect of flow rate on the flow-induced aggregation of coarse pre-filtered PU dispersion at $\phi=0.4, H=21 \mu \mathrm{m}$ and $\beta=45^{\circ}$ (a), coarse pre-filtered polychloroprene dispersion at $\phi=$ $0.44, H=11 \mu \mathrm{m}$ and $\beta=45^{\circ}(\mathrm{b})$ and coarse pre-filtered $P S$ dispersion at $\phi=0.47, H=26 \mu \mathrm{m}$ and $\beta=30^{\circ}$ 
Results for the PU suspension (coarse pre-filtration), obtained at a slit height of $21 \mu \mathrm{m}$ are shown in Figure 23a. Similar experiments were performed with the polychloroprene dispersion but the gap height was set to $11 \mu \mathrm{m}$. (Figure 23b). In both cases the experiments were done using a slit die with a $45^{\circ}$ entrance angle. The effect of flow rate on the stability of the polystyrene dispersion (coarse pre-filtration) is demonstrated in Figure $23 \mathrm{c}$. For this data, we used a ring-slit die with a $30^{\circ}$ slit entrance angle and we fixed the slit height at $26 \mu \mathrm{m}$. The results presented in Figure 23 show a strong flow rate dependence, although the absolute values of the normalized pressure are very different for the three dispersions. The pressure increase is most pronounced when the samples are extruded at the lowest piston speed. A continuous decrease of the normalized pressure drop is detected when increasing the flow rate. These results show that we indeed observe a flow-induced phenomenon; simply collecting pre-existing objects larger than the gap height would only depend on the total amount of extruded volume but not on the flow rate. Moreover this flow rate dependence excludes plugging by hydrodynamic bridging for which the extent of slit clogging should increase with increasing flow rate [65].

Figure 24 shows the results of repeated extrusion of the coarse pre-filtered PU dispersion at an intermediate flow rate $\left(314 \mathrm{~mm}^{3} / \mathrm{s}\right)$ and at same kinematics condition $(21 \mu \mathrm{m}$ gap height and $45^{\circ}$ slit entrance angle) as for the experiments above. It can be seen that the curves almost coincide, providing another argument against clogging by straining.

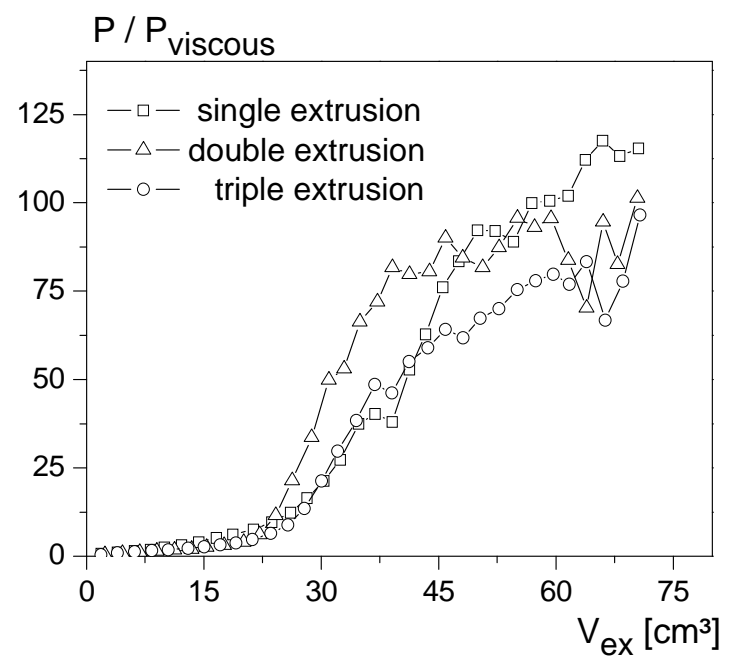

Figure 24. Effect of repeated extrusion on the clogging phenomenon for the PU dispersion (coarse filter, $\phi=0.4, H=21 \mu \mathrm{m}, \beta=45^{\circ}$ and flow rate $=314 \mathrm{~mm} / \mathrm{s}$ )

The extruded volume of the coarse pre-filtered PU suspension has been analyzed using the SPOS technique. The relative amount of aggregates found at different flow rates is given in Table 7. The amount of aggregates in the extruded sample decreases with increasing flow rate. In fact, at the lowest flow rate, a considerable increase in the agglomerate volume fraction in comparison to the sample before the ring slit test is found, 
which reveals that not all the aggregates formed during flow are collected at the gap entrance. On the other hand at the highest flow rate we detected a decrease in the aggregate volume fraction, which can be attributed to the breakup of pre-existing aggregates at these particular hydrodynamic conditions. At the intermediate flow rates the volume fraction of aggregates in the extruded dispersion remains almost unchanged. These results support the notion that the observed clogging phenomenon is a result of flowinduced aggregation, which is most pronounced at the lowest flow rates investigated here.

Table 7. Quantitative analysis of aggregates in the extruded PU dispersion (coarse pre-filtered) at different flow rates $\left(H=21 \mu \mathrm{m}\right.$ and $\left.\alpha=45^{\circ}\right)$

\begin{tabular}{ll}
\hline Flow rate $\left[\mathbf{m m}^{3} / \mathbf{s}\right]$ & Volume fraction of aggregates $\left(\mathbf{V}_{\text {aggregates }} / \mathbf{V}_{\text {dispersion }}\right)[\mathbf{p p m}]$ \\
\hline unsheared & $204 \pm 20$ \\
23 & 340 \\
63 & 216 \\
314 & 196 \\
1570 & 160 \\
\hline
\end{tabular}

\subsubsection{Particle inertia}

As discussed in section 2.5, particle's inertia can cause particle trajectories to cross the fluid streamlines and impact the wall. Plugging of narrow channels by inertial impaction has been studied by Kao et al. [94] by considering the effect of hydrodynamic interaction of the particles with the entrance geometry of the pores of nuclepore type filter. They demonstrated that in the low Stokes number range $(\mathrm{St}<0.1)$, particle inertia is insignificant and inertial impaction of particles onto the pore edges is impossible. The Stokes number in our experiments was calculated for the case of the PU suspension extruded through $21 \mu \mathrm{m}$ slit at different flow rates, using equation (71). The particle size was set to equal the median diameter of micron-sized particles $\mathrm{d}_{50}=9 \mu \mathrm{m}$ (Figure 18) in order to consider the effect large particles. As shown in Table 8, the maximum value of the Stokes number is about 0.1 , hence, inertial impaction of particles onto the slit wall at the constriction is very unlikely. The values of the Stokes number in the case of the polychloroprene and PS particles are even smaller than the values reported in Table 8 due to smaller micron-sized objects in the polychloroprene suspension and lower particle density in the case of the PS. 
Table 8. Reynolds and Stokes numbers at different flow rates in the case of $21 \mu \mathrm{m}$ gap. The particle size was set to equal the median diameter of micron-sized particles ( $9 \mu \mathrm{m})$, particle density $\rho=1150 \mathrm{~kg} / \mathrm{m}^{3}$ and suspension viscosity $\eta=0.01 \mathrm{~Pa} \cdot \mathrm{s}$, for this calculations.

\begin{tabular}{lll}
\hline Flow rate $\left[\mathrm{mm}^{3} / \mathbf{s}\right]$ & Reynolds number & Stokes number \\
\hline 23 & 0.04 & 0.002 \\
63 & 0.11 & 0.005 \\
314 & 0.52 & 0.024 \\
1570 & 2.58 & 0.12 \\
\hline
\end{tabular}

\subsubsection{Shear flow experiments}

Since flocculation of particles within the slit channel is also possible, we tested dispersion stability in a pure shear flow using a concentric cylinder shear cell. The samples were sheared for 15 minutes at different shear rates between $10 \mathrm{~s}^{-1}$ and $8000 \mathrm{~s}^{-1}$. During these experiments, the suspensions are exposed to the shear field several orders of magnitude longer than in the ring-slit experiments, but no significant increase in the number or size of aggregates due to shear could be observed. The measured volume fraction of aggregates in the PU dispersion even decreased by about $25 \%$ for shear rates higher than $100 \mathrm{~s}^{-1}$. It should be noted that the shear rate in the slit channel at the lowest piston speed applied in our experiments corresponds to $6700 \mathrm{~s}^{-1}$, which is comparable to the shear rate applied in the shear cell. Hence, aggregate formation in the simple shear flow within the slit channel is unlikely and is therefore not a factor in the experiments. Aggregate formation in the complex flow field at the slit entrance and subsequent retention of the aggregates larger than the slit height is most likely to be the dominant mechanism in the ring-slit test. Indeed, a large amount of coagulum is found at the slit entrance after extrusion experiments in which a marked pressure increase was observed.

\subsection{Hetero-coagulation in elongational flow}

In the materials section, we have shown (Figure 18 and Table 5) that all the dispersions investigated here contain a small amount of micron-sized particles. The impact of these objects on flow-induced aggregation will be discussed below.

\subsubsection{Effect of pre-filtration}

Figure 25a shows the effect of pre-filtration for the PU suspension at $314 \mathrm{~mm}^{3} / \mathrm{s}$ volumetric flow rate, where it is apparent that the pre-filtration has a dramatic effect on the clogging phenomenon. Similar data obtained for the PS suspension at $63 \mathrm{~mm}^{3} / \mathrm{s}$ are shown in Figure 25b. The trend is the same for lower as well as higher flow rates (see appendix 6.1.3). These results show that the amount and size of impurities strongly in- 
fluences the clogging mechanism. We should also mention that the polychloroprene dispersion which contains smaller agglomerates proved to be stable for the $21 \mu \mathrm{m}$ slit over the whole flow rate range.
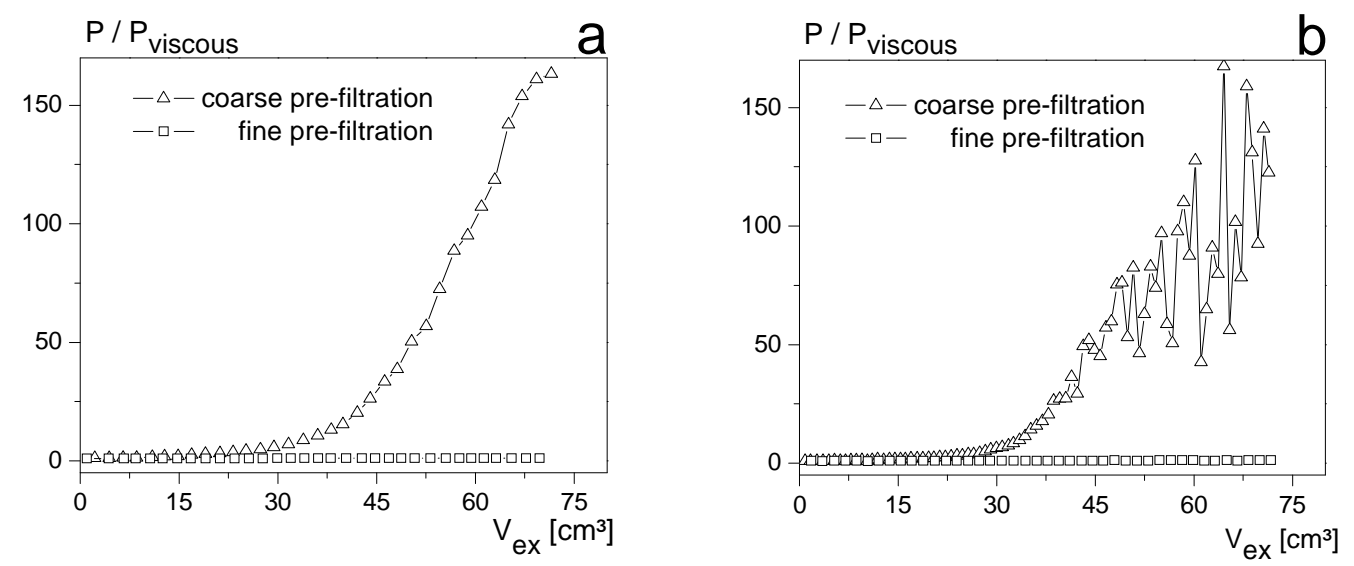

Figure 25. Effect of micron-sized particles on the flow induced aggregation of (a) PU dispersion $\left(\phi=0.4, H=21 \mu \mathrm{m}, \beta=45^{\circ}\right.$ and flow rate $\left.=314 \mathrm{~mm}^{3} / \mathrm{s}\right)$ and (b) PS dispersion $(\phi=0.47$, $H=26 \mu \mathrm{m}, \beta=30^{\circ}$ and flow rate $=63 \mathrm{~mm}^{3} / \mathrm{s}$ )

\subsubsection{Hetero-coagulation with monodispersed PMMA particles}

The irregular form of micron-sized objects strongly influences the filtration efficiency and makes it difficult to control the size and amount of aggregates. We therefore added monodisperse PMMA particles of a defined size and concentration to the fine prefiltered PU dispersion in order to systematically investigate the effect of impurities on the flow-induced aggregation. For comparison the homo-coagulation of PMMA particles in the ring-slit device was also tested by conducting experiments with PMMA particles dispersed in an aqueous glycerine solution with the same viscosity as the PU dispersion. Figure 26 shows the effect of PMMA particles on the flow-induced aggregation for the fine pre-filtered PU suspension. The data in Figure 26a refers to a particle concentration of $100 \mathrm{ppm}$ (relative to the total dispersion volume) and those in Figure 26b to $1000 \mathrm{ppm}$ particle concentration. It is evident from thise plots that the PMMA dispersion itself is stable in the converging flow field, as we observe a constant pressure signal is observed. However, a significant pressure increase is detected when the same amount of PMMA particles is dispersed in the PU suspension. The implication of these results is that slit clogging is due to the hetero-coagulation of PU and PMMA particles. 

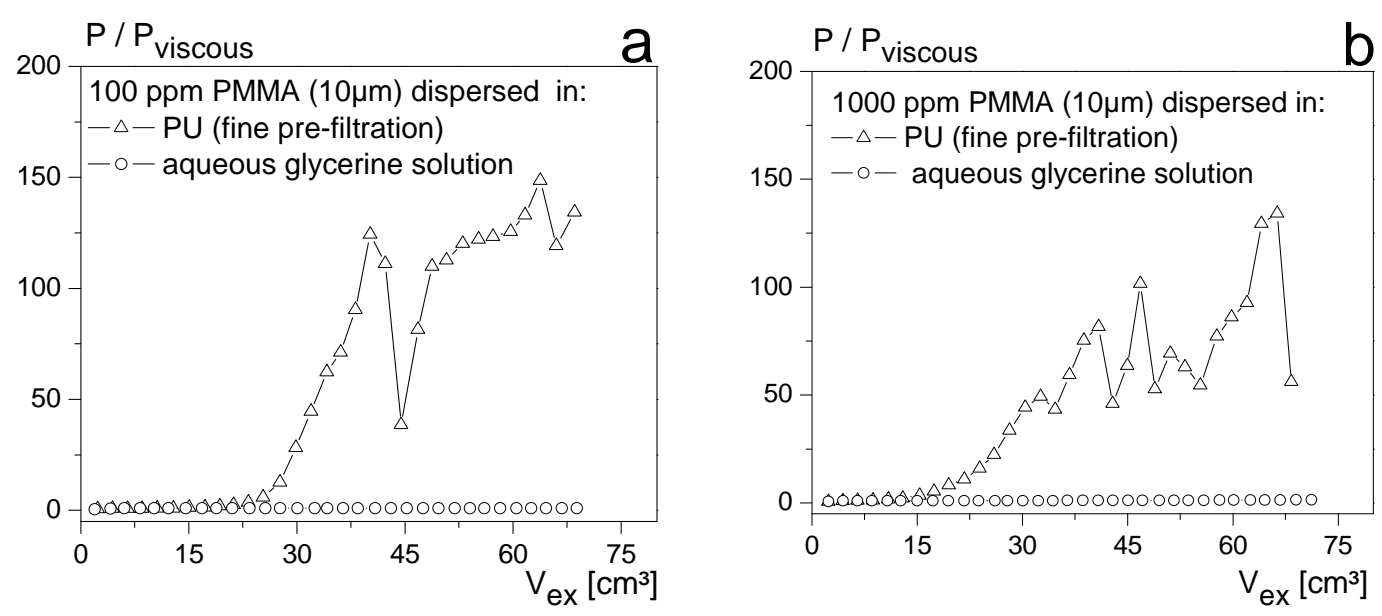

Figure 26. Effect of $10 \mu \mathrm{m} P M M A$ particles on the flow induced aggregation of $P U$ dispersion at 100 ppm PMMA (a) and at 1000 ppm PMMA particles ( $\phi=0.4, H=21 \mu \mathrm{m}, \beta=45^{\circ}$ and flow rate $\left.=314 \mathrm{~mm}^{3} / \mathrm{s}\right)$

Further evidence for hetero-coagulation is provided by electron micrographs of the coagulum retained at the slit entrance after experiments with $100 \mathrm{ppm}$ and $1000 \mathrm{ppm}$ of $10 \mu \mathrm{m}$ PMMA particles dispersed in the PU dispersion, as shown in Figure 27. The continuous, indistinguishable mass that we see on the images is created by aggregated PU particles which are soft and deformable at room temperature due to the very low glass transition temperature $\left(-40^{\circ} \mathrm{C}\right)$. In contrast to the PU particles, the PMMA particles are hard at room temperature and appear as spheres on the micrographs. These images confirm that the coagulum which clogs the slit consists of PMMA as well as PU particles.
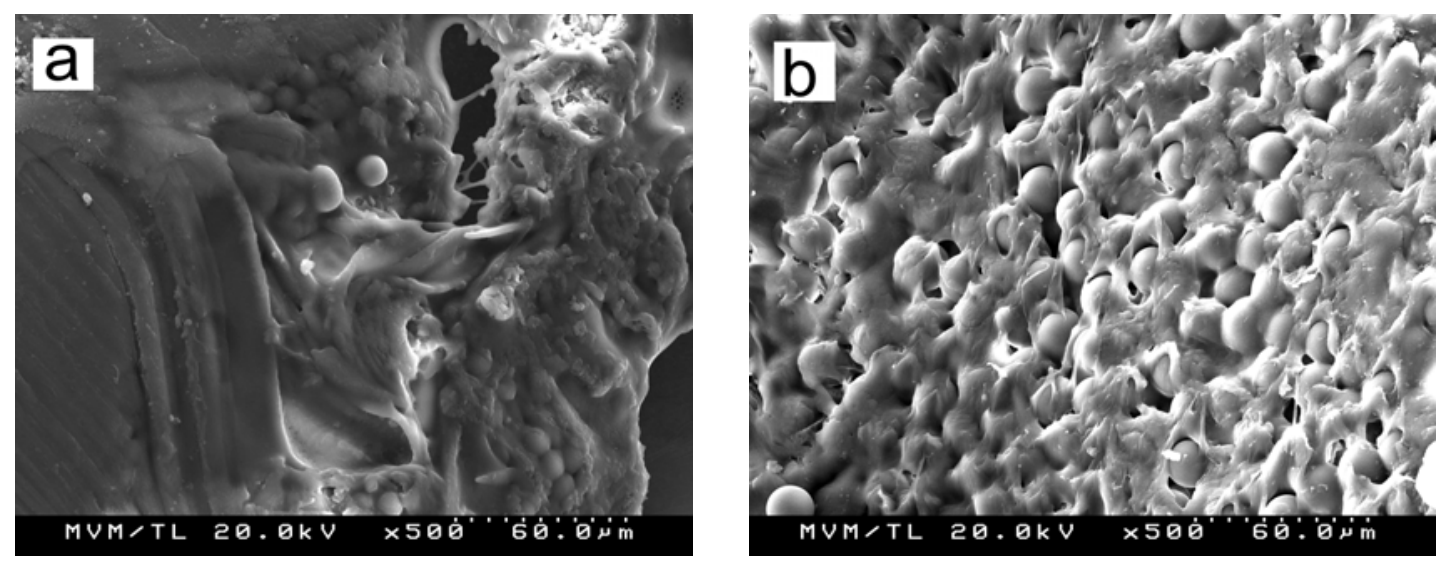

Figure 27. Electron micrographs of the coagulum after the ring-slit test of a PU-dispersion with (a) 100 ppm PMMA and (b) 1000 ppm PMMA particles (10 $\mu \mathrm{m})$.

In Figure 28a the effect of PMMA particle size is displayed for three different particle sizes $(1 \mu \mathrm{m}, 10 \mu \mathrm{m}$ and $14.5 \mu \mathrm{m}$ diameter). In these experiments, the volume fraction of PMMA particles is fixed at $100 \mathrm{ppm}$ and the hydrodynamic conditions are the same as in the experiments described above. It is evident that the size of the PMMA particles has no significant effect on dispersion stability. The strong pressure increase observed with the $1 \mu \mathrm{m}$ particles is especially striking since these particles are twenty times smaller 
than the ring-slit gap. Figure $28 \mathrm{~b}$ demonstrates the dependence of the PU suspension stability on the amount of $1 \mu \mathrm{m}$ PMMA particles. It can be seen that the rate of slit clogging decreases with decreasing PMMA particle fraction; a critical amount of PMMA particles is required to induce the clogging phenomenon.
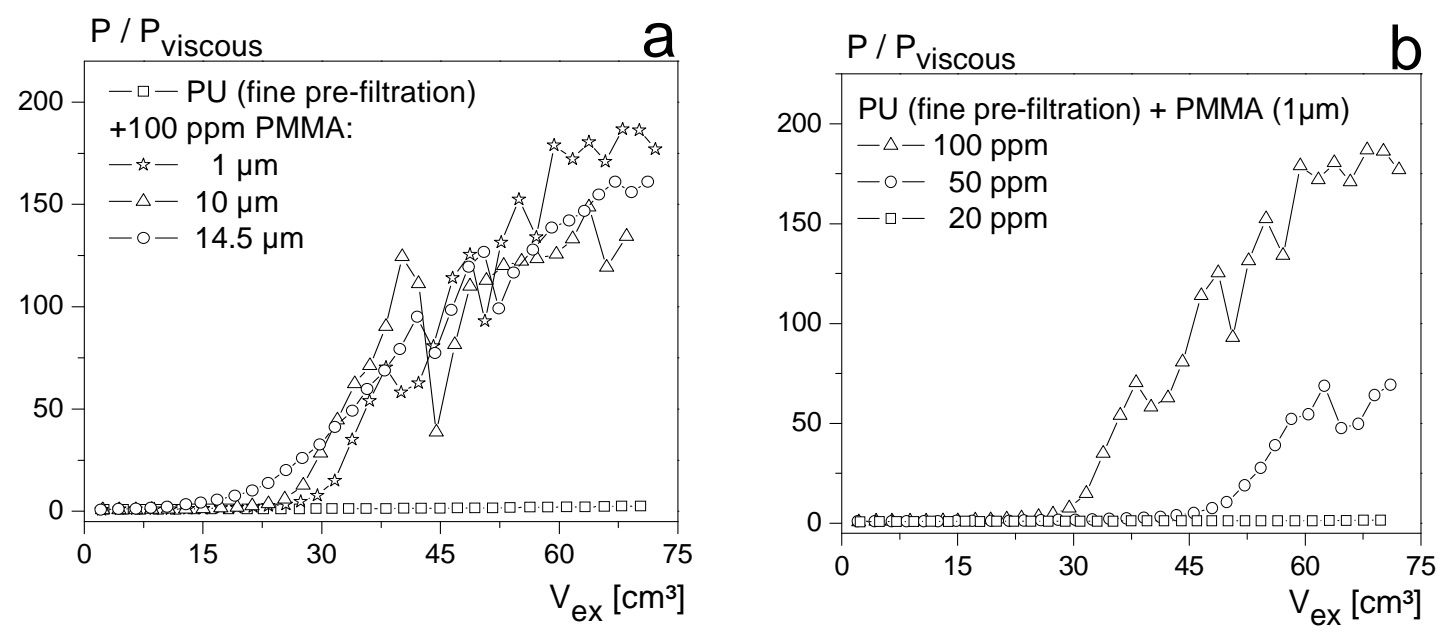

Figure 28. Effect of PMMA particles size (100 ppm) (a) and PMMA particle (1 $1 \mu \mathrm{m})$ volume fraction (b) on the flow stability of the PU dispersion (fine filter, $\phi=0.4, H=21 \mu \mathrm{m}, \beta=45^{\circ}$ and flow rate $=314 \mathrm{~mm}^{3} / \mathrm{s}$ )

Figure 29a shows the dependence of polychloroprene flow stability on the PMMA particle size. For these experiments, 1000 ppm PMMA particles were dispersed in the suspension and the samples were extruded through a $21 \mu \mathrm{m}$ slit at a flow rate of $314 \mathrm{~mm}^{3} / \mathrm{s}$. A clear effect of PMMA particle size on polychloroprene stability can be seen in this case and the pressure increase is more pronounced as the ratio of particle size to gap width decreases. Despite the high PMMA particle concentration the magnitude of the normalized pressure in these experiments is much smaller than that for the PU suspension shown in Figure 28a. The implication of this result is that the polychloroprene particles are better stabilized than the PU particles and the aggregation efficiency between PMMA and polychloroprene particles is greatly reduced, which is actually a further argument for the hetero-coagulation mechanism in the ring-slit device. The effect of the PMMA particle volume fraction on the flow-induced aggregation of the polychloroprene dispersion is demonstrated for the $14.5 \mu \mathrm{m}$ PMMA particles in Figure 29 b. As expected, normalized pressure decreases with decreasing PMMA particle volume fraction. 

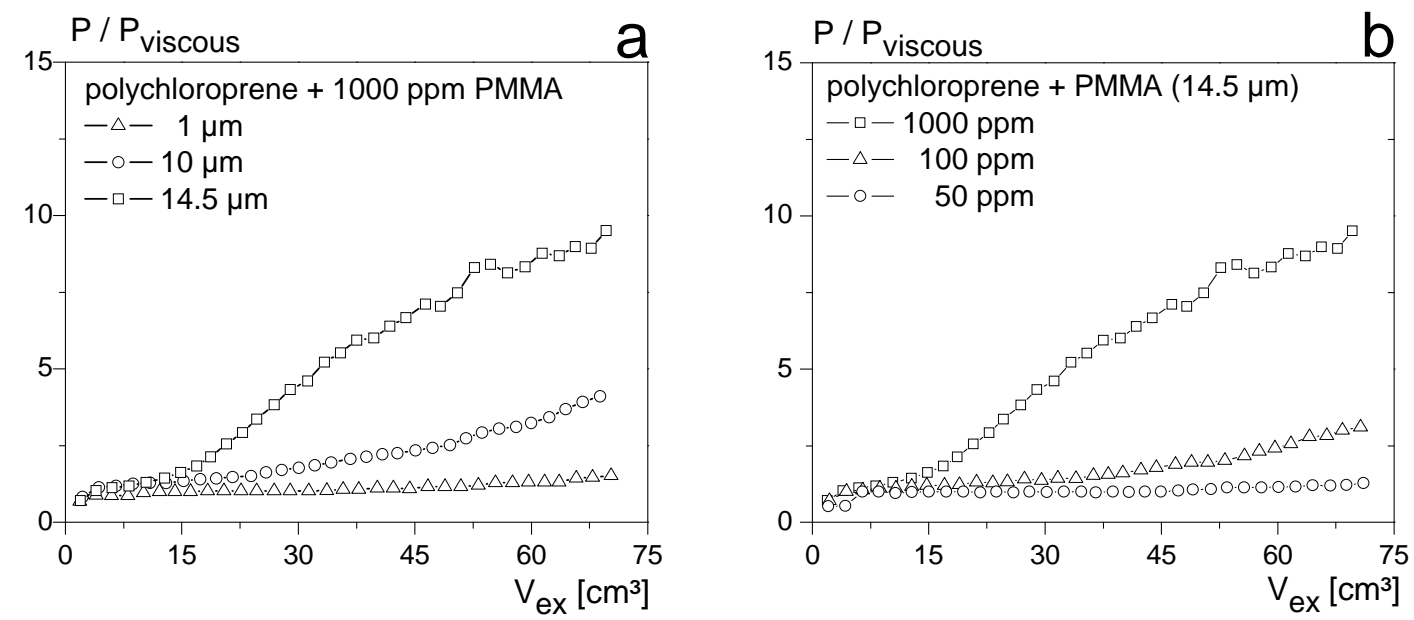

Figure 29. Effect of PMMA particles size (1000 ppm) (a) and PMMA (14.5 $\mu \mathrm{m})$ particle volume fraction (b) on the flow induced aggregation in the polychloroprene suspension (fine filter, $\phi=$ $0.5, H=21 \mu \mathrm{m}, \beta=45^{\circ}$ and flow rate $=314 \mathrm{~mm}^{3} / \mathrm{s}$ )

\subsubsection{Effect of particle concentration}

Figure 30 shows the effect of PU particle concentration on particle retention for a $21 \mu \mathrm{m}$ slit and constant volumetric flow rate $\left(314 \mathrm{~mm}^{3} / \mathrm{s}\right)$. In these experiments the polyurethane dispersion was coarse pre-filtered and diluted to different concentrations using $\mathrm{KCl}$ solution $(0.01 \mathrm{M})$. When normalized pressure drop across the slit is plotted as a function of the volume of particles extruded $\left(\mathrm{V}_{\mathrm{ex}}{ }^{*} \phi\right)$, the data obtained for different particle volume fractions superimpose very well, which means that slit clogging is controlled by the total number of particles that have passed the slit, but the efficiency of particle retention does not depend on the particle concentration. Similar results are obtained for both lower and higher flow rates (see appendix 6.1.6). These results can be explained by the hetero-coagulation between micron-sized particles and primary particles. Since the number of primary particles is infinite in comparison to the micron-sized particles, hetero-coagulation should depend linearly on the concentration of micrometer objects, assuming that the aggregation efficiency is high enough. Furthermore, the linear dependence on particle volume fraction indicates that hetero-coagulation predominates over the coagulation of the aggregates themselves, for which the aggregation rate should be proportional to the second power of concentration. 


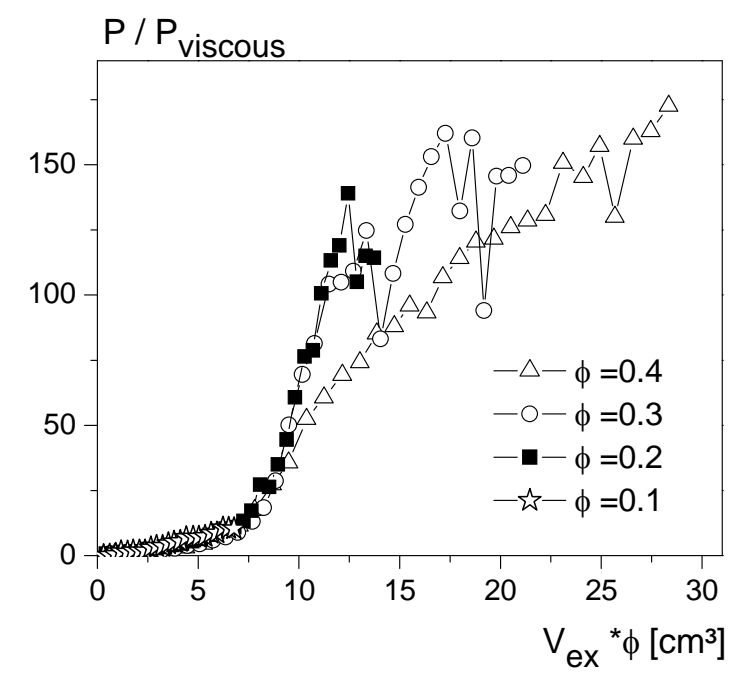

Figure 30. Effect of volume fraction on the clogging phenomenon for the PU dispersion (coarse filter, $H=21 \mu \mathrm{m}, \beta=45^{\circ}$ and flow rate $=314 \mathrm{~mm}^{3} / \mathrm{s}$ )

Finally, clogging by particle-wall interactions or size exclusion could also explain the linear concentration dependence of the pressure increase but all experimental results presented previously confirm that these phenomena are not relevant for the experiments discussed here.

\subsection{Impact of colloidal properties on flow induced aggregation}

The emphasis of this section is on the influence of the colloidal properties of the nanoparticles, which play a crucial role in dispersion stability. The repulsive potential barrier should be large enough that it can not be overwhelmed by the hydrodynamic forces to prevent flowing particles from agglomeration. In order to investigate the impact of colloidal particle interactions, we tested the effect of surfactant adsorption and ionic strength on the flow-induced aggregation.

\subsubsection{Effect of surfactant on flow induced aggregation}

The adsorption of surfactant molecules on colloidal particles provides steric and electrostatic repulsion depending on the surfactant type. The influence of the surfactant on the flow-induced coagulation of the PU dispersion (coarse pre-filtration) was studied for an anionic SDS surfactant and for a non-ionic surfactant based on polyglycol ether. Figure 31a shows the effect of the SDS surfactant for three different concentrations at a flow rate of $314 \mathrm{~mm}^{3} / \mathrm{s}$ and a slit height of $21 \mu \mathrm{m}$. This data demonstrate that flow stability increases upon addition of SDS in the range of $11-27 \mathrm{mmol} / 1$ (with reffernce to the volume of dispersion medium). As the SDS surface density progressively increases, both the repulsive steric potential energy and the electrical double layer repulsion are in- 
creased, leading to a decrease in particle aggregation efficiency. When the surface is fully covered, the hydrodynamic forces can not overcome the repulsive potential and the dispersion is stable [95][96]. Indeed, we have estimated that at $27 \mathrm{mmol} / \mathrm{l}$ surfactant concentration all the primary PU particles are fully covered. A similar affect of the SDS surfactant on flow-induced aggregation was obtained for the PS dispersion (coarse prefiltraton) as well as for the PU suspension in the presence of PMMA particles (see Appendix 6.1.5). Figure $31 \mathrm{~b}$ shows that the non-ionic polymeric surfactant also improves the flow stability of the coarse pre-filtered PU suspension. The PU particles are entirely covered by the non-ionic surfactant at lower surfactant concentration due to the much lower critical micelle concentration (about $0.3 \mathrm{mmol} / \mathrm{l}$ ) in comparison to that of SDS surfactant (about $5 \mathrm{mmol} / \mathrm{l}$, estimated considering the dispersion ionic strength 8 mmol/l) . Recently, Wu et al. [97][98] have extensively studied the effect of surfactant on flow induced aggregation, and have found that aggregation is impossible when sufficient surfactant is adsorbed on the particle surface. They pointed out that the adsorption of a given surfactant system on the particle surface generates very strong short-range hydration force that is able to prevent shear-induced aggregation even when the work done by the shear stress is higher than the energy barrier predicted by the DLVO-theory.
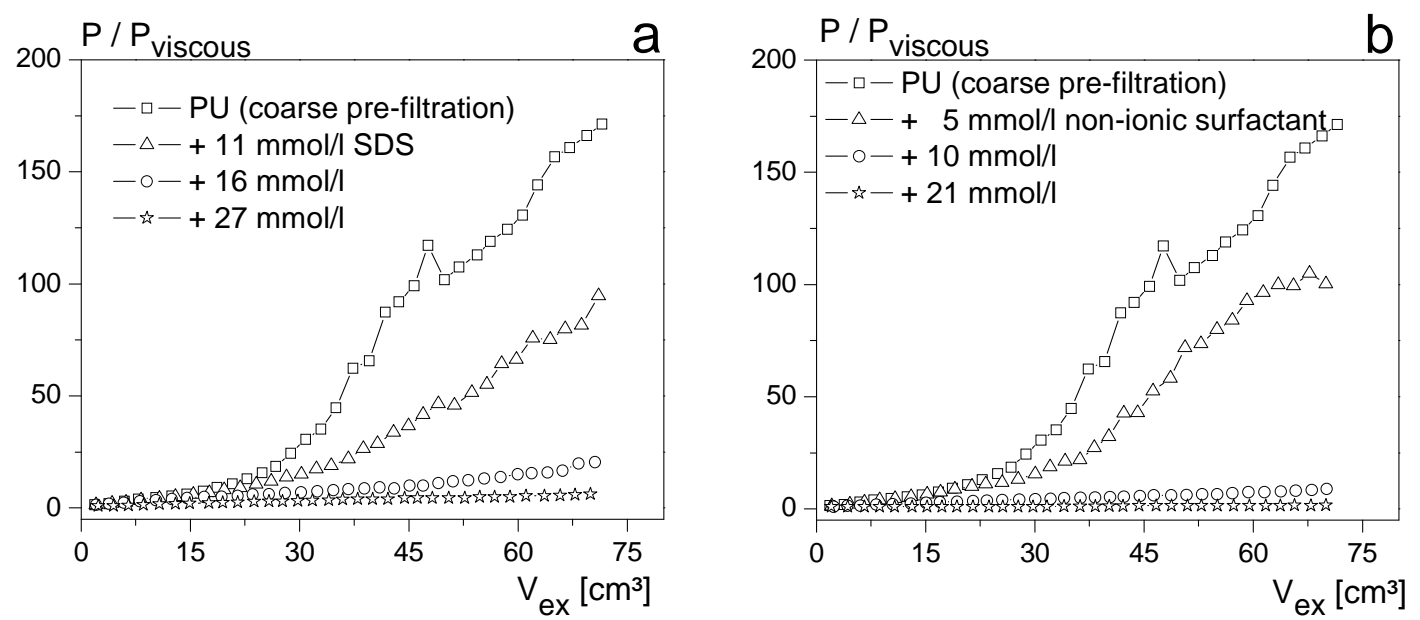

Figure 31. Effect of anionic SDS surfactant (a) and non-ionic polymeric surfactant (b) on the flow stability of PU-dispersion (coarse filter, $\phi=0.4, H=21 \mu \mathrm{m}, \beta=45^{\circ}$ and flow rate $=314$ $\mathrm{mm}^{3} / \mathrm{s}$ )

The thickness of a surfactant layer, relative to the particle size, is an essential factor for an effective steric stabilization. Since the van der Waals attraction force is proportional to particle size, larger particles will need thicker stabilizing layers to prevent the approach of the particles within the range of the attractive van der Waals forces [14]. The attractive force between two $1 \mu \mathrm{m}$ particles is higher by a factor of 10 than that experienced between $100 \mathrm{~nm}$ particles at a given particle surface separation. Typically, a surfactant layer thickness of a tenth of the particle radius is needed to prevent irreversible 
particle aggregation [14][99][100]. Accordingly, effective steric stabilization of the large micrometer aggregates by the relatively small surfactant molecules is very unlikely. Therefore, we can argue that the improved stability of the primary nanoparticles is due to the added surfactant reducing the flow-induced aggregation. This further supports the idea of hetero-coagulation.

\subsubsection{Effect of ionic strength}

Another important factor which influences dispersion stability, is the ionic strength. Dialysis against salt solutions of defined ionic strength (see section 3.2.1.2.1) allows us to tune the range of electrostatic repulsion [14]. Figure 32 illustrates the effect of ionic strength on the flow stability of the PU dispersion (coarse pre-filtration). The displayed data were obtained at volumetric flow rate of $314 \mathrm{~mm}^{3} / \mathrm{s}$ and a slit height of $21 \mu \mathrm{m}$, but similar results were found in the whole flow rate range investigated here. As expected, the pressure increase is more pronounced, when the range of electrostatic interaction is reduced.

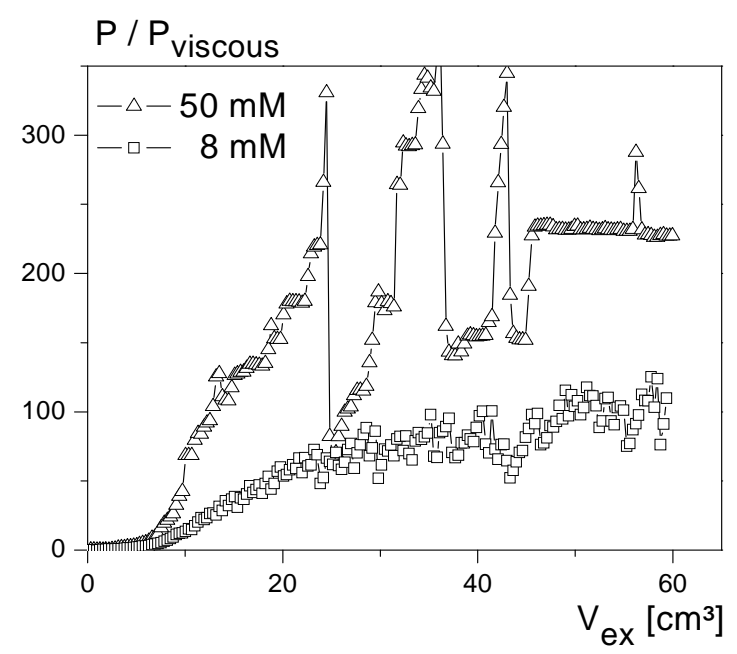

Figure 32. Effect of ionic strength on the flow stability of PU-dispersion (coarse filter, $\phi=0.4$, $H=21 \mu \mathrm{m}, \beta=45^{\circ}$ and flow rate $=314 \mathrm{~mm}^{3} / \mathrm{s}$ )

\subsubsection{Effect of $\mathrm{pH}$}

We have shown in section 3.2.1.2 that the zeta-potential of the polychloroprene particles is $\mathrm{pH}$ dependent. With decreasing $\mathrm{pH}$ the degree of ionization of the carboxylic surface groups decreases leading to a decrease in the surface charge density. The $\mathrm{pH}$ of the polychloroprene suspension was adjusted by adding citric acid ( $0.2 \mathrm{M})$ dropwise to the suspension. Vigorous stirring was applied to mix the acid and avoid local aggregation. However, the results from the SPOS analysis revealed that a large amount of micron sized aggregates have been formed during the $\mathrm{pH}$ adjustment of the polychloroprene suspension. Figure 33 shows the volume-weighted differential distribution of micron- 
sized objects for the initial suspension at a $\mathrm{pH}$ of 12 and after the addition of acid to a $\mathrm{pH}$ of 8 (note that the samples have been fine pre-filtered). It is evident that the sample at $\mathrm{pH}$ of 8 contains a small amount of particles larger than in the initial suspension as well as larger relative volume fraction of aggregates with sizes between 1-2 $\mu \mathrm{m}$ in diameter. Indeed, evaluation of the volume fraction of measured aggregates showed an enormous increase of the aggregate concentration from $37 \mathrm{ppm}$ in the sample at $\mathrm{pH} 12$ (Table 5) to $427 \mathrm{ppm}$ (referred to the volume of dispersion medium) after the $\mathrm{pH}$ adjustment to a $\mathrm{pH}$ of 8 .

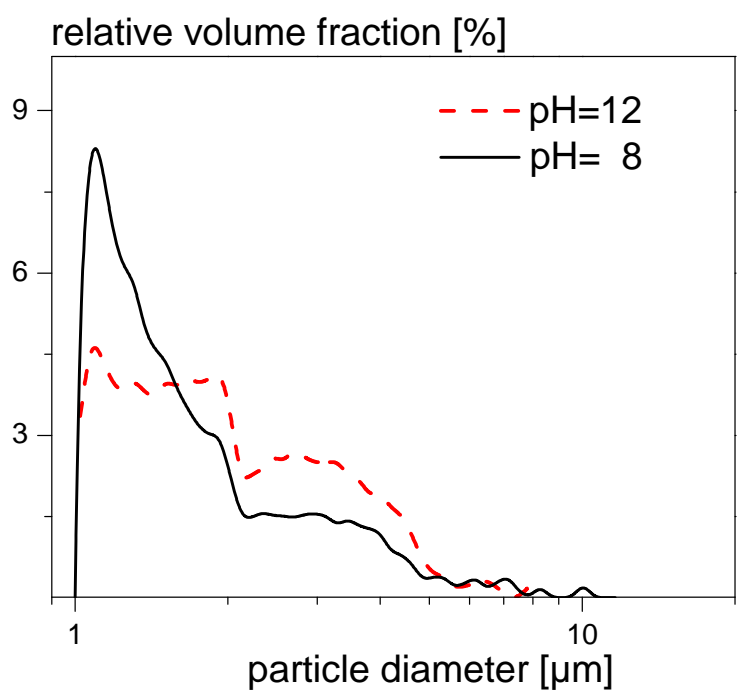

Figure 33. Volume-weighted differential distribution of micron-sized particles in the polychloroprene suspension (fine pre-filtration) at a pH of 12 and after the addition of acid to a $\mathrm{pH}$ of 8 from the measurements using AccuSizer $780 \mathrm{~A}$

Figure 34 shows the effect of $\mathrm{pH}$ on the rate of polychloroprene particle aggregation at fixed ionic strength. Note that the $\mathrm{pH}$ value was adjusted just before the ring-slit test. These experiments were performed extruding the samples through a $21 \mu \mathrm{m}$ slit at 314 $\mathrm{mm}^{3} / \mathrm{s}$ volumetric flow rate. It can be seen that the normalized pressure drop increases with decreasing the $\mathrm{pH}$ value. Obviously, the flow stability of the polycloroprene dispersion decreases as the $\mathrm{pH}$ becomes less alkaline and can be explained by decreasing surface charge density leading to a reduction of the repulsive energy barrier that prevents particles from aggregation. However, it should be noted that these results are not conclusive, since the observed effect may also be due to hetero-coagulation as a result of the increased aggregate concentration after the $\mathrm{pH}$ adjustment. 


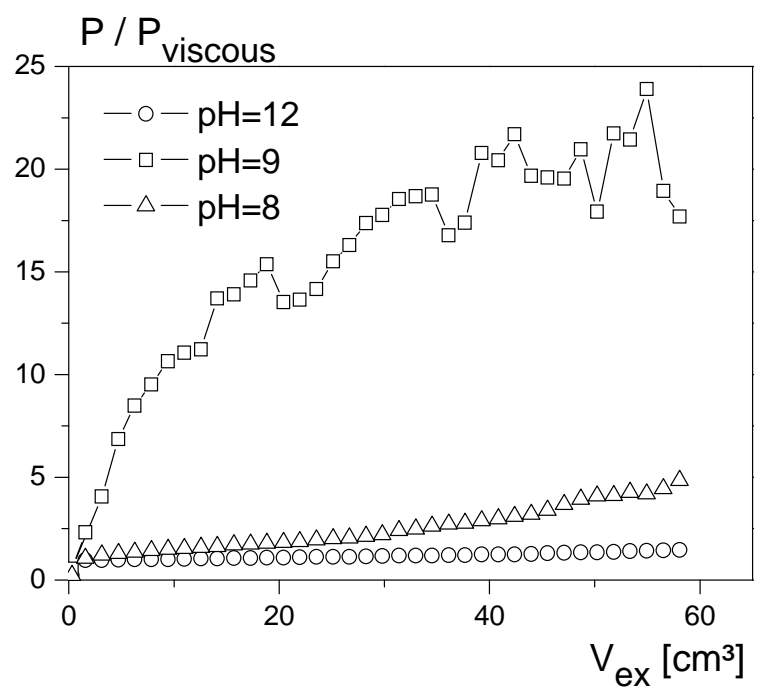

Figure 34. Effect of $\mathrm{pH}$ on the flow stability of the polychloroprene dispersion (fine filter, $\phi=$ $0.5, H=21 \mu \mathrm{m}, \beta=45^{\circ}$ and flow rate $=314 \mathrm{~mm}^{3} / \mathrm{s}$ )

\subsection{Effect of slit height}

Figure 35 shows the effect of slit height on the particle retention at the slit entrance for the investigated suspensions. In these experiments the suspensions were extruded at a constant flow rate $\left(63 \mathrm{~mm}^{3} / \mathrm{s}\right)$ using a slit die with a $45^{\circ}$ entrance angle. The results presented in Figure 35 show a significant dependence of the normalized pressure drop on the slit height. In the case of the PU and PS suspensions (coarse pre-filtration), a great pressure increase was observed when the samples were extruded through the $21 \mu \mathrm{m}$ slit; no aggregate formation was detected at $26 \mu \mathrm{m}$ slit height (Figure 35a and Figure 35b). Aggregate formation in the polychloroprene suspension (fine pre-filtration) was detected at a $11 \mu \mathrm{m}$ slit. These results can be explained by considering the size distribution of pre-existing micron-sized objects (Figure 18): The PU and PS suspensions contain larger particles with median diameter $\left(\mathrm{d}_{50}\right)$ of $9 \mu \mathrm{m}$ and $6 \mu \mathrm{m}$ respectively, whereas the micron-sized particles in the polychloroprene dispersion are significantly smaller $\left(\mathrm{d}_{50}=1.5 \mu \mathrm{m}\right)$. As the probability of forming aggregates larger than the slit height decreases with increasing the aspect ratio (defined as the ratio of the slit height to the diameter of micron-sized particles), the retention of aggregates at the slit entrance becomes insignificant at sufficiently high aspect ratios. The results in Figure 35 demonstrate, for the investigated suspensions, that the extent of slit clogging changes dramatically in a very narrow range of the aspect ratio. This further confirms the significance of hetero-coagulation of micron-sized and nano-particles. The primary particles are about two orders of magnitude smaller in size than the micron-sized impurities and contribute only slightly to the increase of the formed aggregates. Note that these results are in agreement with the effect of pre-filtration on the flow induced aggregation. Although, the fine pre-filtration of the PU and PS samples causes only about $5 \mu \mathrm{m}$ reduction of the 
maximum particle size in comparison to the coarse filter (Figure 18), it has a dramatic effect on the clogging phenomenon (Figure 25). It should be mentioned again that the measured particle diameter is overestimated due to the irregular, predominantly elongated shape of micron-sized objects (see section 3.2.1.1). This makes the analysis of the effect of pre-filtration and slit height on the clogging phenomenon difficult, since the longest aggregate dimension is aligned with the direction of the flow at the slit entrance (the Peclet number at the slit entrance is much larger than unity, even at the lowest flow rate) and information about the cross-sectional diameter of the elongated aggregates is not available.
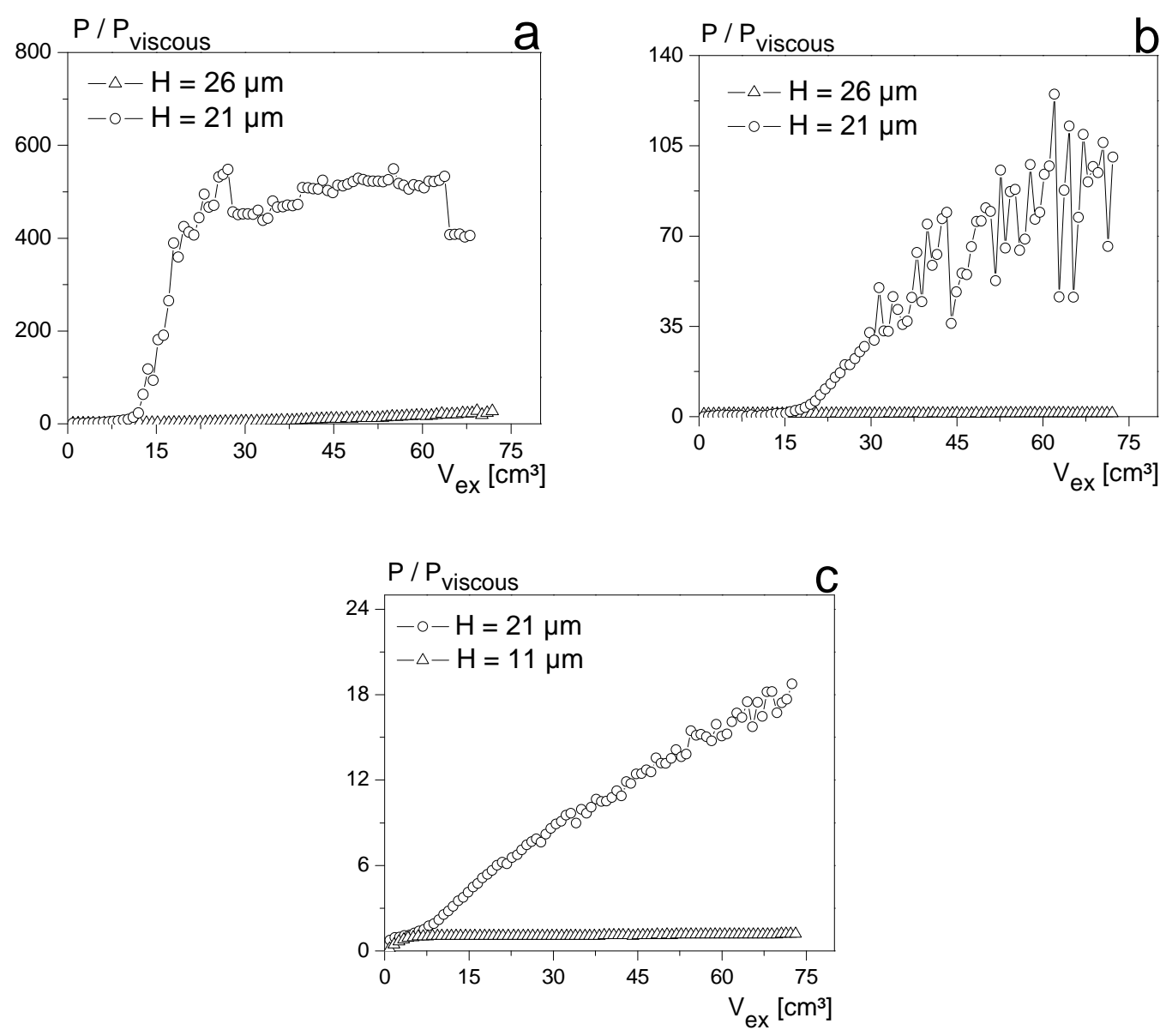

Figure 35. Effect of the slit height on the flow-induced aggregation of the coarse pre-filtered $P U$ dispersion at $\phi=0.4, \beta=45^{\circ}$ and flow rate $=63 \mathrm{~mm}^{3} / \mathrm{s}$ (a), coarse pre-filtered PS dispersion at $\phi=0.47, \beta=45^{\circ}$ and flow rate $=63 \mathrm{~mm}^{3} / \mathrm{s}(\mathrm{b})$, and coarse pre-filtered polychloroprene dispersion at $\phi=0.5, \beta=45^{\circ}$ and flow rate $=63 \mathrm{~mm}^{3} / \mathrm{s}(\mathrm{c})$ 


\subsection{Flow kinematics parameter and their influence on flow- induced aggregation}

\subsubsection{Interpretation of the effect of flow rate on flow induced aggregation}

In the previous sections we have experimentally demonstrated that the slit clogging is due to hetero-coagulation in the converging flow field at the slit entrance and the slit clogs faster for lower flow rate (Figure 23). In this section, we will consider the agglomeration and the agglomerate breakage mechanism under high shear conditions in order to gain a better understanding of the effect of flow rate.

Flow-induced agglomeration of strongly repulsive particles requires that the hydrodynamic force acting on particles is large enough to overcome the repulsive interaction energy barrier $\left(\Psi_{\max }\right)$. In a recent work, Zaccone et al. pointed out that a critical Peclet number exists [101]:

$$
P e_{c}=\frac{\Psi_{\max }}{2 i k_{B} T}
$$

where $i$ is a coefficient that accounts for the flow kinematics. The basic idea is that when the Peclet number of the sheared suspension is greater than $P e_{c}$, the work done by the shear stress is greater than the energy barrier provided by the colloidal forces and the aggregation process is activated, otherwise the aggregation process is reaction limited and very slow.

To find $\mathrm{Pe}_{\mathrm{c}}$, we estimated $\Psi_{\max }$ from the total colloidal interaction energy according to the classical DLVO theory accounting for the short range Born repulsion:

$$
\Psi=\Psi_{e l}+\Psi_{v d W}+\Psi_{\text {Born }}
$$

The electrostatic repulsive potential $\left(\Psi_{e l}\right)$ was computed using the modified HoggHealy-Fuerstenau approximation derived by Sader et al. (11). For these calculations, the surface potential $\psi_{0}$ is assumed to be equal to the measured zeta-potential, and the Debye length, $\mathrm{\kappa}^{-1}$, was set to the values estimated rheologically as reported in section 3.2.1.4. We used equation (7) to estimate the van der Waals attractive potential, which considers the interaction of spherical particles of different radius. The value of the Hamaker constant $\left(A_{H}\right)$ is assumed to be equal to the one for polystyrene $\left(1.3 \times 10^{-20} \mathrm{~J}\right)$ for all of the investigated colloidal systems. To compute $\Psi_{\text {Born }}$, we use the expression made by Feke et al. (equation (13)), where the parameter $\mathrm{N}_{12}$, expressing the strength of the van der Waals attraction relative to the Born repulsion, is set to equal $1 \times 10^{-21}$ [103]. Figure 36 shows the total interaction energy for the investigated suspensions as a func- 
tion of the separation distance, $\mathrm{h}$, computed according to equation (82). Using the estimated $\Psi_{\max }$ values (Table 9) and setting the coefficient $i$ equal to 0.19 , by assuming an axisymmetrical extensional flow [102], we obtain the corresponding critical Peclet number values, which are $\mathrm{Pe}_{\mathrm{c}}=758,689$ and 592 for the PU, PS and polychloroprene suspension, respectively.

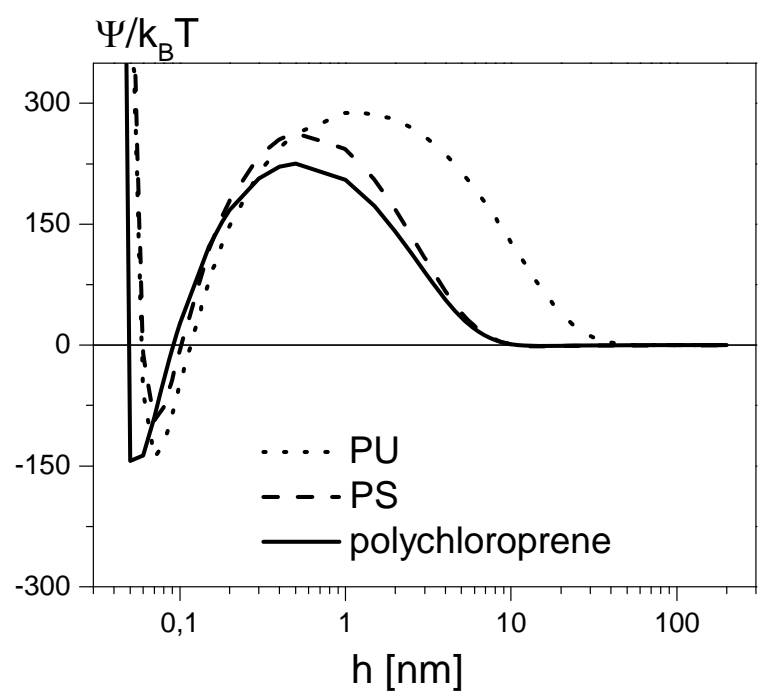

Figure 36. Interparticle interaction potential, $\Psi / k_{B} T$, as a function of the interparticle separation distance, $h$, for the PU, PS and polychloroprene particles, computed using the parameters reported in Table 9.

In order to compare the Peclet number for our experimental conditions with $P e_{c}$, we have calculated the Peclet number for homo-coagulation:

$$
P e_{p, p}=\frac{6 \pi \eta a_{p}^{3} \dot{\gamma}}{k_{B} T}
$$

where $a_{p}$ is the radius of the primary nano-particles, and for hetero-coagulation:

$$
P e_{p, i}=\frac{3 \pi \eta \dot{\gamma}\left(a_{p}+a_{i}\right) a_{p} a_{i}}{k_{B} T}
$$

where $a_{i}$ is the radius of the micron-sized impurities. For $a_{i}=1 \mu \mathrm{m}$ and $a_{p}=70 \mathrm{~nm}$ the obtained $\mathrm{Pe}_{\mathrm{p}, \mathrm{p}}$ and $\mathrm{Pe}_{\mathrm{p}, \mathrm{i}}$ values are 105 and 610294, respectively, at the slit wall shear rate, $\dot{\gamma}=6700 \mathrm{~s}^{-1}$ which corresponds to the lowest flow rate $\left(23 \mathrm{~mm}^{3} / \mathrm{s}\right)$. Thus, the critical Peclet number is exceeded for all investigated systems if micron-sized impurities are present in the suspension, further supporting the argument for hetero-coagulation as is evident by the experimental results (Figure 26 - Figure 29). Furthermore, these considerations suggest the formation of aggregates even for the fine pre-filtered samples. Indeed, SPOS analysis of the fine pre-filtered PU dispersion after extrusion at the lowest flow rate revealed an increase in the agglomerate volume fraction, even though the slit 
was not clogged. On the other hand, $\mathrm{Pe}_{\mathrm{p}, \mathrm{i}}$ also increases when increasing the shear rate, suggesting that the particle aggregation should become more likely at high flow rates, which is in contradiction to the results shown in Figure 23. The effect of flow rate on the slit clogging is understood by considering the effect of interparticle contact time on the aggregation probability and the process of aggregate breakage leading to a reduction of the maximum aggregate size.

Chesters [49] argued that the aggregation probability depends not only on the collision force but also the collision duration. As discussed in section 2.3.2.2, equation (66) provides an estimation of the aggregation probability based on the time spent by two particles in immediate vicinity and the time required for drainage of the fluid in the small gap between approaching particles. In order to calculate the aggregation probability for our colloidal systems, we replaced the term $a^{3}$ in equation (66) by $2\left(a_{p}+a_{i}\right) a_{p} a_{i}$, to account for the aggregation between unequal particles. Increasing flow rate causes the aggregation probability, $P$, for $\mathrm{a}_{\mathrm{p}}=70 \mathrm{~nm}$ and $\mathrm{a}_{\mathrm{i}}=1 \mu \mathrm{m}$, to decrease from an estimated $39 \%$ at $23 \mathrm{~mm}^{3} / \mathrm{s}$, to $23 \%$ at $1570 \mathrm{~mm}^{3} / \mathrm{s}$.

Table 9. Colloidal interaction parameters for the investigated suspensions

\begin{tabular}{lcccccc}
\hline Dispersion & $\Psi_{\mathbf{0}}$ & $\mathbf{1} / \mathbf{\kappa}$ & $\mathbf{A}_{\mathbf{H}}$ & $\mathbf{N}_{\mathbf{1 2}}$ & $\Psi_{\mathbf{m a x}} / \boldsymbol{k}_{\mathbf{B}} \boldsymbol{T}$ & $\Psi_{\mathbf{m i n}} / \boldsymbol{k}_{\mathbf{B}} \boldsymbol{T}$ \\
& {$[\mathbf{V}]$} & {$[\mathbf{m}]$} & {$[\mathbf{J}]$} & & & \\
\hline Polyurethane & 0.056 & $1 \times 10^{-8}$ & $1.3 \times 10^{-20}$ & $1 \times 10^{-21}$ & 300 & -127 \\
Polystyrene & 0.059 & $1 \times 10^{-9}$ & $1.3 \times 10^{-20}$ & $1 \times 10^{-21}$ & 263 & -94 \\
Polychloroprene & 0.062 & $1 \times 10^{-9}$ & $1.3 \times 10^{-20}$ & $1 \times 10^{-21}$ & 225 & -143 \\
\hline
\end{tabular}

Another important aspect when considering suspensions exposed to shear stress is the breakage of aggregates. In order to estimate the resistance of aggregates against breakage, we estimated the Breakage number, Br, as suggested by Xie et al [102]. They expressed the Breakage number as the ratio between work done by the shear stress and interparticle bonding energy. Considering a hetero-coagulation process, the Breakage number can be written in the following form:

$$
B r=\left(\frac{\eta \dot{\gamma}\left(\mathrm{a}_{\mathrm{p}}+\mathrm{a}_{\mathrm{i}}\right) \mathrm{a}_{\mathrm{p}} \mathrm{a}_{\mathrm{i}}}{\Psi_{b}}\right)
$$

where $\Psi_{\mathrm{b}}$ is the interparticle bonding energy associated with the difference between the interaction energy barrier, $\Psi_{\max }$, and the primary minimum, $\Psi_{\min }$ :

$$
\Psi_{b}=\Psi_{\text {max }}-\Psi_{\text {min }}
$$


The values of $\Psi_{\max }$ and $\Psi_{\min }$, calculated according to the procedure described above, and the values of all parameters used for the calculation are summarized in Table 9. The $\mathrm{Br}$ values calculated for $\mathrm{a}_{\mathrm{p}}=70 \mathrm{~nm}$ and $\mathrm{a}_{\mathrm{i}}=1 \mu \mathrm{m}$ using equation (85) are 2 and 145 at 23 $\mathrm{mm}^{3} / \mathrm{s}$ and $1570 \mathrm{~mm}^{3} / \mathrm{s}$ respectively. The Breakage number is always larger than unity and increases as the flow rate increases for primary particle and impurity sizes relevant for the investigated colloidal systems. Hence, for the experimental conditions in the ring-slit test, the applied shear energy is sufficient to overcome the interparticle bonding energy and the average aggregate size decreases as the shear energy or Breakage number increases. However, aggregate breakage is a complex phenomenon, the treatment of which should include the concept of decreased aggregation efficiency as well as largescale defragmentation and surface erosion. This is especially relevant for the extensional flow through the contraction region in our ring-slit die, which continuously accelerates and reaches a maximum elongation rate at the slit entrance. This means that the aggregates formed under low-strain conditions may breakup when exposed to the larger elongation rate in the proximity of the slit. Moreover, detailed considerations of multiparticle interactions would be necessary for a full quantitative description of flowinduced aggregation, particularly for the case of highly concentrated suspensions investigated here.

Let us return to the results showing the effect of flow rate on the slit clogging phenomenon (Figure 23). Based on the considerations discussed above we expect that the increase of the flow rate causes a decrease of the maximum aggregate size, which is controlled by the balance between flow-induced aggregation and breakage in the converging flow field at the slit entrance. Thus, a certain fraction of aggregates smaller than the slit height can pass the gap This is evidenced in Table 7 for the PU suspension.

\subsubsection{Effect of entrance angle on flow induced aggregation}

We studied the effect of the slit entrance angle on flow induced aggregation to further investigate the effect of flow kinematics. Figure 37 shows the influence of the entrance angle for the three colloidal systems at a $63 \mathrm{~mm}^{3} / \mathrm{s}$ volumetric flow rate. The results for the PU and PS suspensions are obtained at $26 \mu \mathrm{m}$ slit height (Figure 37a and b), where samples are coarse pre-filtered before the ring-slit experiments. In the case of the polychloroprene suspension (fine pre-filtration), the slit height was fixed to $11 \mu \mathrm{m}$ (Figure 37 c). The results presented in Figure 37 show a significant dependence of the normalized pressure drop on the slit entrance angle. For all colloidal systems, the pressure increase is most pronounced in the case of $30^{\circ}$ slit entrance angle. In the cases of PU and PS, the results for entrance angles of $45^{\circ}$ and $90^{\circ}$ almost superimpose, while in the case of the polychloroprene sample a continuous increase of the flow stability was detected with increasing the entrance angle. 

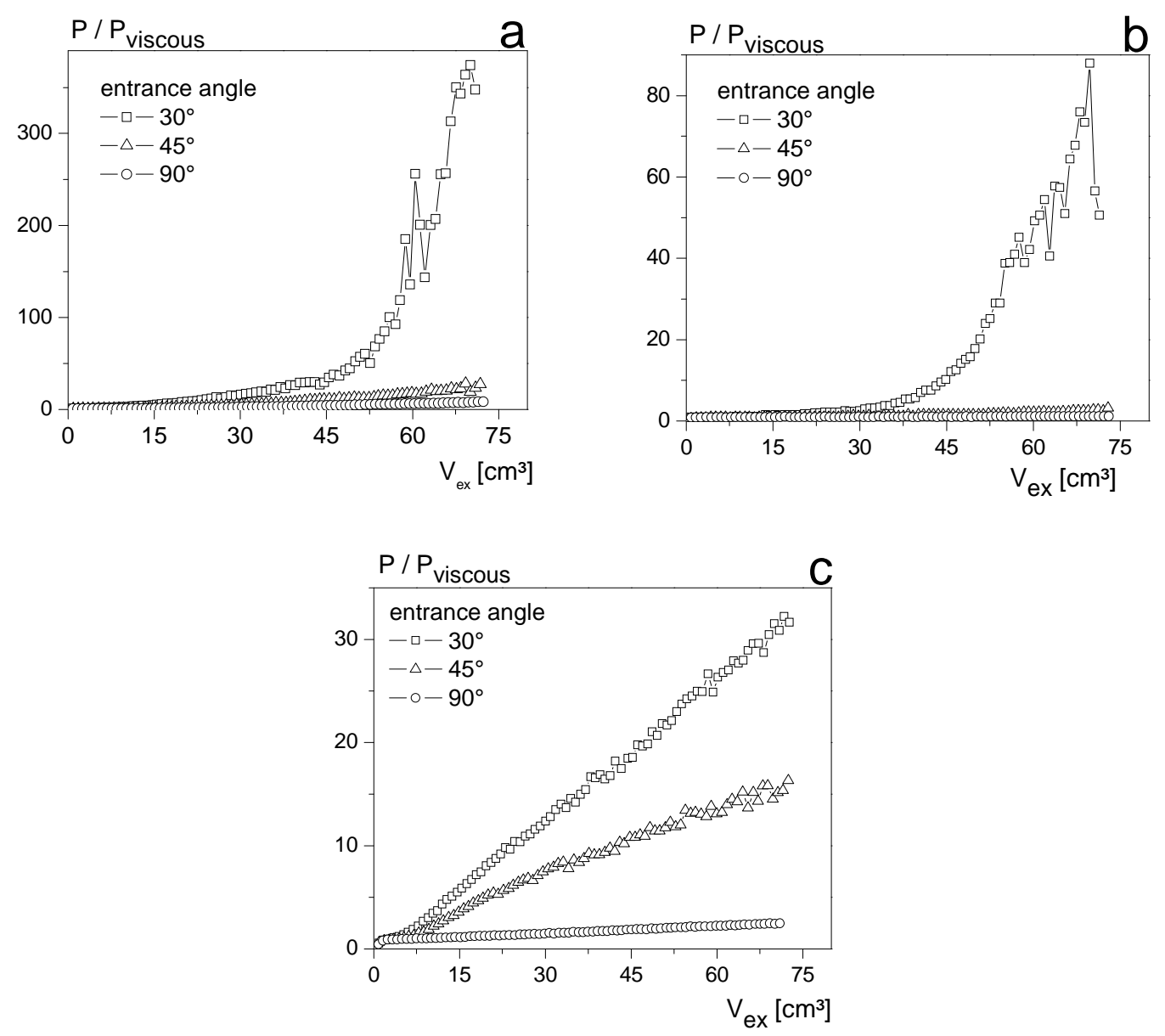

Figure 37. Effect of the slit entrance angle on the flow-induced aggregation of the coarse prefiltered PU dispersion at $\phi=0.4, H=26 \mu \mathrm{m}$ and flow rate $=63 \mathrm{~mm} / \mathrm{s}(\mathrm{a})$, coarse pre-filtered $P S$ dispersion at $\phi=0.47, H=26 \mu \mathrm{m}$ and flow rate $=63 \mathrm{~mm}^{3} / \mathrm{s}(\mathrm{b})$ and coarse pre-filtered polychloroprene dispersion at $\phi=0.5, \mathrm{H}=11 \mu \mathrm{m}$ and flow rate $=63 \mathrm{~mm}^{3} / \mathrm{s}$ (c)

We need to characterise the flow field in the ring-slit device in order to understand the effect of slit entrance angle on the flow-induced aggregation. For this purpose, we used the commercial CFD software Ansys Fluent 12. The 2D axisymmetric simulations were focused on the contracting region at the entrance of the slit with the following dimensions: $\mathrm{L}=1.2 \mathrm{~mm}, \mathrm{~L}=0.8 \mathrm{~mm}, \mathrm{H}=26 \mu \mathrm{m}$ and $\beta=30^{\circ}, 45^{\circ}$ and $90^{\circ}$ (see Figure $8 \mathrm{~b}$ ). The viscosity of the investigated shear-thinning suspensions is well described by the Carreau model, $\eta=\eta_{\infty}+\left(\eta_{0}+\eta_{\infty}\right)\left(1+(\lambda \dot{\gamma})^{2}\right)^{(\mathrm{n}-1) / 2}$. For calculations shown in Figure 38 we have used the following model parameters determined for the PU dispersion with $\phi=0.4: \eta_{0}$ $=50$ Pas, $\eta_{\infty}=0.006$ Pas, $\lambda=75 \mathrm{~s}$ and $\mathrm{n}=0.3$. Furthermore, these simulations have been performed at the same flow rate $\left(63 \mathrm{~mm}^{3} / \mathrm{s}\right)$ as the experiments shown in Figure 37, at which the flow is purely laminar $(\mathrm{Re}=0.11)$. The steady laminar simulation models the piston movement using a velocity inlet. The pressure based Navier Stokes solver is able to reproduce the viscous pressure ( $\left.\mathrm{P}_{\text {viscous }}\right)$ of the experiments.

Figure 38 shows the flow velocity field in the slit entrance region for the $90^{\circ}, 45^{\circ}$ and $30^{\circ}$ entrance angles. It can be seen that the region of increased velocity increase is 
enlarged in the case of $30^{\circ}$ entrance angle in comparison with the steeper angles. Table 10 lists the maximum distance from the centre of the slit entrance to the point of $20 \%$ and $40 \%$ of the maximum velocity for the three different entrance angles, as calculated by the simulation. These regions are roughly comparable with the turquoise and yellow zones in Figure 38.

Table 10. Maximal length of the region of velocity increase derived by simulation

\begin{tabular}{cccc}
\hline $\begin{array}{c}\text { Percentage of maximal } \\
\text { velocity }\end{array}$ & $\mathbf{3 0}^{\circ}$ & $\mathbf{4 5}^{\circ}$ & $\mathbf{9 0}^{\circ}$ \\
\hline $20 \%$ & $200 \mu \mathrm{m}$ & $140 \mu \mathrm{m}$ & $80 \mu \mathrm{m}$ \\
$40 \%$ & $79 \mu \mathrm{m}$ & $57 \mu \mathrm{m}$ & $39 \mu \mathrm{m}$ \\
\hline
\end{tabular}

These results show that suspensions are exposed to a high-velocity field for longer time during the experiments with small entrance angles. The increased flow stability measured with increasing slit entrance angle is likely to be due to a decreased residence time, i.e. time of exposure to the extensional flow field.
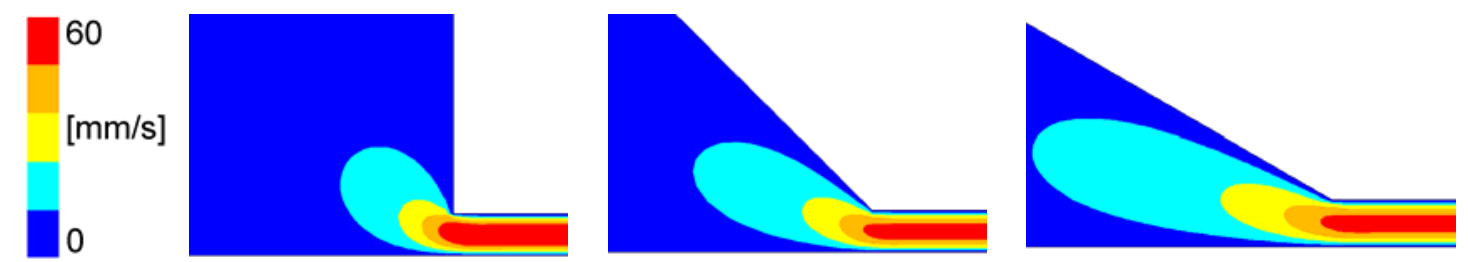

Figure 38. Comparison of computed velocity field at $\beta=90^{\circ}, 45^{\circ}$ and $30^{\circ}$ obtained with the following computation parameters: $L{ }^{`}=1.2 \mathrm{~mm}, L=0.8 \mathrm{~mm}, H=26 \mu \mathrm{m}$ and flow rate 63 $\mathrm{mm}^{3} / \mathrm{s}$.

\subsection{Influence of particle properties on the pressure increase}

In the previous sections we have shown the influence of colloidal and flow kinematics parameters on the flow stability of investigated suspensions by comparing the normalized pressure increase due to the gradual clogging of the slit entrance by the aggregates formed in the converging flow field. However, comparison of the relative flow stability of different materials based on normalized pressure increases requires an examination of the effect of particle properties and the porosity of the generated aggregates.

A characteristic feature of the PU and polychloroprene particles is the low glass transition temperature $\left(\mathrm{T}_{\mathrm{g}}=-40^{\circ} \mathrm{C}\right)$, which consequently influences the properties of the aggregates clogging the slit entrance. The soft PU and polychloroprene particles deform at room temperature to form a compact film that is impermeable to the fluid and causes a steep pressure increase. On the other hand, the PS particles are hard at room temperature $\left(\mathrm{T}_{\mathrm{g}}=95{ }^{\circ} \mathrm{C}\right)$ and form porous aggregates. As aggregates start to clog the slit, the pressure increases for the hard PS in a similar way as for the soft particles, but upon 
reaching a maximum, the pressure plateaus, due to the low flow resistance of the porous structure. It is well known that the flow resistance through porous media can be described by the Darcy's law as follows:

$$
\Delta p=\frac{1}{K} \frac{Q L_{p} \eta}{A}
$$

where $\Delta p$ is the pressure-drop across the porous medium with cross-sectional area $A$, thickness $L_{p}$ and permeability K. The permeability has the units of area and can be determined experimentally.

Molerus et al. [104] have proposed an empirical model to describe the flow resistance through porous structures by including an expression for the permeability based on the particle size $(a)$ and porosity $\left(\varepsilon_{p}\right)$ :

$$
\Delta p=22.4 \frac{1-\varepsilon_{p}}{\varepsilon_{p}^{4.5}} \frac{1}{a^{2}} \frac{Q L_{p} \eta}{A}
$$

It can be seen from equation (88) that the pressure drop across the porous structure is strongly dependent on the porosity (or the packing density of particle beads) and particle size. Large particles result in relatively large gaps between particles and the porous structure has a lower flow resistance compared to smaller particles. Hence, during the agglomeration process, the pressure drop is not as significant as for the case of smaller particles. This should be considered, comparing samples of rigid (undeformable) particles with different size.

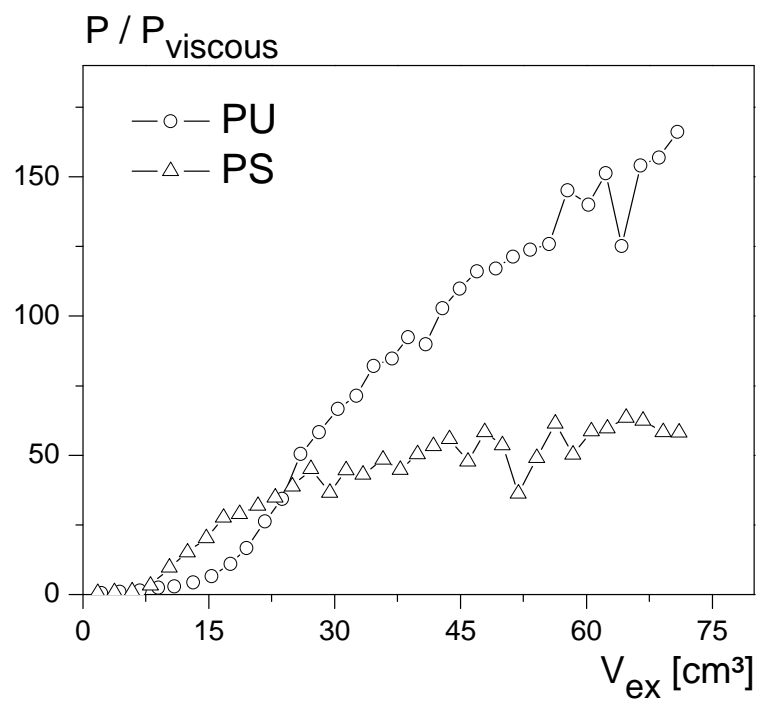

Figure 39. Comparison of normalized pressure increase due to the gradual clogging of the slit entrance by the $P U$ and PS aggregates (fine filter, $H=21 \mu \mathrm{m}, \beta=45^{\circ}$ and flow rate $=314 \mathrm{~mm}^{3} / \mathrm{s}$ )

Figure 39 shows the normalized pressure drop as a function of extruded volume for the PU and PS suspension in the case of $21 \mu \mathrm{m}$ slit height and $314 \mathrm{~mm}^{3} / \mathrm{s}$ flow rate. It can 
be seen that the pressure begins to increase earlier in the case of PS but it asymtotes after a short period of time, whereas in the case of the PU suspension the pressure increases steeply with extruded volume and reaches higher absolute values in comparison to PS. As described above, this is due to the different particle and aggregate properties at room temperature, which does not allow direct comparison of the flow stability of these two suspensions. 


\section{Conclusion and future prospects}

\subsection{Conclusion}

We have investigated the phenomenon of flow-induced aggregation in highly concentrated colloidal dispersions exposed to strongly converging flow fields. This phenomenon is relevant not only for classical technical operations like coating, pumping and filtration, but also for the application of concentrated suspensions to emerging processing technologies based on microfluidic devices. In order to investigate this phenomenon experimentally, a ring-slit device was developed that provides a strong flow contraction at the entrance of a narrow slit $(10-25 \mu \mathrm{m})$ and allows for a variation of flow kinematics (slit height, entrance angle, length of converging entrance region and flow rate) in a wide range. Aggregates are not only formed in this microfluidic set-up, but are also detected via the increase in the extrusion pressure due to the gradual clogging of the slit entrance. Charge stabilized commercial polymer dispersions (PU, PS and polychloroprene) with a primary particle size of about $100 \mathrm{~nm}$ were used as model systems having a shelf-life of at least six months. We have shown that the pressure increase is not just due to the retention of pre-existing aggregates larger than the gap height. Experiments using a concentric cylinder shear cell reveal that aggregation does not occur in pure shear flow within the straight channel, and ring-slit tests with channels of different surface properties (hydrophilic vs. hydrophobic) indicate that clogging is not induced by the deposition of particles at the channel wall. Instead, we have clearly shown that aggregation in the converging flow field at the slit entrance is the dominant effect leading to clogging of the slit. Accordingly, this phenomenon strongly depends on flow rate and, for all investigated systems, the slit clogs faster for lower flow rates. This can be explained as follows: first, interparticle contact time is important for the formation of aggregates and the aggregation probability slightly decreases with increasing flow rate; second, the dimensionless breakage number $(\mathrm{Br} \sim \dot{\gamma})$ is much larger than unity at all examined flow rates, thus aggregates may also be destroyed in the flow field. As the breakup of aggregates increases with increasing flow rate the slit clogging correspondingly decreases with the flow rate. Clogging is also more pronounced at lower entrance angles. According to simple one phase CFD calculations, we attribute the effect of slit entrance angle to a longer exposure of particles to high-velocity flow fields. The observed flow rate dependence also rules out hydrodynamic bridging as a clogging mechanism. Ring-slit experiments with differently pre-filtered dispersions and experiments with added PMMA particles in different sizes and amounts show that slit clogging is induced by the heterocoagulation of primary nano-particles with micron-sized 
objects, which are always present in commercial dispersions due to manufacturing and processing conditions. Only in the case of hetero-coagulation, the critical Peclet number necessary to overcome the repulsive potential barrier between particles is exceeded. Heterocoagulation can cause slit-clogging even if the concentration of large particles is on the order of $100 \mathrm{ppm}$ and the ratio of large particle size to gap height is proportional to 0.1 . On the other hand, heterocoagulation can be strongly influenced by the colloidal properties of the primary particles and strong differences in the ring slit tests are observed for the PU, PS and polychloroprene dispersions under otherwise similar test conditions. Slit-clogging can be suppressed even in the presence of large micron-sized particles by appropriate stabilization of the primary particles either by the addition of a sufficient amount of surfactant providing full particle coverage or through an appropriate reduction in the ionic strength, which increases the range of electrostatic repulsion.

This work provides a sound experimental basis that clearly identifies hetero-coagulation as the elementary step in the clogging of microchannels. However, we are still far from providing a quantitative interpretation and understanding of such complex systems as the highly concentrated commercial dispersions exposed to strongly converging flow fields that have been investigated here. Despite this lack of quantitative theories, we can provide different strategies to improve processing stability: first, reduce the amount of micron-sized particles by using appropriate manufacturing conditions; second, improve the colloidal stability of the primary nano-particles through appropriate surfactants or surface charge density modification. Finally, details of the flow kinematics also play a crucial role and appropriate design of processing equipment (gap dimensions, contraction angle) can provide stability. On the other hand, when clogging or aggregation is desired the addition of a small amount of micron-sized particles to a nano-particulate dispersion in combination with an appropriate design of the flow field might offer new options in solid-liquid separation technology.

\subsection{Zusammenfassung}

Im Rahmen dieser Arbeit wurde die strömungsinduzierte Agglomeration von hochkonzentrierten Dispersionen in stark konvergenten Strömungen untersucht. Dieses Phänomen ist relevant nicht nur für klassische Prozesse wie Filtration, Pumpen und die industrielle Beschichtungsverfahren sondern auch für die Entwicklung neuer Verfahren in der Mikrofluidik. Um dieses Phänomen experimentell zu untersuchen wurde der sogenannte Ringschlitz-Test entwickelt, welche die Realisierung kleiner Spalthöhen (10-25 $\mu \mathrm{m})$ bei gleichzeitig großer Querschnittsfläche ermöglicht und eine starke Kontraktion der Strömung gewährleistet. Durch Variation der Spalthöhe, des Volumenstroms und des Einlaufwinkels kann die Strömungskinematik in einem weiten Bereich variiert und an den jeweils relevanten technischen Prozess angepasst werden. Bei diesem 
microfluidischen Aufbau wird die Agglomeartion hervorgerufen und direkt durch den damit einhergehenden Druckanstieg zeitaufgelöst verfolgt. Drei kommerzielle Polymerdispersionen (PU, PS und polychloroprene), mit einer mittleren Partikelgröße von ca. $100 \mathrm{~nm}$, wurden im Rahmen dieser Studie als Modellsysteme untersucht. Der Fokus liegt auf Dispersionen mit repulsiv wechselwirkenden Partikeln, die hohe Lagerstabilität besitzen. Für alle untersuchten Dispersionen wurde, trotz der hohen Lagerstabilität, Verstopfen der Ringschlitz-Düse beobachtet, jedoch bei unterschiedlichen Messbedingungen. Die Ursachen für das Verstopfen von Mikrokanälen können sehr unterschiedlich sein. Neben der Agglomeration können die Ablagerung von Partikeln an die Wand oder Brückenbildung von Partikeln die gleichzeitig in dem Mikrokanal einlaufen, Ursachen für das Blockieren des Spaltes sein. Im Rahmen dieser Arbeit wurden die oben genannten Mechanismen durch gezielte Experimente ausgeschlossen. RingschlitzTests mit unterschiedlich beschichteten Spaltoberflächen (hydrophil vs. hydrophob) haben gezeigt dass Partikel-Wand Wechselwirkungen irrelevant für das Verstopfen des Spaltes sind. Es wurde weiterhin anhand von Experimenten in der reinen Scherströmung zwischen zwei konzentrischen Zylindern gezeigt, dass die Spalt-Verstopfung nicht auf Aggregatbildung in der Scherströmung innerhalb des Spaltes zurückzuführen ist. Stattdessen zeigen unsere experimentellen Untersuchungen, dass Aggregation in der stark konvergenten Strömung am Kanaleintritt das Verstopfen des Spaltes verursacht. Dementsprechend lässt sich die strömungsinduzierte Agglomeration stark durch die Scherrate und damit durch den Volumenstrom beeinflussen. Für alle untersuchen Dispersionen wurde die gleiche Volumenstromabhängigkeit beobachtet, mit abnehmendem Volumenstrom nimmt das Verstopfen der Ringschlitz-Düse zu. Diese Volumenstromabhängigkeit schließt das Spalt-Verstopfen durch hydrodynamische Brückenbildung am Kanaleintritt aus und lässt sich durch das Wechselspiel zwischen den hydrodynamischen und den kolloidalen Kräfte erklären. Dabei ist auch entscheidend wie lange sich die Partikel innerhalb der Reichweite der attraktiven Wechselwirkungen aufhalten. Mit zunehmender Scherrate nimmt die Kontaktzeit ab und führt zur Reduzierung der Kollisionseffizienz. Die Kollisionseffizienz bei elektrostatisch stabilisierten Dispersionen hängt auch davon ab, ob die Scherkraft die elektrostatische Abstoßungskraft zwischen Partikeln überwinden kann. Demzufolge begünstigen hohe Scherraten die Agglomeration von elektrostatisch stabilisierten Dispersionen. Andererseits können die hydrodynamischen Kräfte im Bereich hoher Schergeschwindigkeiten die van der Waals-Bindungskräfte überwinden und damit Bruch von Agglomeraten verursachen. Die Kräfteverhältnisse von hydrodynamischen zu den Abstoßungs- und Anziehungskräften für die untersuchte Dispersionen und Volumenströme zeigen, dass die hydrodynamischen Kräfte im Kanaleinlauf sowohl die Elektrostatische Abstoßungsbarriere $\left(\mathrm{Pe}_{\mathrm{c}}>1\right)$, als auch die van der Waals-Bindungskräfte $(\mathrm{Br}>1)$ überwinden können. Folglich wird die Aggregatgrössenverteilung von einem dynamischen Gleichgewicht zwischen Aggregation und Bruch bestimmt. Dabei nimmt die maximale Aggregatgröße mit dem Volumenstrom ab und da 
die Spalt-Verstopfung durch Agglomerate mit einer bestimmten Größe verbunden ist, nimmt der Druckanstieg mit dem Volumenstrom auch ab.

Das Verstopfen von Mikrokanälen durch Agglomeration in der konvergenten Strömung am Kanaleintritt kann auch durch den Einlaufwinkel beeinflusst werden. Es wurde experimentell festgestellt, dass das Verstopfen des Spaltes bei flachen Winkeln ausgeprägter ist als bei den steileren Winkeln. Der Einfluss des Winkels bezieht sich auf die Verweilzeit der Partikeln. Mit Hilfe von CFD-Simulationen wurde gezeigt, dass die Verweilzeit der Partikel in der Dehnströmung umso länger ist je flacher der Einlaufwinkel ist. Mit zunehmender Verweilzeit nimmt die erreichbare Aggregatgröße zu und es kann mit hoher Wahrscheinlichkeit zum Verstopfen des Spaltes kommen.

Ringschlitz-Experimente mit durch Siebe unterschiedlicher Maschenweite vorfiltrierten Dispersionen sowie Experimente mit Zugabe von PMMA Partikeln definierter Größe und Menge haben gezeigt, dass das Spalt-Verstopfen durch Heterokoagulation zwischen den Primärnanopartikeln und Mikrometer großen Verunreinigungen verursacht wird. Dabei handelt es sich um Partikel und Agglomerate in Mikrometer-Bereich, die sich bei den kommerziellen Dispersionen aufgrund der Herstellungs- und Verarbeitungsbedingungen nicht vermeiden lassen. Es sollte darauf hingewiesen werden, dass die kritische Peclet-Zahl $\left(\mathrm{Pe}_{\mathrm{c}}\right)$, bei welcher die hydrodynamische Kräfte die elektrostatische Abstoßungsbarriere überwinden können, nur im Falle von einer Heterokoagulation überschritten wird. Bereits geringe Konzentrationen (100 - 1000 ppm) dieser größeren Partikeln/Agglomeraten und kleine Größenverhältnisse (Partikeldurchmesser zu Spalthöhe $\approx 0.1$ ) können Verstopfen von Mikrokanälen durch Heterokoagulation in der konvergenten Strömung am Kanaleintritt hervorrufen. Außerdem wurde gezeigt, dass die Heterokoagulation durch die kolloidalen Eigenschaften der Primärnanopartikeln stark beeinflusst werden kann. Die Stabilisierung der PU-, PS- und polychloroprene Partikeln wurde systematisch variiert durch gezielte Einstellung der Ionenstärke und durch Zugabe von höher molekularen und polymeren Tensiden. Die Ergebnisse zeigen, dass die Stabilität aller untersuchten Dispersionen sich durch Zusatz von Tensiden und Reduzierung der Ionenstärke entscheidend verbessert und damit das Verstopfen von Mikrokanälen verhindert werden kann, trotz des Vorhandenseins großer Partikel/Agglomerate.

Diese experimentelle Arbeit liefert solide Beweise, die auf die Heterokoagulation in Dehnströmungen als grundlegender Mechanismus für das Verstopfen von Mikrokanälen hindeutet. Dennoch sind wir weit von einer quantitativen Interpretation und Verständnis komplexer Systeme, wie die untersuchten kommerziellen hoch-konzentrierten Dispersionen, die stark konvergenten Strömungen ausgesetzt sind. Trotz unzureichender Theorie zur quantitativen Beschreibung dieses Phänomens, können anhand der gewonnenen experimentellen Erkenntnisse, technisch umsetzbare Strategien zur Vermeidung der Agglomeration entwickelt werden: ersten, man reduziert die Größe und Konzentration 
der Mikrometer großen Verunreinigungen durch anpassen der Produktions- und Verarbeitungsbedingungen; zweitens, man verbessert die Stabilisierung der Primärpartikeln durch gezielte Einstellung der Ionenstärke und durch Zugabe von geeigneten Tensiden. Eine wichtige Erkenntnis dieser Arbeit ist, dass die Strömungskinematik eine entscheidende Rolle spielt und dass die Agglomeration in Dehnströmungen durch geeignete Konstruktion von Verarbeitungs- und Applikationsgeräte (Spalthöhe, Einlaufwinkel) reduziert werden kann. Andererseits, wenn die Agglomeration und das Verstopfen von Mikrokanälen gewünscht sind, bietet die Zugabe von kleinen Mengen an Mikrometer großen Teilchen neue Optionen für die fest-flüssig Trennung an.

\subsection{Future prospects}

The knowledge on flow induced aggregation in converging flow filed and the process of clogging of microchannels should be utilized in the development of technological processes, for example in the field of printed electronics, where aggregation of monometallic particles can cause clogging of the inkjet nozzle and affect the printing performance. Preliminary experiments using zinc oxide dispersions showed that the clogging of inkjet nozzles correlates with the results from the ring-slit test. It should be examined in a future study whether the same strategies can be applied to prevent clogging of the inkjet nozzles as found from the laboratory scale study on polymer dispersions. For this purpose, the inkjet printing performance and processing stability should be investigated systematically using inorganic nano-dispersions and the correlation between ring-slit and inkjet printer experiments should be verified for a wide range of process parameters and dispersion properties.

Furthermore, the monometallic dispersions offer the advantage of controlled variation of the size and amount of pre-existing aggregates by centrifugation and thus avoiding the problem of poor filtration efficiency limited by the elongated form of the aggregates present in the polymer dispersions investigated here. 


\section{$6 \quad$ Appendix}

\subsection{Complementing experimental results}

\subsubsection{High-shear viscosity of investigated samples}

The high shear viscosity of the highly concentrated polymer dispersions investigated in this work was measured using a capillary viscometer (Rheograph 6000, Göttfert Werkstoff-Prüfmaschinen GmbH, Germany). The samples were extruded from a reservoir through a capillary die at a constant volumetric flow rate which is controlled by the piston velocity. The resulting pressure drop is detected by a transducer mounted above the entrance of the capillary. Hence the measured pressure drop includes not only the pressure loss along the capillary but also the entrance pressure loss due to the convergence of the flow at the capillary entrance.

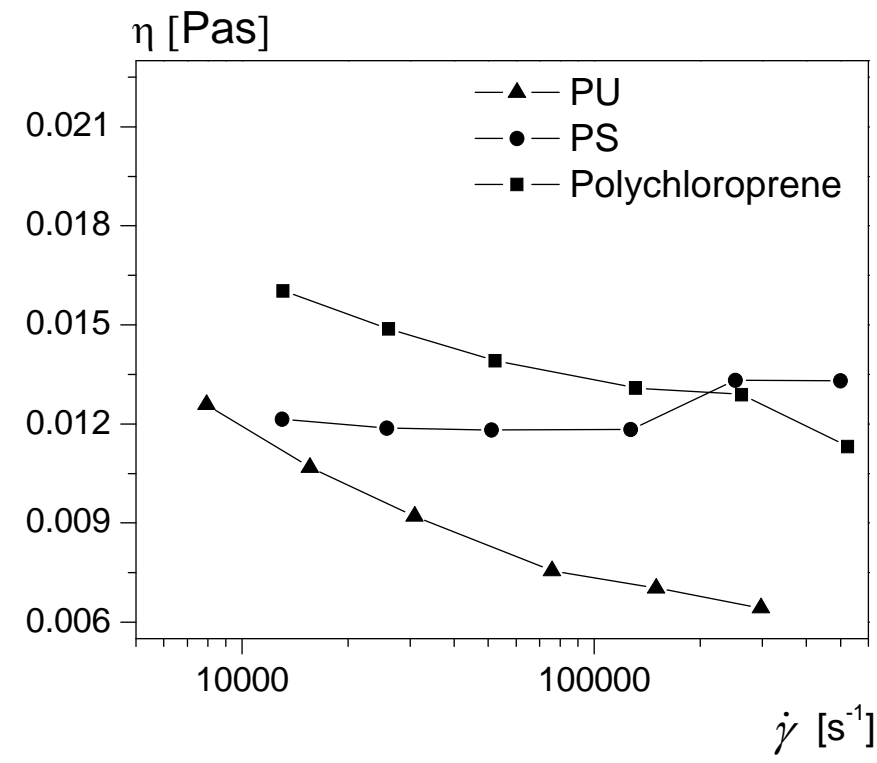

Figure 40. High shear viscosity of the PU, PS and polychloroprene suspension

Assuming Newtonian flow behaviour the apparent wall shear rate and apparent wall shear stress can be calculated using equation (73). In order to get the true shear stress Bagley's correction has been applied. For this purpose we extruded the samples through capillary dies with the same radius $(0.5 \mathrm{~mm})$ but different length $(40 \mathrm{~mm}, 20 \mathrm{~mm}, 10$ $\mathrm{mm}$ ). For constant shear rate the measured pressure drop is plotted versus the capillary length to capillary radius ratio (Bagley plot) and the entrance pressure loss is obtained from linear extrapolation of the data points to the intercept with the pressure-axis. The true shear rate at the wall was obtained using the Weissenberg-Rabinowitsch correction 
for the non-Newtonian velocity profile. Considering these corrections the true viscosity is computed and plotted versus shear rate in Figure 40.

\subsubsection{Examination of the sensor calibration for the AccuSizer 780 AD}

As discussed in section 3.1.3.2.1, the average size of polystyrene standard measured by AccuSized 780 AD differs from the certified particle diameter. In order to examine the deviation in the particle size for a large particle size range we measured polystyrene standards of different diameter (d).

Table 11. Comparison of certified size standards with sizing results in extinction and summation range obtained using AccuSizer 780 AD.

\begin{tabular}{|c|c|c|c|c|c|}
\hline \multicolumn{2}{|c|}{ certified size standard } & \multicolumn{2}{c|}{$\begin{array}{c}\text { measured size in extinction } \\
\text { range }\end{array}$} & \multicolumn{2}{c|}{$\begin{array}{c}\text { measured size in summa- } \\
\text { tion range }\end{array}$} \\
\hline $\mathrm{d}[\mu \mathrm{m}]$ & deviation [\%] & $\mathrm{d}[\mu \mathrm{m}]$ & $\begin{array}{c}\text { deviation from } \\
\text { certified d [\%] }\end{array}$ & $\mathrm{d}[\mu \mathrm{m}]$ & $\begin{array}{c}\text { deviation from } \\
\text { certified d [\%] }\end{array}$ \\
\hline 1.00 & \pm 3.00 & - & - & 0.96 & -4.00 \\
\hline 2.22 & \pm 2.70 & 2.47 & 11.26 & 2.39 & 7.66 \\
\hline 4.62 & \pm 2.38 & 4.46 & -3.46 & 4.57 & -1.08 \\
\hline 10.37 & \pm 1.35 & 11.05 & 6.56 & 11.44 & 10.32 \\
\hline 19.66 & \pm 1.02 & 21.83 & 11.04 & 20.71 & 5.34 \\
\hline 29.00 & \pm 1.03 & 31.38 & 8.21 & 31.89 & 9.97 \\
\hline 39.70 & \pm 1.01 & 45.12 & 13.65 & 44.08 & 11.03 \\
\hline 50.70 & \pm 0.99 & 56.61 & 11.66 & 54.71 & 7.91 \\
\hline 76.80 & \pm 0.65 & 85.17 & 10.90 & 79.82 & 3.93 \\
\hline
\end{tabular}

Table 11 shows the sizing results of the used polystyrene standards (BS-partikel, Germany) measured in extinction and summation range. It can be seen that almost all of the polystyrene sizing results are higher than expected and the maximum difference between expected and measured diameter is about $14 \%$. The expected size and deviation values were provided by the standards manufacture and were also determined via particle counting instrument on the basis of the SPOS method ("Syringe", Markus Klotz, Germany). It is evident from Table 11 that sensor type (summation versus extinction) also impacts the sizing results, but no trend can be recognized.

\subsubsection{Effect of pre-filtration}

Figure 41 shows the effect of pre-filtration on the slit clogging phenomenon for the PU suspension at different flow rates $\left(63\right.$ and $\left.1570 \mathrm{~mm}^{3} / \mathrm{s}\right)$. These results are similar to those at $314 \mathrm{~mm}^{3} / \mathrm{s}$ ( Figure 25). Interpretation of the effect of pre-filtration on the flow induced aggregation is given in section 4.2. 

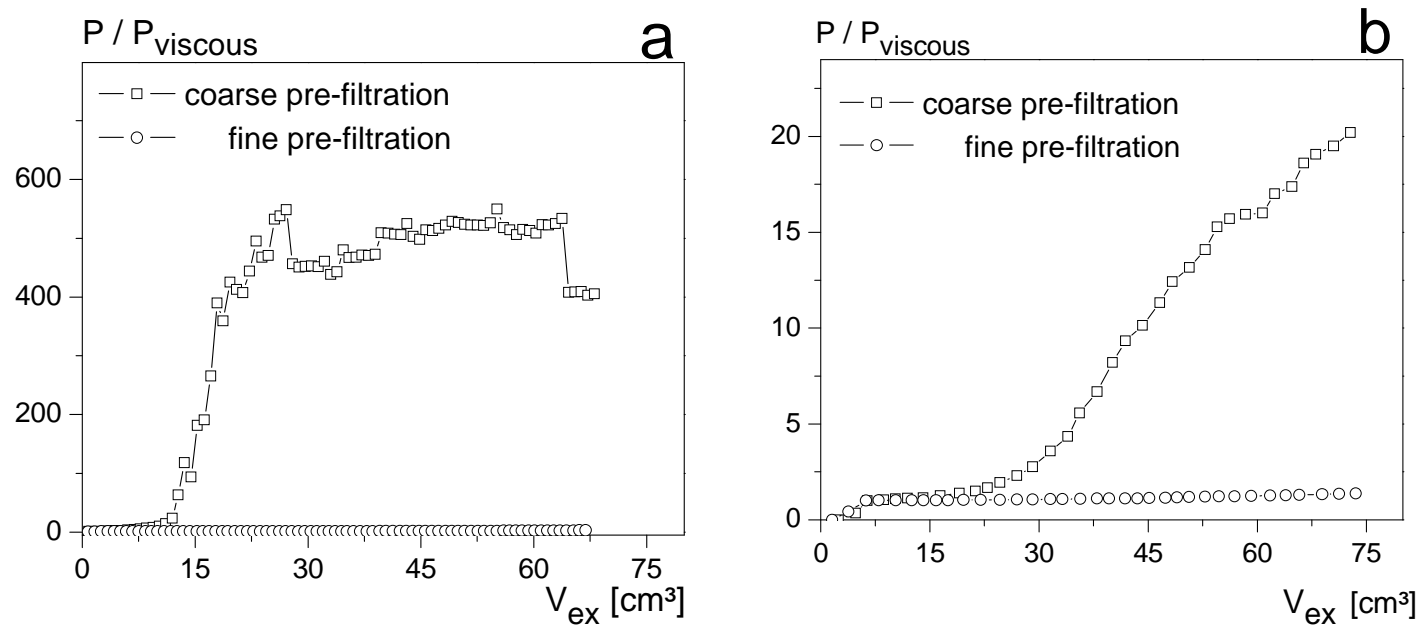

Figure 41. Effect of pre-filtration on the flow induced aggregation of PU dispersion ( $\phi=0.4$, $\left.H=21 \mu \mathrm{m}, \beta=45^{\circ}\right)$ at $63 \mathrm{~mm}^{3} / \mathrm{s}($ a $)$ and at $1570 \mathrm{~mm}^{3} / \mathrm{s}(\mathrm{b})$

\subsubsection{Filter cake formation of PMMA particles}

The effect of small amount of micron-sized PMMA particles on the flow induced aggregation of the PU dispersion has been discussed in section 4.2.2. It has been shown that the slit clogging is due to hetero-coagulation between the PMMA and PU particles. Figure 42a shows the normalized pressure drop as a function of extruded volume for the fine pre-filtered PU dispersion containing $1 \%$ PMMA particles. At this concentration of the PMMA particles the pressure increase is due to filter cake formation as evidenced by the electron micrograph of the coagulum retained at the slit entrance (Figure 42b). These results can be explained by PMMA particle aggregation due to the increased probability of collision, which dominates the hetero-coagulation mechanism. Note that the PMMA particles used in these experiments are maximum by factor of 2 smaller in diameter than the slit height and doublet or triplets could be sufficient to clog the slit. Ring-slit test of the PMMA particles dispersed in aqueous glycerin solution revealed a pressure increase, indicating slit clogging by the PMMA particles.

The porous nature of the filter cake at the slit entrance can explain the effect of PMMA particle size on the pressure increase shown in Figure 42a. Larger particles result in higher porosity of the PMMA filter cake and hence a lower flow resistance compared to smaller particles. The influence of porosity and particles size on the pressure increase is discussed in section 4.6 in more detail. 

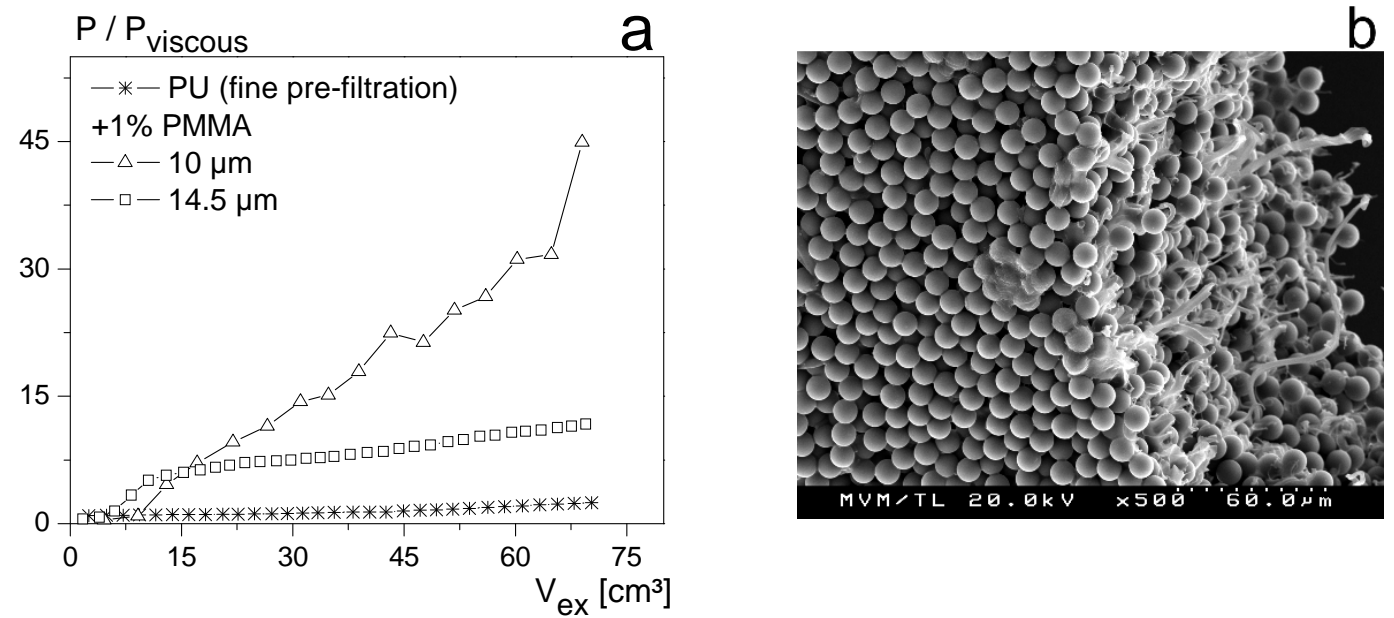

Figure 42. Comparison of the normalised pressure increase due to the gradual clogging of the slit entrance by monodispersed PMMA particles of different size (a) and electron microfraph of the coagulum after the ring-slit test of fine pre-filtered PU dispersion with 1\% PMMA particles

\subsubsection{Effect of surfactant on flow induced aggregation}

The influence of surfactant on the flow induced aggregation for the mixture of fine prefiltered PU dispersion and 100 ppm PMMA particles (see Figure 43a for 10 $\mu \mathrm{m}$ PMMA and Figure $43 \mathrm{~b}$ for 14.5 PMMA) is similar to the results for the coarse pre-filtered PU dispersion (Figure 31). The stabilizing effect of the SDS surfactant on the flow stability of the PU and PS dispersion in the case of $30^{\circ}$ entrance angle and $26 \mu \mathrm{m}$ slit height is shown in Figure 44.
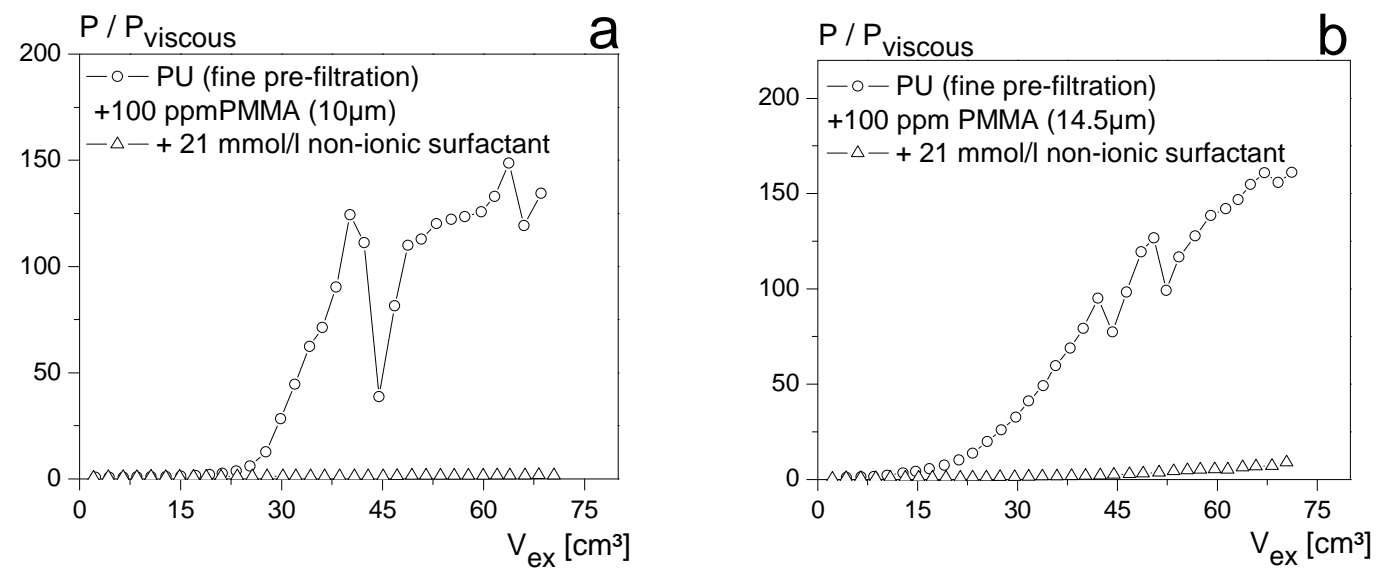

Figure 43. Effect of non-ionic polymeric surfactant on the flow stability of PU(fine pre filtration) with $100 \mathrm{ppm}$ PMMA particles of $10 \mu \mathrm{m}$ (a) and $14.5 \mu \mathrm{m}$ (b)in diameter $\left(H=21 \mu \mathrm{m} \beta=45^{\circ}\right.$, and flow rate $=314 \mathrm{~mm}^{3} / \mathrm{s}$ ) 

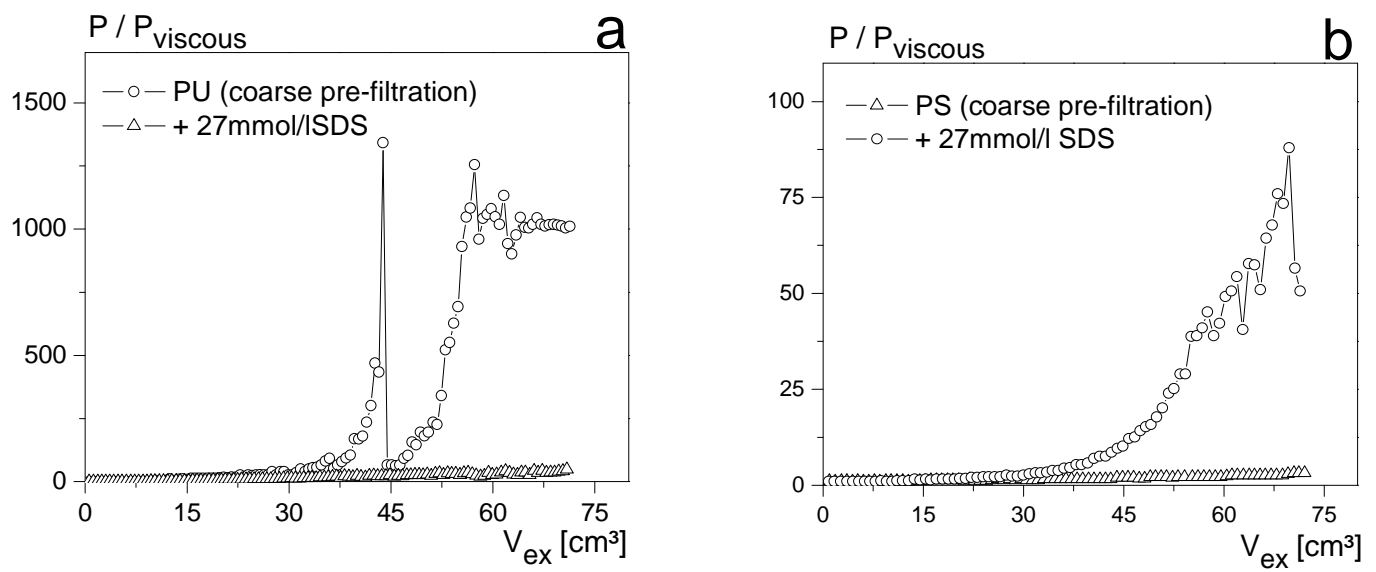

Figure 44. Effect of SDS surfactant on the flow stability of $P U$ (coarse pre filtration, $H=26 \mu \mathrm{m}$ $\beta=30^{\circ}$, and flow rate $=23 \mathrm{~mm}^{3} / \mathrm{s}$ ) (a) and PS (coarse pre filtration, $H=26 \mu \mathrm{m} \beta=30^{\circ}$, and flow rate $\left.=63 \mathrm{~mm}^{3} / \mathrm{s}\right)(\mathrm{b})$

\subsubsection{Effect of particle concentration}

The effect of PU particle concentration on particle retention for a $21 \mu \mathrm{m}$ slit is shown in Figure $45 \mathrm{a}$ for the flow rate of $63 \mathrm{~mm}^{3} / \mathrm{s}$ and in Figure $45 \mathrm{~b}$ for $1570 \mathrm{~mm}^{3} / \mathrm{s}$. The results are similar to those at $314 \mathrm{~mm}^{3} / \mathrm{s}$ volumetric flow rate (Figure 30 ), reported and discussed in section 4.2.3.
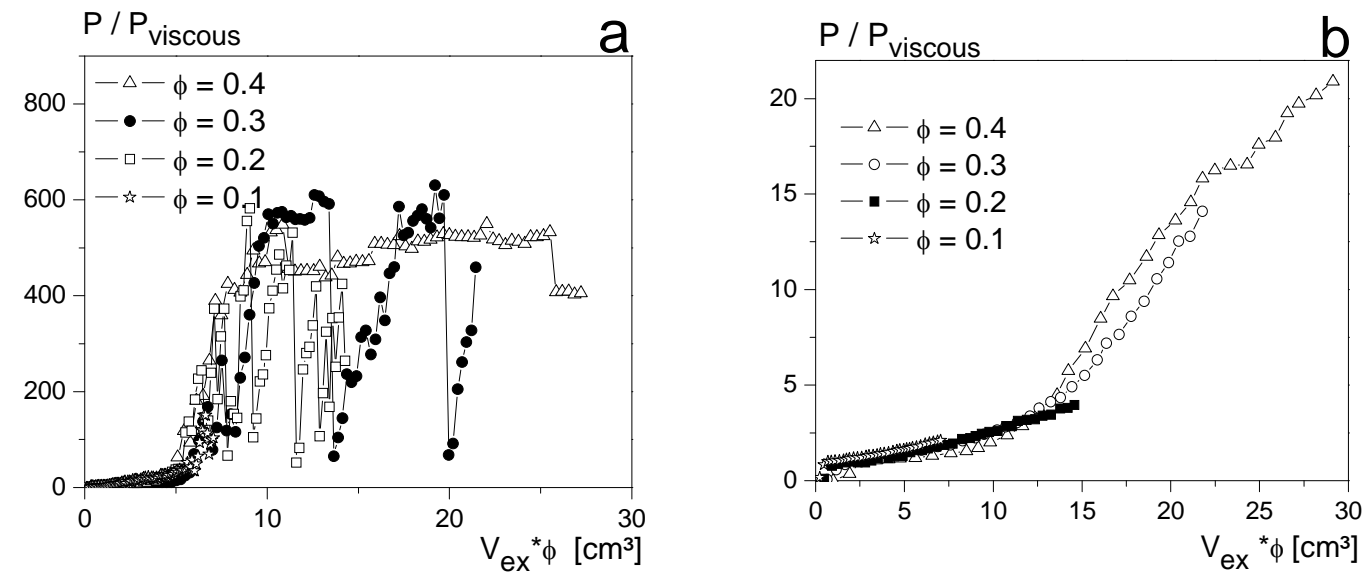

Figure 45. Effect of volume fraction on the clogging phenomenon for the coarse prefiltered $P U$ dispersion $\left(H=21 \mu \mathrm{m}\right.$ and $\left.\beta=45^{\circ}\right)$ at flow rate of $63 \mathrm{~mm}^{3} / \mathrm{s}$ (a) and at 1570 $\mathrm{mm}^{3} / \mathrm{s}(b)$ 


\subsection{List of symbols and abbreviations}

Latin symbols

\begin{tabular}{|c|c|c|c|}
\hline a & Particle radius & $\mathrm{K}$ & Permeability \\
\hline$a_{\text {eff }}$ & Effective hard sphere radius & $\mathrm{k}_{\mathrm{B}}$ & Boltzmann constant \\
\hline$A_{H}$ & Hamaker constant & $\mathrm{k}_{\mathrm{fl}}$ & Flow kinematics coefficient \\
\hline A & Area & $\mathrm{k}_{\mathrm{r}}$ & Rate constant of rapid aggrega- \\
\hline $\mathrm{A}^{+}$ & Upstream interception area & & tion \\
\hline$A_{x_{3}>0}^{+}$ & Upstream interception area contain- & $\mathrm{k}_{\mathrm{s}}$ & $\begin{array}{l}\text { Rate constant of slow aggrega- } \\
\text { tion }\end{array}$ \\
\hline B & ing the point $x_{3}$ & 1 & Dimensionless interparticle sep- \\
\hline $\begin{array}{l}\mathrm{B} \\
\mathrm{Br}\end{array}$ & Gap width & & aration \\
\hline $\mathrm{b} 1$ & Breakage number & $\mathrm{L}$ & Gap length \\
\hline & Colid & $\mathrm{L}^{\prime}$ & Entrance length \\
\hline $\begin{array}{c}\mathrm{c}_{\mathrm{s}} \\
\mathrm{d}\end{array}$ & $\begin{array}{l}\text { Solld content } \\
\text { Particle diame }\end{array}$ & $\mathrm{L}_{\mathrm{p}}$ & Thicknes \\
\hline $\begin{array}{l}\mathrm{a} \\
\mathrm{d}_{\mathrm{ch}}\end{array}$ & Channel diameter & $\mathrm{N}_{\mathrm{f}}$ & Flow number \\
\hline $\mathrm{d}_{50}$ & Median diameter & $\mathrm{N}_{\mathrm{r}}$ & Repulsion number \\
\hline $\begin{array}{l}\mathrm{C}_{50} \\
\mathrm{D}\end{array}$ & Diffusion coefficient & $\mathrm{N}_{12}$ & Strength of the vdW attraction \\
\hline $\begin{array}{l}\mathrm{D} \\
\mathrm{D}_{0}\end{array}$ & $\begin{array}{l}\text { Dirmusion coemint } \\
\text { Bare diffusion coefficient }\end{array}$ & & relative to Born repulsion \\
\hline $\mathbf{D}_{0}$ & Dare umusion coemintert & $\mathrm{n}$ & Number concentration \\
\hline$D_{s}$ & Short-time selt diffusivity & $\mathrm{n}_{\infty}$ & Bulk number concentration \\
\hline$\widetilde{\mathrm{E}}$ & deformation rate tensor & $\mathrm{n}_{0}$ & Initial number concentration \\
\hline$\vec{e}$ & Unity vector & $\mathrm{n}_{\text {tot }}$ & Total number concentration \\
\hline e & Elementary charge & $\vec{n}$ & Normal unity vector \\
\hline G & Gibbs free energy & $\mathrm{n}^{\prime}$ & Exponent \\
\hline$G_{\infty}^{\prime}$ & High frequency elastic modulus & $\mathrm{P}$ & Aggregation probability \\
\hline $\mathrm{H}_{\mathrm{e}}$ & Enthalpy & $\mathrm{P}_{\text {viscous }}$ & Viscous pressure \\
\hline $\mathrm{H}$ & Gap height & $\mathrm{Pe}$ & Peclet number \\
\hline $\mathrm{h}_{\mathrm{c}}$ & critical interparticle separation & & \\
\hline $\mathrm{h}$ & Interparticle separation & $\mathrm{Pe}_{\mathrm{c}}$ & Critical Peclet number \\
\hline $\mathrm{I}$ & Ionic strength & $\Delta \mathrm{p}$ & Pressure drop \\
\hline$i$ & $\begin{array}{l}\text { Coefficient accounting for the flow } \\
\text { kinematics }\end{array}$ & $\mathrm{Q}$ & Volumetric flow rate \\
\hline $\mathrm{J}^{0}$ & Number of collisions & $\mathrm{Q}^{+}$ & Volumetric flow rate into the \\
\hline $\mathrm{J}_{\mathrm{ij}}$ & $\begin{array}{l}\text { Number of collisions between } \\
\text { spheres of type } j \text { and type } i\end{array}$ & & collision sphere \\
\hline
\end{tabular}




\begin{tabular}{|c|c|c|c|}
\hline $\mathrm{R}$ & $\begin{array}{l}\text { Radius of rheometer sample cham- } \\
\text { ber }\end{array}$ & \multicolumn{2}{|c|}{ Greek symbols } \\
\hline $\mathrm{R}_{\mathrm{a}}$ & Average surface roughness & $\alpha$ & Collision efficiency \\
\hline $\operatorname{Re}$ & Reynolds number & $\beta$ & Slit entrance angle \\
\hline $\mathrm{r}$ & Center-to-center separation distance & $\gamma$ & Reduced surface potential \\
\hline $\mathrm{r}_{\mathrm{ij}}$ & Collision radius & $\dot{\gamma}$ & Shear rate \\
\hline$\vec{r}$ & Position vector & $\dot{\gamma}_{a p p}$ & Apparent shear rate \\
\hline $\mathrm{S}$ & Entropy & & \\
\hline St & Stokes number & $\dot{\gamma}_{c}$ & Critical shear rate \\
\hline s & Radius of circle & $\delta$ & Stern layer \\
\hline $\mathrm{T}$ & Temperature & $\varepsilon$ & Permittivity \\
\hline $\mathrm{T}_{\mathrm{g}}$ & Glass transition temperature & $\varepsilon_{\mathrm{r}}$ & Relative permittivity \\
\hline $\bar{T}$ & Mean residence time in the collision & $\varepsilon_{0}$ & Vacuum permittivity \\
\hline & sphere & $\varepsilon_{\mathrm{p}}$ & Porosity \\
\hline $\mathrm{T}_{\text {contact }}$ & Contact time & $\zeta$ & Zeta-potential \\
\hline $\mathrm{T}_{\mathrm{c}}$ & Critical contact time & $\eta$ & Dynamic viscosity \\
\hline $\mathrm{t}$ & Time & $\eta_{\text {app }}$ & Apparent dynamic viscosity \\
\hline $\operatorname{tr}$ & Trace & & Zero shear viscosity \\
\hline $\mathrm{t}_{\text {contact }}$ & $\begin{array}{l}\text { Time spent by two particles in im- } \\
\text { mediate vicinity }\end{array}$ & $\begin{array}{l}\eta_{0} \\
\kappa^{-1}\end{array}$ & $\begin{array}{l}\text { Zero shear viscosity } \\
\text { Debye length }\end{array}$ \\
\hline$t_{\text {drainage }}$ & Time required for drainage & $\lambda$ & Characteristic wavelength of \\
\hline $\mathrm{U}$ & Electrophoretic mobility & & dispersion interaction \\
\hline $\mathrm{V}_{\mathrm{s}}$ & Volume of collision sphere & $\mu$ & Viscosity of the liquid medium \\
\hline $\mathrm{v}$ & Piston speed & $\rho$ & Particle density \\
\hline$\vec{v}$ & Flow velocity & $\rho_{\mathrm{fl}}$ & Fluid density \\
\hline $\bar{v}$ & Average velocity & $\sigma$ & Suspension conductivity \\
\hline W & Stability ratio & $\sigma_{\mathrm{e}}$ & Serum conductivity \\
\hline $\mathrm{X}$ & Coefficient & $\sigma_{\mathrm{p}}$ & Particle conductivity \\
\hline $\mathrm{x}$ & Distance & $\tau$ & Characteristic \\
\hline $\mathrm{y}_{\mathrm{s}}$ & Center of mass of semi-circle & & time \\
\hline$z_{i}$ & Valence of ionic species $i$ & $\tau_{\mathrm{app}}$ & apparent shear stress \\
\hline
\end{tabular}


$\phi \quad$ Volume fraction

$\phi_{\max } \quad$ Maximum packing fraction

$\phi_{\max , \mathrm{hs}} \quad$ Maximum packing fraction of hard

sphere suspensions

$\Psi_{0} \quad$ Surface potential

$\Psi_{\delta} \quad$ Stern potential

$\Psi_{\mathrm{vdW}} \quad$ Van der Waals potential

$\Psi_{\mathrm{el}} \quad$ Electrical potential

$\Psi_{\text {Born }} \quad$ Born potential

$\Psi_{\mathrm{b}} \quad$ Interparticle bonding energy

$\Psi_{\max } \quad$ Potential energy barrier

$\Psi_{\text {min }} \quad$ Primary potential minimum

$\widetilde{\Omega} \quad$ Vorticity tensor

$\omega$ Frequency of oscillation 


\section{References}

[1] R. Ettl, V. Schädler, N. Willenbacher, "Runnability and flow-induced aggregation of paper coating suspensions”, Nordic Pulp and Paper Res. J., 15 (2000) 509

[2] P. C. Hiemenz, "Principles of colloid and surface chemistry" Marcel. Dekker, Inc., New York, 1997

[3] O. Stern „Zur Theorie der elektrolytischen Doppelschicht“, Elektrochemie, 30 (1924) 508

[4] J. Gregory, "Particle in water: properties and precesses", Taylor \& Francis Group (2006)

[5] A. L. Loeb, J. Th. G Overbeek, P. H. Wiersema, "The electrical double layer around a spherical colloid particle" M.I.T. Press (1961)

[6] D. J. Shaw, „Introduction to colloid and surface chemistry”, 4rd ed., ButterworthHeinemann (1992)

[7] M. Elimelech, J. Gregory, X. Jia, R.A. Williams, "Particle deposition and aggregation: measurements, modelling and simulation”, Butterworth-Heinemann. (1998)

[8] R. J. Hunter, "Zeta potential in colloid science: principles and applications", Academic Press, London (1981)

[9] D. C. Henry, "The cataphoresis of suspended particles. Part I. The equation of cataphoresis", Proc. Roy. Soc. A, 133 (1931) 106

[10] J. Lyklema, "Fundamentals of interface and colloid science; 2) Solid-liquid interfaces" Academic Press London, 1995

[11] P. H. Wiersema, A. L. Loeb, J. T. G. Overbeek, "Calculation of electrophoretic mobility of a spherical colloid particle" J. Colloid Interface Sci., 22 (1966) 78

[12] R. W. O’Brien, L. R. White, "Electrophoretic mobility of a spherical colloidal particle" J. Chem. Soc., Faraday Trans. 2, 74 (1978) 1607

[13] H. C. Hamaker, "The London-van der Waals attraction between spherical particles", Physica, 4 (1937) 1058

[14] W.B. Russel, D.A. Saville, W.R. Schowalter, "Colloidal Dispersions", Cambridge University Press, Cambridge, 1989.

[15] J. Gregory, "The calculation of Hamaker constants", Adv. Colloid Interface Sci., 2 (1969) 396 
[16] J. N. Israelachvili, "Intermolecular and surface forces", Academic Press: London, $\mathrm{UK}, 1992$

[17] E. J. W. Verwey, J. Th. G. Overbeek "Theory of the stability of lyophobic colloids" Elsevier, Amsterdam (1948)

[18] J. Gregory, "Interaction of unequal double-layers at constant charge", J. Colloid Interface Sci., 51 (1975) 44

[19] D. F. Evans, H. Wennerström, "Colloidal domain where physics, chemistry, biology, and technology meet", Weinheim: VCH, New York (1994)

[20] R. Hogg, T. W. Healy, D.W. Fuerstenau, "Mutual coagulation of colloidal dispersions", Trans. Faraday Soc., 62 (1966) 1638

[21] J. E. Sader, S. L. Carnie and D. Y. C. Chan, "Accurate analytic formulas for the double layer interaction between spheres” J. Colloid Interface Sci., 171 (1995) 46

[22] B.V. Derjaguin, L. D.Landau, "Theory of the stability of strongly charged lyophobic sols and of the adhesion of strongly charged particles in solutions of electrolytes.", Acta Phys. Chim. URSS, 14 (1941) 733

[23] D. L. Feke, N. D. Prabhu, J. A. Mann Jr, J. A. Mann, “A formulation of the shortrange repulsion between spherical colloidal particles.”, J. Phys. Chem., 88 (1984) 5735.

[24] D. H. Napper, A. Netschey, "Studies of the steric stabilization of colloidal particles”, J. Colloid Interface Sci., 37 (1971) 528

[25] J. Gregory, "Effects of polymers on colloid stability" in "The Scientific basis of flocculation", NATO advanced study institutes series, Sijthoff \& Noordhoff (1978) 101

[26] D. H. Napper, "Flocculation of sterically stabilized dispersions" J. Colloid Interface Sci., 32 (1970) 106

[27] D. H. Napper, “Steric stabilization” J. Colloid Interface Sci., 58 (1977) 390

[28] D. H. Napper, "Polymeric stabilization of colloidal dispersions" Academic Press, London (1983)

[29] D. W. J. Osmond, B. Vincent, F. A. Waite, "Steric stabilisation: A reappraisal of current theory" Colloid \& Polymer Sci. 253 (1975), 676

[30] M. Smoluchowski, "Versuch einer mathematischen Theorie der Koagulationskinetic kolloider Lösungen.“, Z. Phis. Chem, 92 (1917)129

[ 31] K. Higaschitani, Y. Matsuno, "Rapid Brownian coagulation of colloidal dispersions", J. Chem. Phys., 812 (1979) 460

[32] H. Sonntag, "Coagulation kinetics" in "Coagulation and flocculation, theory and applications” (ed. by B. Dobiás), Marcel Dekker, New York, (1993) 57 
[33] N. Fuchs "Über die Stabilität und Aufladung der Aerosole“, Z. Phys., 89 (1934) 736

[34] H. Reerink, J. Th. G. Overbeek, "The rate of coagulation as a measure of the stability of silver iodide sols.”, Discuss. Faraday Trans., 18 (1954) 74

[35] G. R. Zeichner, W. R. Schowalter, "Use of trajectory analysis to study stability of colloidal dispersions in flow fields" AIChE J., 23 (1977) 243

[36] H. S. Chung, R. Hogg, "Stability criteria for fine-particle dispersions) Colloid Surf. 15 (1985) 119.

[37] T. G. M van de Ven, S.G. Mason, "Microrheology of colloidal dispersions.VII. Orthokinetic doublet formation of spheres." Colloid Polymer Sci., 479 (1977) 255

[38] W. R. Schowalter, "Stability and coagulation of colloids in shear fields", Ann. Rev. Fluid Mech., 16 (1984) 245

[39] M. R. Greene, D. A. Hammer, and W. L. Olbricht, "The effect of hydrodynamic flow field on colloidal stability", J. Colloid Interface Sci. 167, (1994) 232

[40] B. K. Brunk, D. L. Koch, L. W. Lion, "Turbulent coagulation of colloidal particles”, J Fluid Mech., 364 (1998) 84

[41] B. K. Brunk, D. L. Koch, „Hydrodynamic pair diffusion in isotropic random velocity fields with application to turbulent coagulation“, Phys. Fluids, 9 (1997) 2670

[42] T. G. M van de Ven, S.G. Mason, "Microrheology of colloidal dispersions. IV. Pairs of Interacting Spheres in Shear Flow", J. Colloid Interface Sci., 57 (1976) 505

[43] G. R. Zeichner, W. R. Schowalter, "Effect of hydrodynamic and colloidal forces on the coagulation of dispersions“,J. Colloid Interface Sci., 71 (1979) 237

[44] P.M. Adler, "Interaction of unequal spheres, I. Hydrodynamic interactions: Colloidal forces", J. Colloid Interface Sci, 84 (1981) 461

[45] P.M. Adler, "Interactions of unequal spheres II. Conducting spheres" J. Colloid Interface Sci, 84 (1981) 475

[46] P.M. Adler, "Interactions of unequal spheres III. Experimental", J. Colloid Interface Sci, 84 (1981) 489

[47] P.M. Adler, "Heterocoagulation in shear flow" J. Colloid Interface Sci., 83 (1981) 106

[48] Q. Wang, “A study on shear coagulation and heterocaogulation", J. Colloid Interface Sci., 150 (1992) 418.

[49] A. K. Chesters, "The modelling of coalescence processes in fluid-liquid dispersions: a review of current understanding” Trans. I. Chem. E., Part I, 69, (1991) 259. 
[50] T. G. M van de Ven, S.G. Mason, "The Microrheology of colloidal dispersions. VIII. Effect of shear on perikinetic doublet formation”, Coll. Polym. Sci. 255 (1977) 794

[51] D. L. Feke, W. R. Schowalter, "The effect of shear-induced coagulation of colloid dispersions", J. Fluid Mech., 133 (1983) 17

[52] D. L. Feke, W. R. Schowalter, "The influence of Brownian diffusion on binary flow-induced collision rates in colloidal dispersions", J. Colloid Interface Sci., 106 (1986) 203

[53] S. Melis, M. Verduyn, G. Storti, J. Baldyga, M. Morbidelli, "Effect of fluid motion on aggregation of small particles subject to interaction forces", AIChE J., 45 (1999) 1383

[54] K. J. Ives, "The scientific basis of filtration" NATO Advanced Study Institute Series E: Applied science, vol.2. Noordhoff (1975)

[55] C. Tien, A. C. Payatakes, “Advances in deep bed filtration”, AIChE, 25 (1979) 735

[56] P. Bacchin, P. Aimir, V. Sanchez, "Model for colloidal fouling of membranes", AIChE J. 41 (1995) 368

[57] J. N. Ryan, M. Elimelech, "Colloid mobilization and transport in groundwater" Colloids Surf. A 107 (1996) 1

[58] A. L. Packman, J. S. MacKay," Interplay of stream-subsurface exchange, clay particle deposition, and streambed evolution”, Water Resour. Res., 39 (2003) 1097

[59] E. Boek, H. Ladva, J. Crawshaw, J. Padding, "Deposition of colloidal asphdltene in capillary flow: experiments and mesoscopic simulations" Energy \& Fuels, 22, (2008) 805

[60] T. Kulrattanarak, R. G. M. van der Sman, C. G. P. H. Schroen, R. M. Boom, "Classification and evaluation of microfluidic devices for continuous suspension fractionation” Adv. Colloid Interface Sci., 142 (2008) 53

[61] B. Mustin B. Stoeber, "Design considerations for robust suspension-based microfluidic systems for biomedical applications", "Proceedings of the 2008 IEEE 14th International Mixed-Signals, Sensors, and Systems Test Workshop, Vancouver, British Columbia, Canada, June 18-June 20

[62] B. Stoeber, E. Español, and D. Liepmann, "Operational Limits of Suspension Flow through Sudden Contractions," Proceedings of the 2001 ASME International Mechanical Engineering Congress and Exposition, New York, New York, U.S.A., November 11-16 2001, disc set vol. 2, MECE2001/MEMS-23878 
[63] J. Kao, Y. Wang, R. \& S. Weinbaum, "A theoretical model for nuclepore filters including hydrodynamic and molecular wall interaction effects“ J. Colloid Interface Sci. 125 (1988) 543

[64] H. M. Wyss, D. L. Blair, J. F. Morris, H. A. Stone, D. A. Weitz, "Mechanism for clogging of microchannels", Phys. Rev. E 74 (2006) 061402

[65] V. Ramachandran, H. S. Fogler, "Plugging by hydrodynamic bridging during flow of stable colloidal particles within cylindrical pores”, J. Fluid Mech., 385 (1999) 129

[66] C. W. Macosko, "Rheology: principles, measurements and applications", WileyVCH Publishers, Inc, 1994.

[67] E. G. M. Pelssers, M. A. Cohen Stuart, G. J. Fleer, "Single particle optical sizing (SPOS) I. Design of an improved SPOS instrument and application to stable dispersions", J. Colloid Interface Sci., 137 (1990) 350

[68] E. G. M. Pelssers, M. A. Cohen Stuart, G. J. Fleer, "Single particle optical sizing (SPOS) II. Hydrodynamic forces and application to aggregating dispersions", J. Colloid Interface Sci., 137 (1990) 362

[69] C. Turbitt, P. O’Hagan, K. Hasapidis, G. Pokrajac, “A multi-tool approach to colloid stability: SPOS and separation analysis”, J. Disp. Sci. Tech., 23 (2002) 711

[70] J. P. Bare, T. A. Lemke, "Monitoring slurry stability to reduce process variability", MICRO, 15 (1997) 53

[71] L: H. Hanus, S. A. Battafarano, A. R. Wank, "Optimizing the sizing and counting accuracy of CMP slurry large-particle counts”, MICRO, 21 (2003) 71

[72] T. Kuntzsch, U. Witnik, M. Hollatz, M. Stintz, S. Ripperger, "Characterisation of slurries used for chemical-mecahnical polishing (CMP) in the semiconductor industry“", Chem. Eng. Technol. 12 (2003) 1235

[73] E. E. Remsen et al., "Analysis of large particle count in fumed silica slurries and its correlation with scratch defects generated by CMP” J. Electrochem. Soc., 153 (2006) G453.

[74] “AccuSizer 780 User Manual“, Santa Barbara, CA: Particle Sizing Systems (2003)

[75] D. Dieterich, "Aqueous emulsions, dispersions and solutions of polyurethanes; synthesis and properties“ Prog. Org. Coat., 9 (1981) 281

[76] "PCS Software Help Manual“ Beckman Coulter, Inc. (2003) 
[77] J. C. Maxwell, "A treatise on electricity and magnetism, vol. 1", New York, Dover Publ, (1954)

[78] R. Barchini, D. A. Saville, "Dielectric response measurements on concentrated colloidal dispersions“,J. Colloid Interface Sci.,173 (1995) 86

[79] D. S. McLean, D. Vercoe, K. R. Stack, D. Richardson, "The colloidal pK $\mathrm{a}_{\mathrm{a}}$ of lipophilic extractives commonly found in Pinus radiate", Appiata J., 58 (2005) 362

[80] G. Fritz, W. Pechhold, N. Willenbacher, N. J. Wagner, "Characterising complex fluids with high frequency rheology using torsional resonators at multiple frequencies“, J. RHeol. 47 (2003) 303

[81] J. Bergenholtz, N. Willenbacher, N. J. Wagner, B. Morrison, D. van den Ende, J. Mellema, "Colloidal charge determination in concentrated liquid dispersions using torsional resonance oscillation“, J. Colloid Interface Sci., 202 (1998) 430

[82] R. A. Lionberger, W. B. Russel, "High frequency modulus of hard sphere colloids" J. Rheol., 38 (1994) 1885

[83] D. Quemada, "Rheology of concentrated disperse systems and minimum energy dissipation principle, I. viscosity -concentration relationship", Rheol. Acta, 16 (1977) 82

[84] P. N. Pusey, W. van Megen, "Phase behaviour of concentrated suspensions of nearly hard colloidal spheres", Nature 320 (1986) 340

[85] W. van Megen, S. M. Underwood, "Glass transition in colloidal hard spheres: Measurement and mode-coupling-theory analysis of the coherent intermediate scattering function", Phys. Rev. E, 49 (1994) 4206

[86] S. P. Meeker, W. C. K. Poon, P. N. Pusey, "Concentration dependence of the lowshear viscosity of suspensions of hard-sphere colloids" Phys. Rev. E, 55 (1997) 5718

[87] S.-E. Phan, W. B. Russel, Z. Cheng, J. Zhu, "Phase transition, equation of state, and limiting shear viscosities of hard sphere dispersions" Phys. Rev. E 54 (1996) 6633

[88] C. G. de Kruif, E. M: F. van Iersel, A. Vrij, W. B. Russel, "Hard sphere colloidal dispersions: Viscosity as a function of shear rate and volume fraction", J. Chem. Phys. 83 (1986) 4717

[89] L. Marshall, C. F. Zukoski, "Experimental studies on the rheology of hard-sphere suspensions near the glass transition“, J. Phys. Chem. 94 (1990) 1164 
[90] F. M. Horn, W. Richtering, "Viscosity of bimodal charge-stabilized polymer dispersions“, J. Rheol., 44 (2000) 1279

[91] R. Buscall, P. D’Haene, J. Mewis, "Maximum density for flow of dispersions of near monodisperse spherical-particles" Langnuir, 10 (1994) 1439

[92] R. Ranganathan, M Petric, R. Medina, U. Garcia, B.Bales, "Size, hydration and shape of sds/heptane micelles investigated by time- resolved fluorescence quenching and electron spin resonance" Langmuir, 17 (2001) 6765.

[93] R. J. Kuo, E. Matijevic, "Particle adhesion and removal in model systems Part 2.Monodispersed chromium hydroxide on steel” J. Chem. Soc. Faraday I., 75 (1979) 2014.

[94] J. Kao, Y. Wang, R. Pfeffer, S. Weinbaum, “A theoretical model for nuclepore filters including hydrodynamic and molecular wall interaction effects" J. Colloid Interface Sci. 121 (1988) 543

[95] A. Zaccone, H. Wu, M. Lattuada, M. Morbidelli, "Correlation between colloidal stability and surfactant adsorption/association phenomena studied by Light Scattering" J. Phys. Chem. B, 112 (2008) 1976.

[96] A. Zaccone, H. Wu, M. Lattuada, M. Morbidelli, "Charged molecular films on brownian particles: structure, interactions, and relation to stability", J. Phys. Chem. B, 112 (2008) 6793.

[97] H. Wu, A. Zaccone, A. Tsoutsoura, M. Lattuada, M. Morbidelli, "High shearinduced gelation of charge- stabilized colloids in a microchannel without adding electrolytes" Langmuir 25 (2009) 4715

[98] H. Wu, A. Tsoutsoura, M. Lattuada, A. Zaccone, M. Morbidelli, "Effect of temperature on high shear-induced gelation of charge-stabilized collids without adding electrolytes" Langmuir 26 (2010) 2761

[99] A. R. Studart, E. Amstad, L.J. Gauckler, "Colloidal stabilization of nanoparticles in concentrated suspensions", Langmuir, 23 (2007) 1081.

[100] K. Holmberg, B. Jönsson, B. Kronberg, B. Lindman, "Surfactants and Polymers in Aqueous Solution“, John Wiley \& Sons Ltd, West Sussex, 2003

[101] A. Zaccone, H. Wu, D. Gentili and M. Morbidelli, "Theory of activated-rate process under shear with application to shear-induced aggregation of colloids" Phis. Rev. E, 80 (2009) 051404

[102] D. Xie, H. Wu, A. Zaccone, L. Braun, H. Chen and M. Morbidelli, "Criticality for shear-induced gelation of charge-stabilized colloids", Soft Matter, 6 (2010) 2692 
[103] D. Xie, H. Wu, A. Zaccone, L. Braun, H. Chen and M. Morbidelli, "Criticality for shear-induced gelation of charge-stabilized colloids"-Supplementary Material (ESI), "Soft Matter, 6 (2010) 2692

[104] O. Molerus, M. H. Pahl, H. Rumpf, "Die Porositätsfunktion in empirischen gleichungen für durchströmungswiderstand im Bereich $\mathrm{Re} \leq 1$ “, Chemie-Ing.-Techn., 43 (1971) 376 


\section{Lebenslauf}

\section{Persönliche Daten}

Name Kristina Georgieva

Geburt 30.07.1981

Geburtsort Pomorie (Bulgarien)

Staatsangehörigkeit Bulgarien

Familienstand verheiratet

\section{Ausbildung}

$\begin{array}{ll}1988-1996 & \text { Grundschule, Nesebar (Bulgarien) } \\ 1996-2000 & \text { Fachschule für Wirtschaft, Burgas (Bulgarien) } \\ 2000-2004 & \begin{array}{l}\text { Bachelor-Studium, Fakultät für Chemie der Universität Sofia „St. } \\ \text { Kliment Ohridski“, Sofia (Bulgarien) }\end{array} \\ 2004-2006 & \begin{array}{l}\text { Master-Studium, Fakultät für Chemie, der Universität Sofia „St.- } \\ \text { Kliment-Ohridski“, Sofia (Bulgarien) }\end{array}\end{array}$

\section{Berufliche Tätigkeiten}

2006 - $2011 \quad$ Wissenschaftliche Mitarbeiterin am Institut für Mechanische Verfahrenstechnik und Mechanik des Karlsruher Instituts für Technologie (KIT)

seit 07/20011 Laborleiterin im Bereich „Advanced Material \& System Research“, BASF SE, Ludwigshafen 